\title{
Cobertura por circuitos em grafos mistos
}

\author{
Orlando Lee
}

\author{
TESE APRESENTADA \\ $\mathrm{AO}$ \\ INSTITUTO DE MATEMÁTICA E ESTATÍSTICA \\ DA \\ UNIVERSIDADE DE SÃO PAULO \\ PARA \\ OBTENÇÃO DO GRAU DE DOUTOR \\ EM \\ MATEMÁTICA APLICADA
}

Área de Concentração: Ciência da Computação

Orientadora: Profa. Dra. Yoshiko Wakabayashi

Durante a elaboração deste trabalho o autor recebeu apoio financeiro da CAPES

- São Paulo, novembro de 1999 - 


\title{
Coberturas por circuitos em grafos mistos
}

\author{
Este exemplar corresponde à redação \\ final da tese devidamente corrigida \\ e defendida por Orlando Lee \\ e aprovada pela comissão julgadora.
}

São Paulo, 17 de janeiro de 2000.

Banca examinadora:

- Prof. Dra. Yoshiko Wakabayashi (orientadora) (IME-USP)

- Prof. Dr. José Coelho de Pina Jr. (IME-USP)

- Prof. Dr. Ricardo Dahab (IC-UNICAMP)

- Prof. Dr. Jayme Luiz Szwarcfiter (NEC-UFRJ)

- Prof. Dr. Manoel José Machado Soares Lemos (UFPE) 


\section{Resumo}

Neste trabalho estudamos vários tipos de problemas envolvendo circuitos em grafos mistos. Tais grafos generalizam a noção de grafos orientados e não-orientados, no sentido de poderem conter tanto arcos como arestas.

O seguinte problema é tratado extensivamente em nosso trabalho: dado um grafo misto $M$ com pesos inteiros não-negativos $p(e)$ associados a cada arco/aresta $e$ de $M$, decidir se existe uma coleção de circuitos de $M$ tal que cada arco/aresta $e$ de $M$ pertence a exatamente $p(e)$ circuitos dessa coleção. Apresentamos uma boa caracterização para o problema assim como um algoritmo polinomial, baseado no método dos elipsóides, para o caso em que $M$ é um grafo misto série-paralelo. Além disso, mostramos que esse problema é NP-difícil para grafos mistos planares. Consideramos também uma relaxação linear desse problema e descrevemos resultados de polinomialidade/complexidade similares.

Resultados sobre dois problemas combinatórios bem conhecidos, o problema de detectar/encontrar circuitos negativos e o problema de encontrar caminhos mínimos, também são apresentados. Seu estudo foi motivado pelas implicações algorítmicas para os problemas mencionados acima. Mostramos que esses problemas são NP-difíceis para grafos mistos planares.

Estudamos também o problema de cobrir os arcos e as arestas de um grafo misto com circuitos de modo a minimizar a soma dos comprimentos dos circuitos. Discutimos vários resultados sobre a complexidade (de casos especiais) do problema, algoritmos de aproximação e sua relação com o problema do carteiro chinês. Descrevemos algoritmos polinomiais para o problema em grafos mistos com largura arbórea limitada.

Por fim, estudamos uma famosa conjectura de Woodall que relaciona circuitos e transversais em grafos orientados planares. Provamos que a conjectura é verdadeira para grafos orientados série-paralelos. 


\section{Agradecimentos}

À Yoshiko pela orientação, pelos bate-papos, pela leitura dos capítulos e pelas sugestões nesta reta final, e principalmente por ter me ensinado o gosto pela pesquisa.

A todos meus amigos e colegas do IME: Claus (e Fátima), Jair, Márcio, Alan, Ana Cláudia, Juaci, Lucia, Fábio, Scal, Fabiana, Elói, e Pablo, por terem ajudado a tornar essa fase da minha vida mais agradável.

Ao Flávio Keidi e ao Marko Loparic, pelo interesse constante.

À Marie e ao Bira, pela amizade e pela torcida.

Ao Mané e à Sônia, por tudo.

Ao Coelho e à Cris, pelos bate-papos e pelo interesse.

Aos professores do MAC e dos demais departamentos, que contribuíram para minha formação.

Aos funcionários do IME, pelo suporte na parte infra-estrutural e burocrática. 


\begin{abstract}
We study several problems on circuits in mixed graphs. These graphs generalise the notion of undirected and directed graphs, in the sense that they may contain edges as well as arcs.

The following problem is extensively studied in our work: given a mixed graph $M$ with non-negative integer weights $p(e)$ associated with each arc/edge $e$ of $M$, decide whether there exists a collection of circuits of $M$ such that each arc/edge $e$ of $M$ is contained in exactly $p(e)$ circuits from this collection. We give a good caracterization for the problem and describe a polynomial (ellipsoid based) algorithm for the case in which $M$ is a series-parallel mixed graph. Furthermore, we show that the problem is NP-hard for planar mixed graphs. We also consider a linear relaxation of this problem and describe similar results on complexity and polinomiality.

Results on two well-known combinatorial problems, the problem of detecting negative circuits and the problem of finding shortest paths, are also presented. This study was motivated by the algorithmic consequences for the problems above mentioned. We prove that these problems are NP-hard for planar mixed graphs.

We also study the problem of covering the arcs and the edges of a mixed graph with circuits so as to minimize the sum of the lenghts of the circuits. We discuss results on the complexity of (special cases of) this problem, approximation algorithms and its relationship with the Chinese Postman problem. We describe polynomial algorithms for the problem restricted to mixed graphs of bounded tree-width.

Finally, we study a famous conjecture of Woodall which relates circuits and transversals in planar directed graphs. We show that this conjecture holds for series-parallel directed graphs.
\end{abstract}




\section{Índice}

Introdução $\quad$ i

1 Preliminares 1

1.1 Teoria $\operatorname{dos}$ grafos $\ldots \ldots \ldots \ldots \ldots \ldots$

1.2 Poliedros e otimização . . . . . . . . . . . . . . . . 4

2 Cobertura por circuitos em grafos $\quad 7$

2.1 Introdução. . . . . . . . . . . . . . . . . 7

2.2 Definições e algumas observações iniciais . . . . . . . . . . . . . 8

$2.3 \mathrm{PCEC} / \mathrm{PCFC}$ em grafos orientados . . . . . . . . . . . . 8

$2.4 \mathrm{PCEC} / \mathrm{PCFC}$ em grafos não-orientados $\ldots \ldots \ldots \ldots$

2.5 PCEC/PCFC em grafos mistos . . . . . . . . . . . . . 16

2.6 Um algoritmo para o PCEC em um grafo misto série-paralelo . . . . . . 21

2.6.1 Circuitos mínimos em grafos mistos série-paralelos . . . . . . . 23

2.7 Cobertura uniforme por circuitos . . . . . . . . . . . . 25

2.8 Bases de Hilbert, subespaços lineares e reticulados . . . . . . . . . . 27

2.9. Conclusão . . . . . . . . . . . . . . . . . . . 32

3 Cobertura exata por circuitos em grafos mistos planares $\quad 35$

3.1 Introdução. . . . . . . . . . . . . . . . . . 35

3.2 Complexidade do PCEC/PCFC Planar . . . . . . . . . . . 35 
4 O problema do caminho mínimo e do circuito negativo 43

4.1 Introdução. . . . . . . . . . . . . . . . . . 43

$4.2 \quad \mathrm{PCN}$ versus PCFC . . . . . . . . . . . . . . . . . 44

4.3 A complexidade do PCN/PCM Planar . . . . . . . . . . . . . 45

5 O problema da cobertura mínima por circuitos 51

5.1 Introdução . . . . . . . . . . . . . . . . . 51

5.2 Complexidade do PCMC . . . . . . . . . . . . . . . . 52

5.3 Casos particulares do PCMC . . . . . . . . . . . . 56

5.4 Decomposição e largura arbórea . . . . . . . . . . . . . . . . . . 59

5.4 .1 O PCC em grafos com largura arbórea limitada . . . . . . . . . 63

5.4 .2 Descrição de um algoritmo para o PCC . . . . . . . . . . . 65

5.4.3 O PCMC em grafos com largura arbórea limitada . . . . . . . 67

5.4 .4 Descrição de um algoritmo para o PCMC . . . . . . . . . . . 69

5.4.5 Outros problemas em grafos de largura limitada . . . . . . . . 72

5.5 Algoritmos de aproximação para o PCMC . . . . . . . . . . . . . . . . 72

6 A conjectura de Woodall $\quad 78$

6.1 Introdução. . . . . . . . . . . . . . . . . 78

6.2 Definições e resultados conhecidos . . . . . . . . . . . . . 78

6.3 Prova da conjectura para grafos série-paralelos . . . . . . . . . . 80

7 Considerações finais $\quad 84$

Referências Bibliográficas $\quad 86$

Índice Remissivo $\quad 92$ 


\section{Lista de Figuras}

2.1 Grafo de Petersen. . . . . . . . . . . . . . . . . . . . 10

2.2 Grafo $M K_{4} \ldots \ldots \ldots \ldots \ldots \ldots \ldots \ldots \ldots \ldots$

3.1 (i) Grafo $G(F)$ da fórmula $F=(a+\bar{b}+c)(b+c+\bar{d})$.

(ii) Grafo $\vec{G}(F) . \ldots \ldots \ldots \ldots \ldots$

3.2 Grafo $L\left(v_{j}\right)$ correspondente à variável $v_{j} \ldots \ldots \ldots \ldots$. . . . . . 37

3.3 (i) Grafo $H\left(C_{i}\right)$ correspondente a uma cláusula $C_{i}$ contendo 3 literais.

(ii) Grafo $H\left(C_{i}\right)$ correspondente a uma cláusula $C_{i}$ contendo 2 literais. . . 38

3.4 Grafo $(M, p)$ obtido da fórmula $F=(a+\bar{b}+c)(b+c+\bar{d}) \ldots \ldots . \ldots 39$

3.5 Caminho em ziguezague. . . . . . . . . . . . . . . . 40

3.6 Possíveis circuitos na cobertura de $H\left(C_{i}\right) \ldots \ldots \ldots \ldots$. . . . . . 40

4.1 Grafo $L\left(v_{j}\right)$ correspondente à variável $v_{j} \ldots \ldots \ldots \ldots$. . . . . . 45

4.2 (i) Grafo $H\left(C_{i}\right)$ correspondente a uma cláusula $C_{i}$ contendo 3 literais.

(ii) Grafo $H\left(C_{i}\right)$ correspondente a uma cláusula $C_{i}$ contendo 2 literais. . . 46

4.3 Grafo $(M, c)$ obtido da fórmula $F=(a+\bar{b}+c)(b+c+\bar{d}) \ldots \ldots \ldots 4$

4.4 Caminho em ziguezague. . . . . . . . . . . . . . . . . 48

4.5 Possíveis circuitos de peso 1 em $H\left(C_{i}\right) . \ldots \ldots \ldots \ldots$. . . . . . 48



5.2 Uma decomposição arbórea $\left(T,\left\{W_{1}, \ldots, W_{10}\right\}\right)$ do grafo $G . \ldots \ldots 1$

6.1 Contra-exemplo de Schrijver. Os arcos em negrito têm peso 1 enquanto os demais têm peso $0 \ldots \ldots \ldots 79$ 


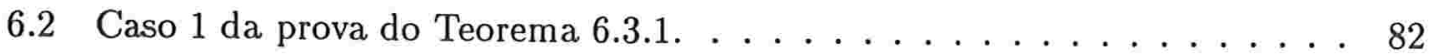

6.3 Caso 2 da prova do Teorema 6.3.1. . . . . . . . . . . 83 


\section{Lista de Tabelas}

2.1 Tabela dos principais resultados sobre o PCEC. . . . . . . . . . . . . 33

2.2 Tabela dos principais resultados sobre o PCFC. . . . . . . . . . . . . 34

4.1 Tabela dos principais resultados sobre o PCN. . . . . . . . . . . . 50

4.2 Tabela dos principais resultados sobre o PCN . . . . . . . . . . 50

5.1 Tabela dos principais resultados sobre o PCEC . . . . . . . . . . 77

7.1 Tabela com os resultados sobre o PCEC, o PCFC, o PCN, o PCM e o

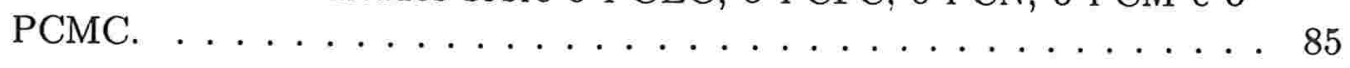




\section{Introdução}

O problema de decidir se um grafo não-orientado admite um passeio fechado que passa exatamente uma vez por todas suas arestas é considerado um dos problemas mais antigos da teoria dos grafos. Pode-se dizer que sua origem remonta a 1736, quando Leonhard Euler resolveu o famoso problema das sete pontes de Königsberg [29]. Para grafos conexos este problema é equivalente ao de decidir se um grafo pode ser decomposto em um conjunto de circuitos disjuntos nas arestas. Não é difícil ver que um grafo (não necessariamente conexo) admite uma tal decomposição se, e somente se, cada vértice tem grau par. Para grafos orientados temos uma caracterização análoga: um grafo orientado admite uma decomposição em circuitos orientados disjuntos nos arcos se, e somente se, em cada vértice $v$ o número de arcos que entram em $v$ é igual ao número de arcos que saem de $v$. Se considerarmos grafos mistos, isto é, grafos que possuem tanto arestas quanto arcos, o problema torna-se um pouco mais complicado, mas para tais grafos também existe uma boa caracterização (entendendo-se neste caso que circuitos possuem todos os seus arcos direcionados no mesmo sentido).

Neste trabalho estudamos algumas generalizações e problemas correlatos ao de decidir se um grafo misto admite uma decomposição em circuitos disjuntos. Um grafo misto $M$ é definido formalmente como uma tripla $M:=(V, E, A)$ onde $V$ é um conjunto de vértices, $E$ é um conjunto de arestas e $A$ é um conjunto de arcos. Assim, grafos orientados e nãoorientados podem ser vistos como classes especiais de grafos mistos.

O primeiro problema que consideramos é o Problema da Cobertura Exata por Circui$\operatorname{tos}^{1}$ (PCEC): dado um par $(M, p)$ onde $M$ é um grafo misto e $p$ é uma função peso que associa a cada arco/aresta $e$ de $M$ um inteiro não-negativo $p(e)$, decidir se este admite uma cobertura exata por circuitos. Mais precisamente, decidir se existe uma coleção de circuitos de $M$ tal que cada arco/aresta $e$ de $M$ pertence a exatamente $p(e)$ circuitos dessa coleção. Claramente, os problemas mencionados no primeiro parágrafo são casos especiais do PCEC.

\footnotetext{
${ }^{1}$ Este problema é conhecido na literatura como circuit cover problem, razão pela qual optamos por esta nomenclatura, embora um nome mais natural talvez fosse Problema da Decomposição em Circuitos.
} 
Uma formulação mais algébrica e equivalente do PCEC é a seguinte: dado um grafo misto $M=(V, E, A)$ e $p \in \mathbb{Z}_{+}^{E \cup A}$, decidir se existem inteiros não-negativos $\lambda_{1}, \ldots, \lambda_{t}$ e circuitos $C_{1}, \ldots, C_{t}$ de $M$ tais que

$$
p=\sum_{i=1}^{t} \lambda_{i} \chi^{C_{i}},
$$

onde $\chi^{F} \in\{0,1\}^{E \cup A}$ denota o vetor de incidência de um conjunto $F \subseteq E \cup A$, isto é, $\chi^{F}(e)=1$ se $e \in F$, e $\chi^{F}(e)=0$ caso contrário. Podemos considerar uma relaxação natural do PCEC permitindo que $\lambda_{1}, \ldots, \lambda_{t}$ sejam racionais não-negativos em vez de inteiros não-negativos. Chamamos essa versão relaxada de Problema da Cobertura Fracionária por Circuitos (PCFC).

No Capítulo 1 introduzimos a notação e as definições que são utilizadas ao longo do trabalho.

No Capítulo 2 estudamos o PCEC e o PCFC. Os primeiros resultados conhecidos envolvendo esses problemas foram obtidos por Hoffman [42] em 1960. Ele mostrou que quando $M$ é um grafo orientado, ambos os problemas são basicamente equivalentes e podem ser resolvidos em tempo polinomial. O caso em que $M$ é um grafo não-orientado tem sido o mais estudado na literatura. Em 1979 Seymour [65] mostrou uma boa caracterização para o PCFC em grafos não-orientados arbitrários e também para o PCEC em grafos não-orientados planares. Apesar disto, o PCEC não foi totalmente resolvido; como veremos, ainda restam várias questões não respondidas. O PCEC está estreitamente relacionado com vários problemas famosos: a conjectura da 3-aresta-coloração de Tutte, a conjectura da cobertura dupla de circuitos e o Problema do Carteiro Chinês. O resultado mais importante sobre o PCEC, obtido em 1994 por Alspach, Goddyn e Zhang [2], consiste em uma boa caracterização para o PCEC restrito a grafos não-orientados que não contêm um menor do grafo de Petersen, estendendo o resultado anterior de Seymour para grafos planares. Entretanto, veremos que mesmo com essa caracterização não se conhece nenhum algoritmo polinomial para o correspondente problema de busca, isto é, o problema de encontrar, se existir, uma cobertura exata por circuitos. Para grafos mistos, ambos os problemas foram bem menos estudados. Em 1986, Arkin e Papadimitriou $[10,8]$ mostraram que o PCFC e PCEC para grafos mistos são NP-completos. Mostramos neste capítulo uma boa caracterização para cada um desses problemas no caso em que $M$ é um grafo misto série-paralelo. Para este caso também descrevemos um algoritmo polinomial baseado no método dos elipsóides para suas versões de busca. Seguindo em outra direção, apresentamos alguns resultados sobre bases de Hilbert, subespaços lineares e reticulados associados aos (vetores de incidência dos) circuitos de um grafo misto.

Continuamos nosso estudo desses problemas no Capítulo 3, onde mostramos que o PCEC e o PCFC são NP-completos para grafos mistos planares. Esses resultados eram de grande interesse para nós, pois um dos nossos objetivos era ou estender nossos resultados sobre boa caracterização e polinomialidade para grafos mistos série-paralelos, ou mostrar que esses problemas restrito a grafos planares são NP-completos. 
No Capítulo 4 estudamos o Problema do Circuito Negativo (PCN): dado grafo misto $M$ com pesos associados a cada arco/aresta de $M$, decidir se existe um circuito de peso negativo. Trata-se de um problema clássico, interessante por si mesmo, mas seu estudo neste contexto foi motivado pelas suas implicações algorítmicas para o PCFC.

Sabe-se que o PCN para grafos mistos arbitrários é NP-completo [9, 8]. Mostramos neste capítulo que o PCN é NP-completo mesmo para grafos mistos planares. Também mostramos que o Problema do Caminho de Peso Mínimo para grafos mistos planares é NP-difícil.

No Capítulo 5 consideramos um problema de otimização envolvendo cobertura por circuitos, o Problema da Cobertura Mínima por Circuitos (PCMC): dado um grafo misto $M=(V, E, A)$, encontrar uma família $C_{1}, \ldots, C_{t}$ de circuitos de $M$ que cobre todos os arcos e arestas de $M$, isto é, cada arco/aresta $e$ pertence a pelo menos um circuito dessa família, e tal que o seu peso total $\sum_{i=1}^{t}\left|C_{i}\right|$ seja o menor possível. Inicialmente, apresentamos uma prova recente de Thomassen [70] de que o PCMC para grafos não-orientados arbitrários é NP-difícil. Este resultado implica imediatamente que o PCMC é NP-difícil para grafos mistos arbitrários. Em seguida apresentamos alguns casos particulares do PCMC, encontrados na literatura, para os quais se conhecem algoritmos polinomiais para resolvê-lo ou para os quais há evidências de que eles existem. Veremos que em todos esses casos existe uma equivalência entre o PCMC e o Problema do Carteiro Chinês (PCC). Nossa contribuição neste contexto foi estudar o PCMC restrito a grafos com um certo parâmetro, a largura arbórea, limitado por uma constante. Mostramos que para grafos mistos com largura arbórea limitada (definida como a largura do grafo subjacente) o PCC e o PCMC podem ser resolvidos em tempo polinomial (lembramos que o PCC é NP-difícil para grafos mistos planares e que grafos série-paralelos têm largura arbórea no máximo 2). Finalmente, consideramos algoritmos de aproximação para o PCMC e apresentamos uma demonstração de um resultado de Bermond, Jackson e Jaeger [15] (e Alon e Tarsi [7]) que diz que todo grafo não-orientado 2-aresta-conexo $G$ possui uma cobertura por circuitos de peso no máximo $5 / 3|E(G)|$.

O Capítulo 6 não está relacionado diretamente com os capítulos anteriores, embora trate de circuitos em grafos série-paralelos. Lá estudamos a conjectura de Woodall que afirma que em um grafo orientado planar, o comprimento de um circuito mínimo é igual à cardinalidade de uma coleção máxima de transversais disjuntas (uma transversal é um conjunto de arcos cuja remoção destrói todos os circuitos orientados). Mostramos neste capítulo que para grafos orientados série-paralelos essa conjectura é verdadeira. A demonstração deste resultado foi parcialmente inspirada no algoritmo descrito no Capítulo 2 para encontrar um circuito de peso mínimo em um grafo série-paralelo. 


\section{Preliminares}

Neste capítulo introduzimos alguns conceitos e estabelecemos a notação que utilizamos nos próximos capítulos.

Não incluímos nenhuma seção sobre teoria de complexidade computacional já que os conceitos que usaremos são bem conhecidos e também pelo fato de que adotaremos aqui a terminologia amplamente aceita de Garey e Johnson [37].

\subsection{Teoria dos grafos}

Apresentamos aqui alguns conceitos básicos da teoria dos grafos, com o propósito de estabelecer a terminologia e a notação que utilizamos neste trabalho. Não é nosso objetivo fazer uma abordagem abrangente, mas nos concentrarmos principalmente nas definições envolvendo grafos mistos que aparecem na maior parte deste trabalho. Para conceitos e definições relativos a grafos orientados e não-orientados seguimos basicamente a terminologia de Bondy e Murty [20].

Embora grafos mistos sejam generalizações naturais de grafos orientados e nãoorientados, não se conhecem muitos resultados sobre eles na literatura. O leitor interessado pode encontrar em [51] uma coletânea de vários problemas conhecidos envolvendo tais grafos. No entanto, a maioria dos problemas que tratamos neste trabalho não é mencionada nessa referência.

Um grafo misto é uma tripla $M=(V, E, A)$, onde $V$ é um conjunto de elementos chamados vértices, $E$ é um conjunto de elementos chamados arestas, e $A$ é um conjunto de elementos chamados arcos. A cada aresta $e$ de $M$ está associado um par não-ordenado $\{x, y\}$ de vértices, e a cada arco $a$ de $M$ está associado um par ordenado $(u, v)$; dizemos que os vértices $x, y$ (respectivamente, $u, v$ ) são os extremos de $e$ (respectivamente, $a$ ).

Usamos a notação $e=x y$ para indicar que $e$ é uma aresta de $M$ associada a $\{x, y\}$ e a notação $a=(u, v)$ para indicar que $a$ é um arco de $M$ associado a $(u, v)$. Por simplicidade, quando não há risco de ambigüidade dizemos simplesmente que $x y$ é uma 
aresta ou que $(u, v)$ é um arco de $M$.

Duas arestas distintas $e=x y, f=x y$ são chamadas arestas paralelas, e dois arcos $a=(u, v), b=(u, v)$ são chamados arcos paralelos. Dois arcos da forma $a=(u, v) \mathrm{e}$ $b=(v, u)$ são chamados arcos reversos. Um laço é uma aresta (um arco) da forma $v v$ $((v, v))$.

Às vezes nos referimos a um grafo misto $M$ sem especificar seus conjuntos de vértices, arestas e arcos; nesse caso, tais conjuntos são indicados respectivamente por $V(M), E(M)$ e $A(M)$.

Seja $M=(V, E, A)$ um grafo misto. Se $A=\emptyset$ dizemos que $M$ é um grafo nãoorientado e o indicamos simplesmente por $M=(V, E)$. Se $E=\emptyset$ dizemos que $M$ é um grafo orientado e o indicamos simplesmente por $M=(V, A)$. Às vezes, utilizamos a palavra grafo significando tanto um grafo misto quanto um grafo não-orientado. Neste trabalho reservamos as letras $M, G, D$ para indicar grafos mistos, grafos não-orientados e grafos orientados, respectivamente.

Às vezes, é conveniente considerar um grafo misto $M=(V, E, A)$ como uma união de um grafo orientado $D=(V, A)$ e um grafo não-orientado $G=(V, E)$. Dizemos então que $M$ é composto por $D$ e por $G$ e indicamos isto através da notação $M=D \sqcup G$. Nesse caso, dizemos também que $D$ é a parte orientada de $M$ e $G$ é a parte não-orientada de $M$. O grafo subjacente $G(M)$ de um grafo misto $M$ é o grafo não-orientado obtido de $M$ substituindo cada arco por uma aresta com os mesmos extremos.

Seja $G=(V, E)$ um grafo não-orientado. Dados subconjuntos $X, Y$ de $V$ definimos:

$$
\begin{aligned}
\delta_{G}(X, Y) & =\{x y \in E \mid x \in X, y \in Y\}, \\
\delta_{G}(X) & =\delta_{G}(X, V-X) .
\end{aligned}
$$

Seja $D=(V, A)$ um grafo orientado. Para $X, Y \subseteq V$ definimos:

$$
\begin{aligned}
& \delta_{D}^{-}(X)=\{(y, x) \in A \mid x \in X, y \in V-X\}, \\
& \delta_{D}^{+}(X)=\delta_{D}^{-}(V-X) .
\end{aligned}
$$

Nas definições que se seguem vamos supor que $M=(V, E, A)$ é um grafo misto composto pelo grafo orientado $D$ e pelo grafo não-orientado $G$.

Para $X \subseteq V$ definimos:

$$
\nabla_{M}(X)=\delta_{G}(X) \cup \delta_{D}^{-}(X) \cup \delta_{D}^{+}(X) .
$$

Para simplificar a notação, sempre que os grafos $M, D$ ou $G$ estão subentendidos dentro do contexto, estes são omitidos nas notações em que ocorrem como subscrito. Por exemplo, escrevemos $\nabla(X)$ em lugar de $\nabla_{M}(X)$. Outra simplificação que adotamos é a de que algumas vezes não distinguimos um conjunto formado por um único elemento do próprio elemento. Por exemplo, escrevemos $\nabla(v)$ e $X-x$ em vez de $\nabla(\{v\})$ e $X-\{x\}$.

Um subconjunto $B \neq \emptyset$ de arcos e arestas é um corte se existe $X \subseteq V$ tal que $F=\nabla(X)$. Dizemos que uma aresta $e$ é uma ponte ou aresta de corte se existe um corte formado unicamente por $e$. Um corte $F=\nabla(X)$ é um dicorte se $\nabla(X)=\delta^{+}(X)$. 
Um passeio $P$ (em $M$ ) é uma seqüência de vértices e arcos/arestas de $M$ da forma $P=\left(v_{1}, l_{1}, v_{2}, l_{2}, \ldots, v_{n-1}, l_{n-1}, v_{n}\right)$ onde $l_{i}=v_{i} v_{i+1}$ é uma aresta ou $l_{i}=\left(v_{i}, v_{i+1}\right)$ é um arco, para $1 \leq i \leq n-1$. Dizemos que $v_{1}$ é o início e $v_{n}$ é o final de $P$, e que $P$ é um passeio de $v_{1}$ a $v_{n}$. O comprimento de $P$, denotado por $|P|$, é definido como $n-1$.

Seja $P=\left(v_{1}, l_{1}, v_{2}, \ldots, v_{n-1}, l_{n-1}, v_{n}\right)$ um passeio em $M$. Dizemos que $P$ é um passeio fechado em $M$ se $|P| \geq 1$ e o seu início e o seu final coincidem. Se além disso, tivermos $v_{i} \neq v_{j}$ para $1 \leq i<j \leq n-1$, então um tal passeio fechado é chamado de circuito. Um caminho $P=\left(v_{1}, l_{1}, \ldots, l_{n-1}, v_{n}\right)$ em $M$ é um passeio em $M$ tal que $v_{i} \neq v_{j}$ para $1 \leq i<j \leq n$. Dizemos que $v_{2}, v_{3}, \ldots, v_{n-1}$ são os vértices internos e que $v_{1}$ e $v_{n}$ são os extremos do caminho $P$. Algumas vezes representamos um caminho ou um circuito apenas por sua seqüência de vértices $\left(v_{1}, \ldots, v_{n}\right)$; ou então não distinguimos o caminho (ou o circuito) em si do seu conjunto de arcos e arestas. Essas representações são usadas de acordo com nossa conveniência, sempre evitando ambigüidades.

Dado um grafo misto $M=(V, E, A)$ e $F \subseteq E \cup A$ denotamos por $M-F$ o grafo $(V, E-F, A-F)$ obtido de $M$ pela remoção de $F$. Dado um grafo misto $M=(V, E, A)$ e $e \in E \cup A$, definimos o grafo $M / e$ como o grafo obtido de $M$ removendo $e$ e identificando seus extremos. Dizemos que $M / e$ é o grafo obtido de $M$ pela contração de $e$. De um modo mais geral, se $F \subseteq E \cup A$ então denotamos por $M / F$ o grafo obtido de $M$ pela contração de $F$; mais precisamente, $M / F$ é obtido a partir de $M$ contraindo um a um, em qualquer ordem, os elementos de $F$. Dada uma aresta $e=x y$ de um grafo $M$, a subdivisão de $e$ em $M$ consiste em substituir a aresta $e$ por duas arestas $x z$ e $z y$ onde $z$ é um novo vértice.

Dados grafos não-orientados $G$ e $H$, dizemos que $H$ é um menor de $G$ se existe um subgrafo $G^{\prime}$ de $G$ e $F \subseteq E\left(G^{\prime}\right)$ tal que $G^{\prime} / F$ é isomorfo a $H$. Dizemos nesse caso que $G$ contém um menor de $H$. Dizemos que um grafo $G$ é livre de $H$ se não contém um menor de $H$. Dizemos que $H$ é uma subdivisão de um grafo $G$ se $H$ pode ser obtido a partir de $G$ através de uma seqüência de subdivisões de arestas. Se $H$ é um grafo cúbico então $G$ contém um menor de $H$ se, e somente se, $G$ contém uma subdivisão de $H$ como um subgrafo. Dado um grafo não-orientado $G$ denotamos por $\widetilde{G}$ o grafo (a menos de isomorfismo) obtido a partir de $G$ substituindo cada caminho maximal $P$ em $G$ no qual todos seus vértices internos têm grau dois por uma aresta ligando os extremos de $P$.

Um grafo não-orientado é série-paralelo se pode ser obtido a partir de uma árvore através de uma seqüência de subdivisões e duplicações de arestas. É um fato bem conhecido que $G$ é série-paralelo se e somente se não contém um menor do $K_{4}$. Dizemos que um grafo misto $M$ é série-paralelo se seu grafo subjacente $G(M)$ é série-paralelo.

Um grafo misto $M$ é fortemente conexo se para todo par ordenado $u, v$ de vértices de $V(M)$ existe um caminho de $u$ a $v$ em $M$. Um componente fortemente conexo é um subgrafo fortemente conexo e maximal de $M$.

Um grafo misto $M$ é euleriano se $|\delta(X)|-|| \delta^{-}(X)|-| \delta^{+}(X)||$ é não-negativo e par para todo $X \subseteq V(M)$. O seguinte teorema [14,35] generaliza os resultados bem conhecidos sobre os casos orientado e não-orientado. 
Teorema 1.1.1. Todo grafo misto euleriano pode ser decomposto em uma coleção de circuitos disjuntos nos arcos e nas arestas.

Nos casos particulares em que $M$ é um grafo não-orientado, respectivamente orientado, chamamos um subgrafo euleriano de $M$ de ciclo, respectivamente ciclo orientado. Como no caso de circuitos, algumas vezes não distinguimos um subgrafo euleriano do seu conjunto de arcos e arestas.

\subsection{Poliedros e otimização}

Nesta seção discutimos brevemente um resultado envolvendo problemas de otimização sobre poliedros que será necessário neste trabalho. Vamos assumir uma certa familiaridade com o assunto e nos concentrarmos nos aspectos que nos interessam. O leitor menos familiarizado com alguns desses tópicos pode consultar o livro de Grötschel, Lovász e Schrijver [39].

Para descrever os resultados precisamos introduzir algumas definições de natureza técnica. Dado um inteiro $n \neq 0$, definimos o tamanho (da codificação) de $n$ como

$$
\langle n\rangle:=1+\left\lceil\log _{2}(|n|+1)\right\rceil
$$

ou seja, $\langle n\rangle$ é o número de bits necessário para codificar $n$ na forma binária. De forma natural, o tamanho (da codificação) de um número racional $r=p / q$, com $p$ e $q$ relativamente primos, é definido como

$$
\langle r\rangle:=\langle p\rangle+\langle q\rangle
$$

e se $x$ é um vetor (ou uma matriz) racional então o tamanho (da codificação) $\langle x\rangle$ é definido como a soma dos tamanhos dos seus componentes. Finalmente, se $a^{T} x \leq \beta$ é uma desigualdade com $a \in \mathbb{Q}^{n}$ e $\beta \in \mathbb{Q}$ então o tamanho (da codificação) da desigualdade é definida como $\langle a\rangle+\langle\beta\rangle$.

Em combinatória poliédrica, é comum pensar em um subconjunto $F$ de arestas/arcos de um grafo $M=(V, E, A)$ como um vetor de incidência $\chi^{F}$ pertencente ao espaço $\mathbb{R}^{E \cup A}$, o conjunto dos vetores reais indexados por $E \cup A$. Mais precisamente, se $F \subseteq E \cup A$ então $\chi^{F} \in\{0,1\}^{E \cup A}$ é definido por $\chi^{F}(e)=1$, se $e \in F$, e $\chi^{F}(e)=0$, caso contrário. Isso permite formular vários problemas combinatórios como problemas de otimização em poliedros.

Um subconjunto $P \subseteq \mathbb{R}^{n}$ é um poliedro se é da forma $P=\left\{x \in \mathbb{R}^{n} \mid A x \leq b\right\}$, onde $A$ é uma matriz e $b$ é um vetor com dimensões apropriadas. Se é possível escolher $b=\mathbf{0}$ então dizemos que $P$ é um cone. Em nosso trabalho, consideramos apenas poliedros (e cones) racionais, ou seja, aqueles que podem ser descritos como acima por uma matriz racional $A$ e um vetor racional $b$.

Definimos o cone recessional de um poliedro $P$ como

$$
\operatorname{rec}(P):=\left\{z \in \mathbb{R}^{n} \mid x+\lambda z \in P \text { para todo } x \in P \text { e todo } \lambda \geq 0\right\} .
$$


Dado um poliedro $P \subseteq \mathbb{R}^{n}$ e um inteiro positivo $\varphi$, dizemos que $P$ tem complexidade de faceta no máximo $\varphi$ se existe um sistema de desigualdades $A x \leq b$ com coeficientes racionais tal que $P=\left\{x \in \mathbb{R}^{n} \mid A x \leq b\right\}$, e o tamanho da codificação de cada desigualdade é no máximo $\varphi$. Um poliedro bem-descrito é uma tripla $(P ; n, \varphi)$ onde $P \subseteq \mathbb{R}^{n}$ é um poliedro com complexidade de faceta no máximo $\varphi$. Definimos o tamanho da codificação $\langle P\rangle$ de um poliedro bem-descrito $(P ; n, \varphi) \operatorname{como} \varphi+n$.

Considere os dois seguintes problemas.

Problema de Otimização para Poliedros (POP). Dado um poliedro $P \subseteq \mathbb{R}^{n}$ e um vetor $c \in \mathbb{Q}^{n}$, ou

(i) certifique que $P$ é vazio, ou

(ii) encontre um vetor $x^{*} \in P$ que maximiza $c^{T} x$ em $P$, ou

(iii) encontre um vetor $z \in \operatorname{rec}(P)$ tal que $c^{T} z \geq 1$.

Problema da Separação para Poliedros (PSP). Dado um poliedro $P \subseteq \mathbb{R}^{n}$ e um vetor $y \in \mathbb{R}^{n}$, ou

(i) certifique que $y \in P$, ou

(ii) encontre um vetor $c \in \mathbb{R}^{n}$ tal que $c^{T} y>\max \left\{c^{T} x \mid x \in P\right\}$.

Note que na definição de POP e PSP não foi especificado como é codificado o poliedro $P$. Se $P$ é dado explicitamente, isto é, são dados $A$ e $b$ tais que $P=\left\{x \in \mathbb{R}^{n} \mid A x \leq b\right\}$, então o PSP é trivial, e o POP pode ser resolvido em tempo polinomial usando o método dos elipsóides, como foi mostrado por Khachiyan [48]. O POP torna-se mais interessante quando o poliedro $P$ não é dado explicitamente, mas em vez disso, é especificado por um oráculo de separação, isto é, um algoritmo que resolve o problema da separação para o poliedro $P$. Grötschel, Lovász e Schrijver [39] mostraram que é possível estender o método dos elipsóides para resolver o POP nesse caso.

Teorema 1.2.1 (Grötschel, Lovász e Schrijver). Existem algoritmos (baseados no método dos elipsóides) que, para todo poliedro bem-descrito $(P ; n, \varphi)$ especificado por um oráculo de separação, e para todo vetor $c \in \mathbb{Q}^{n}$,

1. resolve o problema de otimização $\max \left\{c^{T} x \mid x \in P\right\}$,

2. encontra um solução ótima básica de $\max \left\{c^{T} x \mid x \in P\right\}$, se este existir, $e$

3. encontra uma solução básica do problema dual, se este existir.

O número de chamadas ao oráculo de separação, e o número de operações aritméticas elementares executados pelos algoritmos são limitados por um polinômio em $\varphi$. 
Como um exemplo de aplicação do Teorema 1.2.1 vejamos um poliedro que aparece em outras partes do trabalho. Definimos a matriz de incidência dos circuitos de um grafo misto $M$ como a matriz com linhas indexadas pelos arcos/arestas de $M$ cujas colunas correspondem aos vetores de incidência de todos os circuitos de $M$. Suponha que $G$ seja um grafo não-orientado e seja $N$ a matriz de incidência dos circuitos de $G$. Seja $P:=\left\{x \in \mathbb{R}^{E} \mid N^{T} x \geq 0\right\}$. Então $(P ; m, m)$ é um poliedro bem-descrito, e o problema da separação para o par $P, y$ consiste em decidir se existe um circuito negativo no grafo $(G, y)$. Como este problema pode ser resolvido em tempo polinomial, pelo Teorema 1.2.1, concluímos que o problema de otimização sobre $P$ pode ser resolvido em tempo polinomial. 


\section{Cobertura por circuitos em grafos}

\subsection{Introdução}

Neste capítulo estudamos os dois seguintes problemas.

Problema da Cobertura Exata por Circuitos (PCEC)

Dado um grafo misto $M=(V, E, A)$ e uma função peso $p: E \cup A \mapsto \mathbb{Z}_{+}$, decidir se existe uma coleção de circuitos em $M$ tal que cada arco/aresta $e$ de $M$ pertence a exatamente $p(e)$ circuitos desta coleção.

\section{Problema da Cobertura Fracionária por Circuitos (PCFC)}

Dado um grafo misto $M=(V, E, A)$ e uma função peso $p: E \cup A \mapsto \mathbb{Q}_{+}$, decidir se existem racionais não-negativos $\lambda_{1}, \ldots, \lambda_{t}$ e circuitos em $M_{t} C_{1}, \ldots, C_{t}$ tais que $p(e)=\sum\left(\lambda_{i}: e \in C_{i}\right)$, para cada arco/aresta $e$ de $M$.

O PCEC pode ser visto como uma generalização do problema de decidir se um grafo é euleriano (o caso em que $p \equiv 1$ ), enquanto o PCFC é uma relaxação fracionária do primeiro. Embora os problemas acima estejam formulados como problemas de decisão estamos também interessados em algoritmos polinomiais para os correspondentes problemas de busca (isto é, encontrar tais coberturas). Veremos que em alguns casos, mesmo conhecendo-se uma boa caracterização para o problema de decisão, não é inteiramente trivial conseguir um algoritmo polinomial para o correspondente problema de busca.

Nas seções 2.3 e 2.4 apresentamos os principais resultados conhecidos e os problemas em aberto envolvendo o PCEC e o PCFC em grafos orientados e não-orientados, respectivamente.

Os primeiros resultados envolvendo esses problemas foram obtidos no caso em que $M=D$ é um grafo orientado. Neste caso, ambos os problemas podem ser resolvidos em tempo polinomial e são essencialmente equivalentes como veremos.

O caso no qual $M=G$ é um grafo não-orientado tem sido o mais estudado na 
literatura. O PCFC pode ser resolvido em tempo polinomial para grafos não-orientados arbitrários. O PCEC, por outro lado, não está totalmente resolvido: várias questões permanecem ainda sem resposta. Como veremos, o PCEC para grafos não-orientados está estreitamente relacionado com alguns problemas famosos: a conjectura de 3-arestacoloração de Tutte, a conjectura da cobertura dupla por circuitos e o problema do carteiro chinês.

Para grafos mistos, ambos os problemas foram bem menos estudados, talvez devido ao fato de Arkin e Papadimitriou [10, 8] terem provado que o PCEC e o PCFC são problemas NP-completos. Entretanto, na Seção 2.5 mostramos que para grafos mistos série-paralelos, há boas caracterizações para a existência de soluções do PCEC/PCFC e um algoritmo polinomial baseado no método dos elipsóides para encontrá-las.

\subsection{Definições e algumas observações iniciais}

Dados um grafo misto $M=(V, E, A)$ e $p: E \cup A \mapsto \mathbb{Z}_{+}$, dizemos que uma coleção $\left\{C_{1}, \ldots, C_{k}\right\}$ de circuitos (possivelmente com repetições) é uma cobertura exata por circuitos de $(M, p)$ se cada elemento $e \in E \cup A$ pertence a exatamente $p(e)$ desses circuitos. Quando tal coleção existir, dizemos que $(M, p)$ admite uma cobertura por circuitos. Assim, o PCEC consiste em decidir se $(M, p)$ admite uma cobertura exata por circuitos.

Uma forma mais compacta de representar uma cobertura exata é a seguinte. Dizemos que $(M, p)$ admite uma cobertura exata por circuitos se existem inteiros não-negativos $\mu_{1}, \ldots, \mu_{t}$ e circuitos distintos $C_{1}, \ldots, C_{t}$ tais que $p(e)=\sum\left(\mu_{i} \mid e \in C_{i}\right)$, para cada arco/aresta $e$ de $M$. Esta representação é utilizada na Seção 2.5.

Dados um grafo misto $M=(V, E, A)$ e $p: E \cup A \mapsto \mathbb{Z}_{+}$, dizemos que $(M, p)$ admite uma cobertura fracionária por circuitos se existem racionais não-negativos $\lambda_{1}, \ldots, \lambda_{t}$ e circuitos distintos $C_{1}, \ldots, C_{t}$ tais que $p(e)=\sum\left(\lambda_{i} \mid e \in C_{i}\right)$, para cada arco/aresta $e$ de $M$. Assim, o PCFC consiste em decidir se $(M, p)$ admite uma cobertura fracionária por circuitos.

Estamos agora em condições de começar o estudo do PCEC e do PCFC.

\subsection{PCEC/PCFC em grafos orientados}

Em um grafo orientado $M=D$, tanto o PCEC quanto o PCFC são relativamente fáceis de resolver: basicamente um par $(D, p)$ admite uma cobertura (fracionária) por circuitos se $p$ satisfaz a lei de Kirchhoff.

Proposição 2.3.1. Sejam $D$ um grafo orientado e $p: A(D) \mapsto \mathbb{Q}_{+}$. Então $(D, p)$ admite uma cobertura fracionária por circuitos se e somente se $p\left(\delta^{-}(v)\right)=p\left(\delta^{+}(v)\right)$ para todo $v \in V(D)$. Além disso, se $p$ for inteiro e valer a última condição então $(D, p)$ admite uma cobertura exata por circuitos. 
Baseado nestà simples caracterização, o PCEC e o PCFC podem ser resolvidos em tempo polinomial. Ainda, pode-se encontrar uma cobertura exata (fracionária) por circuitos de $(D, p)$, se ela existir, em tempo polinomial.

No contexto de fluxos em redes, um vetor $p$ satisfazendo a lei de Kirchhoff é chamado de circulação (em $D$ ). O seguinte resultado é um teorema bem conhecido demonstrado por Hoffman [42] envolvendo circulações.

Teorema 2.3.1 (Hoffman, 1960). Sejam D um grafo orientado e $l, u: A(D) \mapsto \mathbb{Q}_{+}$ capacidades tais que $l \leq u$. Então existe uma circulação $p$ em $D$ tal que $l \leq p \leq u$ se, $e$ somente se,

$$
l\left(\delta^{+}(X)\right) \leq u\left(\delta^{-}(X)\right) \text { para todo } X \subseteq V(D) .
$$

Se, além disso, $l$, u forem inteiros e valer a condição de corte então $p$ pode ser escolhido inteiro.

\subsection{PCEC/PCFC em grafos não-orientados}

Em grafos não-orientados o PCFC e o PCEC apresentam comportamentos diferenciados, ao contrário do que ocorre na versão orientada.

Comecemos nossa discussão com o PCFC. Sejam $G$ um grafo não-orientado e $p$ : $E(G) \mapsto \mathbb{Q}_{+}$. Uma condição necessária para que $(G, p)$ admita uma cobertura fracionária por circuitos é que

$$
p(\delta(X)-e) \geq p(e) \quad \text { para todo } X \subseteq V(G), e \in \delta(X) .
$$

Dizemos que um par $(G, p)$ que satisfaz a condição acima é balanceado.

Seymour [65] provou que a condição de $(G, p)$ ser balanceado é suficiente para a existência de uma cobertura fracionária por circuitos, obtendo assim uma boa caracterização para o PCFC.

Teorema 2.4.1 (Seymour, 1979). Sejam $G$ um grafo não-orientado e $p: E(G) \mapsto$ $\mathbb{Q}_{+}$. Então $(G, p)$ admite uma cobertura fracionária por circuitos se, e somente se, é balanceado.

Um algoritmo polinomial para a versão de busca do PCFC baseado na prova de Seymour foi desenvolvido por Arkin e Papadimitriou [10, 8].

Seymour também provou um resultado análogo ao Teorema de Hoffman para o caso não-orientado usando o Teorema 2.4.1.

Teorema 2.4.2. Sejam $G$ um grafo não-orientado e l, $u: E(G) \mapsto \mathbb{Q}_{+}$capacidades tais que $l \leq u$. Então existe $p: E(G) \mapsto \mathbb{Q}_{+}$tal que $l \leq p \leq u$ e $(G, p)$ admite uma cobertura fracionária por circuitos se, e somente se,

$$
u(\delta(X)-e) \geq l(e) \quad \text { para todo } X \subseteq V(G), e \in \delta(X) .
$$


Note que o Teorema 2.4.1 é um caso particular do resultado acima com $l=u=p$. $\mathrm{Na}$ verdade, pode-se mostrar que este último é uma conseqüência do primeiro [65].

Como já mencionamos, ao contrário do caso orientado, a versão fracionária é bem diferente da versão inteira. Vimos na Proposição 2.3.1 e no Teorema 2.3.1 que a condição adicional de $p$ ser inteiro é suficiente para a existência de uma cobertura exata por circuitos, porém, o mesmo não é verdade nos dois teoremas anteriores. Tome $G=K_{4}$ e $p \equiv 1$; o $\operatorname{par}(G, p)$ admite uma cobertura fracionária por circuitos, mas não uma cobertura exata por circuitos.

Claramente, é necessário que o par $(G, p)$ seja euleriano, isto é, que a condição

$$
p(\delta(X)) \text { é par para todo } X \subseteq V(G)
$$

esteja satisfeita para que $(G, p)$ admita uma cobertura exata por circuitos.

Vamos dizer que $(G, p)$ é admissível se for balanceado e euleriano. Assim, admissibilidade é uma condição necessária para a existência de uma cobertura exata por circuitos. Entretanto, isto não é sempre suficiente como mostra o seguinte contra-exemplo: seja $P_{10}$ o grafo de Petersen e tome $F$ como sendo um emparelhamento perfeito de $P_{10}$; defina $p_{10}(e)=2$ para $e \in F$, e $p_{10}(e)=1$, caso contrário (veja a Figura 2.1). Não é difícil ver

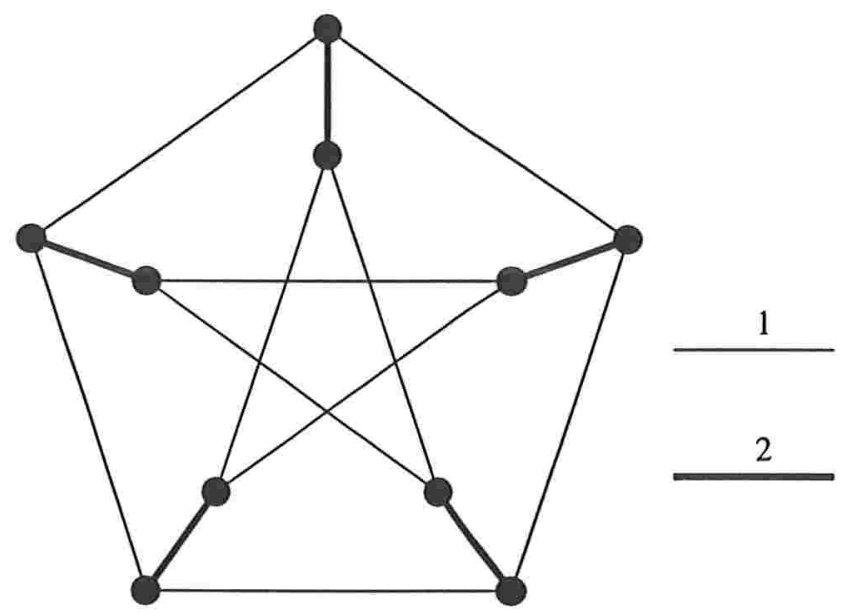

Figura 2.1: Grafo de Petersen.

que $\left(P_{10}, p_{10}\right)$ é admissível. De fato, suponha que $\left(P_{10}, p_{10}\right)$ admita uma cobertura exata por circuitos. Como em todo vértice incide exatamente uma aresta de $F$, cada circuito desta cobertura deve ser $F$-alternante e portanto de comprimento 8. Mas o peso total de $\left(P_{10}, p_{10}\right)$ é 20 , que não é um múltiplo de 8 . Portanto, $\left(P_{10}, p_{10}\right)$ não admite uma cobertura exata por circuitos.

Seymour [65] provou que se $G$ for planar a condição adicional acima é suficiente.

Teorema 2.4.3 (Seymour, 1979). Sejam $G$ um grafo planar e $p: E(G) \mapsto \mathbb{Z}_{+}$. Então $(G, p)$ admite uma cobertura exata por circuitos se, e somente se, é admissível. 
Alspach, Goddyn e Zhang [2] estenderam o resultado acima mostrando que $\left(P_{10}, p_{10}\right)$ é um contra-exemplo minimal. Denote por $\Re$ o conjunto de todos os pares admissíveis $(G, p)$ (a menos de isomorfismo). Defina a seguinte ordem parcial $\preceq$ em $\Re:\left(G_{1}, p_{1}\right) \preceq$ $\left(G_{2}, p_{2}\right)$ se $G_{1}$ é um menor de $G_{2}$ e $p_{1}(e) \leq p_{2}(e)$ para cada $e \in E\left(G_{1}\right) \subseteq E\left(G_{2}\right)$. Dizemos ainda que $\left(G_{1}, p_{1}\right)$ é menor que $\left(G_{2}, p_{2}\right)$ se $\left(G_{1}, p_{1}\right) \preceq\left(G_{2}, p_{2}\right)$ e $\left(G_{1}, p_{1}\right) \neq\left(G_{2}, p_{2}\right)$. Um par $(G, p) \in \Re$ é chamado de contra-par se não admite uma cobertura exata por circuitos. Um contra-par minimal é um contra-par que é minimal com relação à ordem parcial $\preceq$ definida acima.

Teorema 2.4.4 (Alspach, Goddyn e Zhang, 1994). $O$ par $\left(P_{10}, p_{10}\right)$ é o único contra-par minimal em $\Re$. Em particular, se $G$ é livre de Petersen então $(G, p)$ admite uma cobertura exata por circuitos se, e somente se, é admissivel.

Apresentamos aqui a demonstração de Alspach e Zhang [3] do teorema acima para o caso particular em que os grafos são cúbicos e $p$ assume apenas valores 1 e 2 . Este resultado precedeu o Teorema 2.4 .4 e serviu como ponto de partida para a demonstração deste.

Para apresentarmos essa prova precisamos antes introduzir algumas definições. Lembramos que um ciclo de um grafo $G$ é um subgrafo deste onde todos os vértices têm grau par. Definimos uma cobertura exata por ciclos de um par $(G, p)$ de modo análogo a uma cobertura exata por circuitos. É fácil ver que um par $(G, p)$ admite uma cobertura exata por circuitos se, e somente se, admite uma cobertura exata por ciclos.

Dizemos que um par admissível (euleriano) $(G, p)$ é $(1,2)$-admissível (-euleriano) se $p$ assume apenas valores 1 e 2 . Note que um par $(1,2)$-euleriano $(G, p)$ é admissível se, e somente se, $G$ não contém pontes. Dado um par $(G, p)$ fixo, denotamos por $E_{i}$ o conjunto das arestas com peso $i$, ou seja, $E_{i}=\{e \in E(G) \mid p(e)=i\}$, para $i=1,2$. Note que $E_{1}$ é um ciclo de $G$. Usamos o símbolo $\triangle$ para denotar a operação de diferença simétrica entre conjuntos.

Lema 2.4.1. Todo par $(1,2)$-admissível $(G, p)$ tal que $G$ é um grafo cúbico 3-arestacolorivel admite uma cobertura exata por circuitos.

Prova. Fixe uma 3-aresta-coloração própria de $G$ com cores 1,2 e 3 . Denote por $C_{i, j}$ o conjunto das arestas de cor $i$ ou $j$. Note que cada $C_{i, j}$ é um ciclo de $G$. Então não é difícil ver que

$$
\left\{C_{1,2} \triangle E_{1}, C_{1,3} \triangle E_{1}, C_{2,3} \triangle E_{1}\right\}
$$

é uma cobertura exata por ciclos de $(G, p)$.

Seja $(G, p)$ um par $(1,2)$-admissível e suponha que $y$ seja um vértice de grau 2 em $G$ e $x y, y z \in E(G)$. Note que $p(x y)=p(y z)$. Claramente, o par $(G / x y, p)$ é admissível, e ainda, $(G / x y, p)$ admite uma cobertura exata por circuitos se, e somente se, $(G, p)$ admite uma cobertura exata por circuitos. Lembramos que $\widetilde{G}$ denota o grafo obtido a partir de $G$ substituindo cada caminho maximal $P$ no qual todos seus vértices internos 
têm grau dois por uma aresta ligando os extremos de $P$. Assim, em vista da observação anterior, temos o seguinte resultado.

Proposição 2.4.1. Seja $(G, p)$ um par $(1,2)$-admissível. Então, $(\widetilde{G}, p)$ admite uma cobertura exata por circuitos se, e somente se, $(G, p)$ admite uma cobertura exata por circuitos.

Dizemos que $G$ é um grafo de permutação se este é cúbico e contém um 2-fator que consiste de dois circuitos ímpares $C_{1}, C_{2}$ sem cordas. Em particular, $E(G)-\left(C_{1} \cup C_{2}\right)$ é um emparelhamento perfeito de $G$. Um exemplo de grafo de permutação é o grafo de Petersen. O seguinte resultado foi demonstrado por Ellingham [28] e é necessário na demonstração do Teorema 2.4.6.

Teorema 2.4.5 (Ellingham, 1984). Seja $G$ um grafo de permutação com um 2-fator consistindo de dois circuitos ímpares sem cordas $C_{1}, C_{2}$ e seja $F:=E(G)-\left(C_{1} \cup C_{2}\right)$. Suponha que $G$ seja livre de Petersen. Então:

(a) G contém um quadrado que possui exatamente duas arestas de F;

(b) $G$ é hamiltoniano;

(c) Gé 3-aresta-colorível.

Além disso, se $G$ não é 3-aresta-colorivel então existe $F^{\prime} \subset F$ tal que $G-F^{\prime}$ é uma subdivisão do grafo de Petersen.

Note que no teorema acima temos $(\mathrm{a}) \Rightarrow(\mathrm{b}) \Rightarrow$ (c).

Estamos agora em condições de demonstrar o teorema de Alspach e Zhang.

Teorema 2.4.6 (Alspach e Zhang, 1993). Seja $\Re^{\prime}$ o conjunto de todos os pares $(1,2)$-admissiveis $(G, p)$ onde $G$ é cúbico. Então $\left(P_{10}, p_{10}\right)$ é o único contra-par minimal em $\Re^{\prime}$.

Prova. Seja $(G, p)$ um contra-par minimal em $\Re^{\prime}$. Então queremos mostrar que $(G, p)=\left(P_{10}, p_{10}\right)$. Note que, pela minimalidade de $(G, p)$, qualquer par de $\Re^{\prime}$ menor que $(G, p)$ admite uma cobertura exata por circuitos.

Como $(G, p)$ é admissível e minimal por hipótese, temos que $G$ é 2-aresta-conexo. Vamos mostrar que $G$ é 3-aresta-conexo. Para isto, suponha que $\left\{e, e^{\prime}\right\}$ seja um corte de arestas de $G$ tal que $G-\left\{e, e^{\prime}\right\}$ possui dois componentes $X$ e $Y$. Sejam $e=x y$ e $e^{\prime}=x^{\prime} y^{\prime}$ com $x, x^{\prime} \in V(X)$ e $y, y^{\prime} \in V(Y)$. Se $x=x^{\prime}$ então a outra aresta incidente a $x$ é uma ponte, o que contraria o fato de $G$ ser 2 -aresta-conexo. Portanto, $x \neq x^{\prime}$. Analogamente, temos $y \neq y^{\prime}$.

Como $X$ e $Y$ são conexos, temos que $G_{X}:=X+x x^{\prime}$ e $G_{Y}:=Y+y y^{\prime}$ são grafos cúbicos 2-aresta conexos. Atribua peso $p(e)\left(=p\left(e^{\prime}\right)\right)$ a $x x^{\prime}$ e $y y^{\prime}$ e considere os pares $\left(G_{X}, p\right)$ e $\left(G_{Y}, p\right)$. Ambos pertencem a $\Re$, isto é, são $(1,2)$-admissíveis, e são menores que $(G, p)$. Portanto, $\left(G_{X}, p\right)$ e $\left(G_{Y}, p\right)$ admitem coberturas exatas por circuitos $\mathcal{F}_{X}$ e 
$\mathcal{F}_{Y}$, respectivamente. Como as arestas $x x^{\prime}$ e $y y^{\prime}$ têm o mesmo peso, podemos removê-las dos circuitos de $\mathcal{F}_{X}$ e $\mathcal{F}_{Y}$ no qual elas aparecem e conectar, de modo apropriado, os $2 p(e)$ caminhos resultantes com as arestas $x y$ e $x^{\prime} y^{\prime}$ para formar $p(e)$ circuitos em $G$. Estes circuitos juntamente com os circuitos de $\mathcal{F}_{X} \cup \mathcal{F}_{Y}$ que não contêm $x x^{\prime}$ nem yy formam uma cobertura exata de $(G, p)$, contrariando o fato deste ser um contra-par. Logo, $G$ é 3 -aresta-conexo.

Como $G$ é cúbico, as arestas de $E_{1}$ formam circuitos disjuntos nos vértices e cada vértice é incidente a pelo menos uma aresta de peso 2 . Queremos mostrar que $E_{1}$ consiste de exatamente dois circuitos ímpares $C_{1}$ e $C_{2}$, e que toda aresta $x y \in E_{2}$ liga um vértice de $C_{1}$ a um vértice de $C_{2}$. Seja $e=x y$ uma aresta de peso 2 e considere $G_{e}:=G-e$. Como $G$ é 3-aresta-conexo, $G_{e}$ é 2 -aresta-conexo. Portanto, $\widetilde{G}_{e}$ é um grafo cúbico 2-aresta-conexo e $\left(\widetilde{G}_{e}, p\right)$ é um par $(1,2)$-admissível em $\Re$ menor que $(G, p)$. Pela minimalidade de $(G, p)$, segue que $\left(\widetilde{G_{e}}, p\right)$ admite uma cobertura exata por circuitos, e pela Proposição 2.4.1, $\left(G_{e}, p\right)$ também.

Seja $\mathcal{F}$ uma cobertura exata por circuitos de $\left(G_{e}, p\right)$. Tome um conjunto minimal $\mathcal{C}:=\left\{C_{0}, \ldots, C_{t}\right\}$ de circuitos de $\mathcal{F}$ tal que a união destes é conexa e contém $x$ e $y$ (possivelmente $\mathcal{C}$ pode conter um único circuito). Pela minimalidade de $\mathcal{C}$, é claro que podemos supor que $x \in V\left(C_{0}\right), y \in V\left(C_{t}\right)$ e $V\left(C_{i}\right) \cap V\left(C_{j}\right)=\emptyset$ se $i \neq j$ e $i \neq j \pm 1$.

Vamos definir um novo par $(1,2)$-admissível $\left(G^{\prime}, p^{\prime}\right)$ como segue. A união dos circuitos $C_{0}, \ldots, C_{t}$ junto com a aresta $e=x y$ forma o grafo $G^{\prime}$. Para $f \neq e$, definimos $p^{\prime}(f)$ como o número de circuitos de $\mathcal{F}$ que contêm $f$ e definimos $p^{\prime}(e):=2$. Claramente, $\left(\widetilde{G}^{\prime}, p^{\prime}\right)$ é $(1,2)$-admissível pois $G^{\prime}$ é 2-aresta-conexo, e $\left(\widetilde{G^{\prime}}, p^{\prime}\right) \preceq(G, p)$. Se $\mathcal{C} \neq \mathcal{F}$ então $\left(\widetilde{G^{\prime}}, p^{\prime}\right)$ é menor que $(G, p)$ e admite uma cobertura exata por circuitos, e pela Proposição 2.4.1, $\left(G^{\prime}, p^{\prime}\right)$ admite uma cobertura exata por circuitos, digamos $\mathcal{F}^{\prime}$. Então $\mathcal{F}^{\prime} \cup(\mathcal{F}-\mathcal{C})$ é uma cobertura exata por circuitos de $(G, p)$, o que contraria o fato deste ser um contra-par. Portanto, $\mathcal{F}=\mathcal{C}=\left\{C_{0}, \ldots, C_{t}\right\}$ e $\left(G^{\prime}, p^{\prime}\right)=(G, p)$.

Se $t=0$ então $G$ consiste de três arestas paralelas e $(G, p)$ não pode ser um contra-par. Logo, $t>0$. Sejam

$$
E^{0}:=\left\{f \in C_{i} \mid i \text { é par }\right\}
$$

e

$$
E^{1}:=\left\{f \in C_{i} \mid i \text { é ímpar }\right\} .
$$

Atribua cor amarela às arestas de $E^{0}-E^{1}$, cor azul às arestas de $E^{1}-E^{0}$ e cor verde às arestas de $E^{0} \cap E^{1}$. Isto define de modo natural uma 3-aresta-coloração própria de $\widetilde{G_{e}}$. Entretanto, ela não é uma 3-aresta-coloração própria de $G-e$ pois as arestas incidentes a $x$ têm mesma cor e as arestas incidentes a $y$ têm mesma cor. Note que o conjunto de todas as arestas coloridas em amarelo e azul é exatamente o conjunto das arestas de peso 1 e o conjunto das arestas coloridas em verde é exatamente o conjunto das arestas de peso 2 distintas de $e$.

Se $x$ e $y$ estão contidos em um mesmo componente do subgrafo induzido pelas arestas amarelas e azuis de $G-e$, então existe um caminho $P$ amarelo-azul alternante ligando os dois vértices. Permutando as cores neste caminho e atribuindo cor verde a $e$ obtemos 
uma 3-aresta-coloração própria de $G$. Pelo Lema 2.4.1, temos que $(G, p)$ admite uma cobertura exata por circuitos, o que contraria o fato deste ser um contra-par.

Portanto, $x$ e $y$ pertencem a componentes distintos do subgrafo induzidos pelas arestas amarelas e azuis de $G-e$. Esses dois componentes são circuitos ímpares $C_{1}$ e $C_{2}$ em $G$ e todos os outros componentes são circuitos pares. Por outro lado, as arestas de peso 1 são exatamente as arestas de $G-e$ coloridas em amarelo e azul, e $e$ é uma aresta de peso 2 de $G$ escolhida arbitrariamente. Logo, todas as arestas de peso 2 de $G$ ligam um vértice de $C_{1}$ a um vértice de $C_{2}$. Portanto, $G$ é um grafo de permutação.

Como $G$ não é 3-aresta-colorível por ser um contra-par, pelo Teorema 2.4 .5 existe $S \subset E_{2}$ tal que $G-S$ é uma subdivisão do grafo de Petersen. É fácil ver então que $\left(P_{10}, p_{10}\right) \preceq(G, p)$. Pela minimalidade de $(G, p)$, temos que $(G, p)=\left(P_{10}, p_{10}\right)$. Isto conclui a prova.

A idéia da demonstração do Teorema 2.4.4 (omitida aqui) para o caso mais geral é basicamente a seguinte. Inicialmente mostra-se que todo contra-par minimal $(G, p)$ em $\Re$ têm grau no máximo 4 e $p$ assume apenas valores 1 e 2 . A parte mais complicada consiste em mostrar que $G$ é cúbico, e então o resultado segue do Teorema 2.4.6.

A demonstração desses autores não fornece (ao menos, não de modo imediato) um algoritmo polinomial para encontrar uma cobertura exata por circuitos de um par admissível $(G, p)$, onde $G$ é livre de Petersen. Entretanto, eles mostraram que um algoritmo polinomial para este problema seguiria de um algoritmo polinomial para o seguinte caso especial.

\section{Problema Chave.}

Entrada: Um par $(1,2)$-admissível $(G, p)$ tal que $G$ é um grafo não-orientado livre de Petersen com grau máximo 4.

Saída: Uma cobertura exata por ciclos (ou circuitos) de $(G, p)$.

Não se conhece um algoritmo polinomial para o Problema Chave mesmo quando $G$ é cúbico. A demonstração do Teorema 2.4.6 vista acima usa um forte argumento de simetria e parece difícil transformá-la em um algoritmo polinomial.

Aqui cabe mencionar a relação do PCEC para grafos livres de Petersen com duas conjecturas famosas de Tutte sobre 4-fluxos. Um k-fluxo (não-nulo em toda parte) ${ }^{1}$ de um grafo $G$ é um par $(D, f)$ onde $D$ é uma orientação de $G$ e $f$ é uma circulação em $D$ que assume apenas valores em $\{ \pm 1, \pm 2, \ldots, \pm(k-1)\}$. É um fato bem conhecido que $G$ admite um 4-fluxo se, e somente se, existem ciclos $C_{1}, C_{2}$ de $G$ tal que $E(G)=C_{1} \cup C_{2}$. Além disso, se $G$ é cúbico então $G$ admite um 4 -fluxo se, e somente se, $G$ é 3 -arestacolorível.

\footnotetext{
${ }^{1}$ Nowhere-zero $k$-flow
} 
As duas conjecturas seguintes são problemas clássicos na teoria dos grafos.

Conjectura 2.4.1 (Tutte $[71], 1966)$. Todo grafo cúbico 2-aresta-conexo livre de Petersen admite um 4-fluxo. Ou, equivalentemente, todo grafo cúbico 2-aresta-conexo livre de Petersen é 3-aresta-colorível.

Conjectura 2.4.2 (Tutte [72], 1967). Todo grafo 2-aresta-conexo livre de Petersen admite um 4-fluxo.

O seguinte lema, basicamente uma generalização do Lema 2.4.1, mostra como os conceitos de 4 -fluxo e cobertura exata por circuitos estão relacionados.

Lema 2.4.2. Seja $(G, p)$ um par (1,2)-admissivel tal que $G$ admite um 4-fluxo $C_{1}, C_{2} e$ $E_{1}=\{e \in E(G) \mid p(e)=1\}$. Então

$$
\left\{C_{1} \triangle E_{1}, C_{2} \triangle E_{1},\left(C_{1} \triangle C_{2}\right) \triangle E_{1}\right\}
$$

é uma cobertura exata por ciclos de $(G, p)$.

Com base no Lema 2.4.2, se a Conjectura 2.4.2 for verdadeira e soubermos obter um 4-fluxo em tempo polinomial, então obteríamos um algoritmo polinomial para o Problema Chave. Note que para grafos arbitrários, o problema de decidir se um grafo cúbico é 3aresta-colorível (ou equivalentemente, se admite um 4-fluxo) é NP-completo [41].

Em 1979, Jaeger [45] provou o seguinte resultado.

Teorema 2.4.7 (Jaeger, 1979). Todo grafo 4-aresta-conexo admite um 4-fluxo.

Um algoritmo polinomial para encontrar um 4-fluxo em um grafo 4-aresta-conexo foi descrito por Jackson [44]. Em vista deste resultado, segue-se que todo par $(1,2)$ admissível $(G, p)$, onde $G$ é 4 -aresta-conexo, admite uma cobertura exata por circuitos. Isto implica que o PCEC para grafos 4-aresta-conexos com pesos 1 e 2 pode ser resolvido em tempo polinomial.

Para grafos planares sabe-se que as Conjecturas 2.4 .1 e 2.4.2 são verdadeiras (e equivalentes). Elas correspondem ao famoso Teorema das Quatro Cores, demonstrado por Appel e Haken $[4,5,6]$ usando um computador para efetuar vários cálculos.

Teorema 2.4.8 (Appel e Haken, 1977). Todo grafo planar é 4-colorível. Ou equivalentemente, todo grafo cúbico planar 2-aresta-conexo é 3-aresta-colorível.

O leitor interessado pode consultar [47] para obter uma explicação mais detalhada dessa famosa demonstração. Ressaltamos que, recentemente, Robertson, Sanders, Seymour e Thomas [59] simplificaram a demonstração de Appel e Haken, mas ainda tiveram de utilizar computadores. No entanto, sua demonstração pode ser vista como um algoritmo polinomial para 4-colorir os vértices de um grafo planar (isso é equivalente a encontrar um 4-fluxo). Logo, o Problema Chave restrito a grafos planares pode ser resolvido em tempo 
polinomial. Usando este fato, pode-se mostrar que o problema de encontrar coberturas exatas por circuitos em grafos planares pode ser resolvido em tempo polinomial.

Mais recentemente, ficamos sabendo que Robertson, Sanders, Seymour e Thomas anunciaram uma demonstração da Conjectura 2.4.1. A demonstração consiste de duas partes:

1. Mostrar que é suficiente provar a conjectura para dois tipos de grafos cúbicos quase planares [60]: apex graphs (grafos que possuem um vértice cuja remoção torna o grafo resultante planar) e doublecross graphs (grafos que podem ser desenhados no plano com apenas dois cruzamentos na face externa);

2. Adaptar a (nova) prova do Teorema das Quatro Cores [59] para mostrar que grafos cúbicos com as propriedades acima são 3 -aresta-coloríveis.

A demonstração fornece um algoritmo polinomial para 3-colorir as arestas de um grafo cúbico livre de Petersen. Uma conseqüência imediata disso é a existência de um algoritmo polinomial para o PCEC em grafos cúbicos livre de Petersen. Note que isso não resolve o Problema Chave a não ser que saibamos reduzi-lo ao caso cúbico.

Uma questão pertinente é quanto a existência de algoritmos polinomiais para o PCEC em grafos planares (livres de Petersen) que não dependam de algoritmos polinomiais para encontrar 4-fluxos, uma vez que é possível demonstrar os Teoremas 2.4 .3 e 2.4.4 independentemente do Teorema das Quatro Cores e da Conjectura 2.4.1 de Tutte.

Para grafos não-orientados arbitrários, não se conhece a complexidade computacional do PCEC. Além disso, para pesos potencialmente grandes não é claro que se um par $(G, p)$ admite uma cobertura exata por circuitos, então este admite uma cobertura exata com um número polinomial (no tamanho de $(G, p)$ ) de circuitos, mesmo usando a representação mais compacta mencionada na Seção 2.2. Veremos na Seção 2.8 que para grafos livres de Petersen, um par admissível $(G, p)$ admite uma cobertura exata por circuitos com um número polinomial de circuitos.

\subsection{PCEC/PCFC em grafos mistos}

Agora começamos o estudo do problema da cobertura (fracionária) por circuitos em grafos mistos. Em 1986 Arkin e Papadimitriou [10, 8] demonstraram o seguinte.

Teorema 2.5.1 (Arkin e Papadimitriou, 1986). O PCEC e o PCFC são problemas NP-completos para grafos mistos.

Em vista deste resultado, é improvável que exista uma boa caracterização para a existência de uma cobertura exata por circuitos em um grafo misto. Nesta seção mostramos que para grafos mistos série-paralelos tal caracterização existe, e na próxima descrevemos um algoritmo polinomial (baseado no método dos elipsóides) para encontrar coberturas exatas/fracionárias por circuitos, se estas existirem. 
As seguintes condições são claramente necessárias para a existência de uma cobertura fracionária por circuitos de $(M, p)$ :

(a) Para todo $X \subseteq V, f_{p}(X):=p(\delta(X))-\left|p\left(\delta^{-}(X)\right)-p\left(\delta^{+}(X)\right)\right| \geq 0$;

(b) Para todo corte $\nabla(X)$ e $e \in \delta(X), p(\nabla(X)-e) \geq p(e)$.

Ainda, se $(M, p)$ admite uma cobertura exata por circuitos então é necessário que $p$ seja inteiro não-negativo e a seguinte condição seja satisfeita:

(c) Para todo corte $\nabla(X), p(\nabla(X))$ é par.

Dizemos que $(M, p)$ é balanceado se satisfaz (b); euleriano ${ }^{2}$ se satisfaz (a) e (c); e fracionariamente admissível se satisfaz (a) e (b). Se $p$ for inteiro, dizemos que $(M, p)$ é admissível se for euleriano e balanceado (ou, se for fracionariamente admissível e (c) for satisfeita).

O seguinte exemplo mostra que admissibilidade (fracionária) não é sempre suficiente para garantir a existência de uma cobertura exata (fracionária) por circuitos. Tome o grafo misto $M K_{4}$ cujo grafo subjacente é isomorfo a $K_{4}$, onde a parte orientada consiste de um circuito orientado de comprimento 4 e a parte não-orientada consiste de um 1fator. Atribua peso 1 aos arcos e peso 2 às arestas de $M K_{4}$ (veja a Figura 2.2).



Figura 2.2: Grafo $M K_{4}$.

É fácil ver que $M K_{4}$ com esses pesos é (fracionariamente) admissível mas não admite uma cobertura exata (fracionária) por circuitos.

O principal resultado desta seção é a demonstração de que admissibilidade é uma condição suficiente para a existência de uma cobertura exata por circuitos quando $M$ é série-paralelo.

Descrevemos a seguir dois lemas que serão necessários para demonstrar o resultado principal. A prova do primeiro é simples e fica a cargo do leitor.

\footnotetext{
${ }^{2}$ Esta nomenclatura vem por analogia com a nossa definição de grafo misto euleriano - veja o Capítulo 1.
} 
Lema 2.5.1. Seja $G$ um grafo não-orientado série-paralelo, $B$ um corte minimal de $G$ e $C$ um circuito de $G$. Então $|B \cap C| \leq 2$.

Lema 2.5.2. Seja $(M, p)$ um par admissivel. Considere um subconjunto $X \subseteq V(M) e$ uma aresta $e=u v \in \delta(X)$, com $u \in X$, tais que $p(e)>0, f_{p}(X)=0$ e $p\left(\delta^{-}(X)\right)-$ $p\left(\delta^{+}(X)\right)>0$. Seja $M^{\prime}$ o grafo obtido a partir de $M$ substituindo e pelo arco $a=(u, v) ; e$ seja $p^{\prime}$ a função peso em $M^{\prime}$, obtida de $p$ definindo $p^{\prime}(a):=p(e)$. Então o par resultante $\left(M^{\prime}, p^{\prime}\right)$ é admissivel.

Prova. Inicialmente provaremos o lema para o caso $p(e)=1$. Suponha por contradição que $\left(M^{\prime}, p^{\prime}\right)$ não seja admissível. Claramente, as condições (b) e (c) são satisfeitas por $\left(M^{\prime}, p^{\prime}\right)$; portanto, existe algum subconjunto $Y \subseteq V(M)$ tal que $e \in \delta(Y)$ e

$$
f_{p^{\prime}}(Y):=p\left(\delta_{M^{\prime}}(Y)\right)-\left|p\left(\delta_{M^{\prime}}^{-}(Y)\right)-p\left(\delta_{M^{\prime}}^{+}(Y)\right)\right|<0 .
$$

Como $f_{p^{\prime}}(Y)=f_{p^{\prime}}(V(M)-Y)$, podemos supor sem perda de generalidade que $v \in Y$. Ou seja, o arco $a=(u, v)$ de $M^{\prime}$ entra em $Y$.

Para simplificar nossa apresentação, escrevemos $h(S):=p\left(\delta^{-}(S)\right)-p\left(\delta^{+}(S)\right)$ para todo subconjunto $S$ de $V(M)$. No que segue $h$ refere-se somente ao par $(M, p)$. Como $f_{p^{\prime}}(Y)=p(\delta(Y)-e)-|h(Y)+1|$, e $f_{p^{\prime}}(Y)<0$, temos

$$
p(\delta(Y)-e)<|h(Y)+1| \text {. }
$$

Primeiro suponha que $h(Y)<0$. Neste caso, $|h(Y)+1|=|h(Y)|-1$. Logo, usando a desigualdade (2.1) concluímos que $p(\delta(Y))-1<|h(Y)|-1$, e portanto $f_{p}(Y)=$ $p(\delta(Y))-|h(Y)|<0$, o que contraria a admissibilidade de $(M, p)$.

Assim, $h(Y) \geq 0$. Neste caso, como $p(e)=1$, a desigualdade (2.1) equivale a $p(\delta(Y))<h(Y)+2$.

Por hipótese, $f_{p}(X)=0$ e $h(X)>0$. Logo, $p(\delta(X))=h(X)$. Combinando esta igualdade com a desigualdade anterior para $p(\delta(Y))$ obtemos

$$
p(\delta(X))+p(\delta(Y))<h(X)+h(Y)+2 .
$$

Como $h$ é uma função modular, isto é, satisfaz $h(X)+h(Y)=h(X \cap Y)+h(X \cup Y)$, temos

$$
p(\delta(X))+p(\delta(Y))<h(X \cap Y)+h(X \cup Y)+2 .
$$

Agora, como

$$
\begin{aligned}
p(\delta(X))+p(\delta(Y)) & =p(\delta(X \cap Y))+p(\delta(X \cup Y))+2 p(\delta(X-Y, Y-X)), \\
h(X \cap Y) & \leq|h(X \cap Y)|=p(\delta(X \cap Y))-f_{p}(X \cap Y), \\
h(X \cup Y) & \leq|h(X \cup Y)|=p(\delta(X \cup Y))-f_{p}(X \cup Y),
\end{aligned}
$$

substituindo-os na desigualdade acima, derivamos a seguinte contradição:

$$
2=2 p(e) \leq 2 p(\delta(X-Y, Y-X))<-f_{p}(X \cap Y)-f_{p}(X \cup Y)+2 \leq 2 .
$$


Isto mostra que $\left(M^{\prime}, p^{\prime}\right)$ é admissível se $p(e)=1$.

Se $p(e) \geq 2$ substituímos a aresta $e$ por $p(e)$ arestas paralelas com extremos $u, v$, e atribuímos peso 1 a cada uma delas. Claramente, o par resultante $\left(M^{\prime \prime}, p^{\prime \prime}\right)$ é admissível. Usando o lema no caso de peso unitário (que acabamos de demonstrar) para $\left(M^{\prime \prime}, p^{\prime \prime}\right)$, concluímos que podemos substituir cada aresta paralela $u v$ por um arco $(u, v)$ e o novo par $\left(M^{\prime}, p^{\prime}\right)$ ainda é admissível. Finalmente, substituindo os $p(e)$ arcos paralelos $(u, v)$ por um arco $(u, v)$ com peso $p(e)$ ainda conservamos admissibilidade. Isto conclui a demonstração.

A demonstração do teorema principal é inspirada na demonstração de Seymour [65] para grafos não-orientados planares, e na demonstração do Teorema 2.4.4 de Alspach et al [2].

Teorema 2.5.2. Se $M=(V, E, A)$ é série-paralelo então $(M, p)$ admite uma cobertura exata por circuitos se, e somente se, é admissivel.

Prova. É imediato que se $(M, p)$ admite uma cobertura exata por circuitos então $p$ é admissível. Para provar o contrário, suponha que $(M, p)$ seja um par admissível e que $p(e)>0$ para todo $e \in E \cup A$ (senão podemos remover $e$ de $M$ ). Aplicando o Lema 2.5.2 podemos também supor que se $f_{p}(X)=0$ então não existe nenhuma aresta no corte $\nabla(X)$.

Usaremos indução em $p(E)$. Se $p(E)=0$ então $M$ é um grafo orientado e $p\left(\delta^{-}(v)\right)=$ $p\left(\delta^{+}(v)\right)$ para todo $v \in V$. O resultado é imediato. Suponha então que $p(E)>0$.

Suponha que exista um corte $\nabla(X)$ tal que $|\nabla(X)|=|\delta(X)|=2$, isto é o corte $\nabla(X)$ consiste de exatamente duas arestas, digamos, e e $f$. Sejam $M^{\prime}:=M / e$ e $p^{\prime}$ a restrição de $p$ a $M^{\prime}$. Claramente $\left(M^{\prime}, p^{\prime}\right)$ é admissível, e pela hipótese de indução, admite uma cobertura exata por circuitos, digamos, $\mathcal{F}$. Note que os $p(f)=p(e)$ circuitos de $\mathcal{F}$ que contêm $f$ também contêm o vértice $v_{e}$ correspondente à identificação dos extremos de $e$. Cada um desses circuitos corresponde a um circuito de $M$ contendo $e$ e $f$. Além disso, os demais circuitos de $F$ que contêm $v_{e}$ correspondem a circuitos de $M$ que contêm apenas um dos extremos de $e$. É fácil ver agora que $(M, p)$ admite uma cobertura exata por circuitos. Portanto, podemos supor que $M$ não contém nenhum corte formado por duas arestas.

Se existe uma aresta $e_{0}$ com $p\left(e_{0}\right)=1$ então podemos atribuir uma orientação arbitrária a $e_{0}$. Como $f_{p}(X) \geq 2$ para todo $\emptyset \neq X \subset V$ tal que $e_{0} \in \delta(X)$, segue que o par resultante $\left(M^{\prime}, p^{\prime}\right)$ é admissível. Usando a hipótese de indução o resultado segue. Assim, podemos supor que não existem arestas com peso 1 .

Seja $e_{0}$ uma aresta de peso máximo. Então $p\left(e_{0}\right) \geq 2$. Seja $p^{\prime}=p-2 \chi^{e_{0}}$. Afirmamos que $\left(M, p^{\prime}\right)$ é admissível. Claramente, $\left(M, p^{\prime}\right)$ é euleriano. Resta mostrar que $\left(M, p^{\prime}\right)$ é balanceado. Suponha por contradição que exista um corte $\nabla(X)$ tal que $p^{\prime}(\nabla(X)-e)<$ $p^{\prime}(e)$ para alguma aresta $e \in \delta(X)$. Claramente, $e_{0} \in \delta(X)$. Se $e=e_{0}$ então

$$
p\left(\nabla(X)-e_{0}\right)=p^{\prime}\left(\nabla(X)-e_{0}\right)<p^{\prime}\left(e_{0}\right)<p\left(e_{0}\right),
$$


o que contraria a admissibilidade de $(M, p)$. Portanto, $e \neq e_{0}$. Como $p^{\prime}(\nabla(X))$ é par, temos $p^{\prime}(\nabla(X)-e) \leq p^{\prime}(e)-2$. Por outro lado,

$$
p\left(e_{0}\right) \leq p(\nabla(X)-e)=p^{\prime}(\nabla(X)-e)+2 \leq p^{\prime}(e)=p(e) .
$$

Mas então $p\left(e_{0}\right)=p(e)$ e $|\nabla(X)|=|\delta(X)|=2$, contrariando a inexistência de tais cortes.

Logo, $\left(M, p^{\prime}\right)$ é admissível e $p^{\prime}(E)=p(E)-2$. Pela hipótese de indução, $\left(M, p^{\prime}\right)$ admite uma cobertura exata por circuitos, digamos, $\mathcal{F}$. Seja $\mathcal{F}=\mathcal{F}_{0} \cup \mathcal{F}_{1}$, onde os circuitos em $\mathcal{F}_{0}$ contêm $e_{0}=x y$ e os circuitos em $\mathcal{F}_{1}$ não contêm $e_{0}$.

Defina um grafo não-orientado auxiliar $H$ como segue. Tome $V(H):=V$ e para cada $C \in \mathcal{F}_{1}$ construa em $H$ um circuito $C$ que corresponde ao circuito subjacente de $C$. Rotule as arestas deste circuito com " $C$ ". Afirmamos que existe um caminho de $x$ a $y$ em $H$.

De fato, seja $X$ o conjunto dos vértices que são atingíveis por $x$ em $H$, e suponha que $y \notin X$. Seja $B$ um corte minimal de $M$ contido em $\nabla(X)$ tal que $e_{0} \in B$. Então nenhum circuito de $\mathcal{F}_{1}$ usa um arco ou uma aresta de $B$, e pelo Lema 2.5.1, todo circuito de $\mathcal{F}_{0}$ usa $e_{0}$ e exatamente um elemento de $B$ distinto de $e_{0}$. Mas então temos que $p\left(B-e_{0}\right)=p^{\prime}\left(B-e_{0}\right)=p^{\prime}\left(e_{0}\right)=p\left(e_{0}\right)-2$, o que contraria a admissibilidade de $(M, p)$. Logo, existe um caminho de $x$ a $y$ em $H$, e portanto, existe uma família $\left\{C_{1}, \ldots, C_{k}\right\}$ de circuitos de $M$ tal que $x \in V\left(C_{1}\right), y \in V\left(C_{k}\right)$ e $V\left(C_{i}\right) \cap V\left(C_{j}\right) \neq \emptyset$ se, e somente se, $|i-j|=1$, para $1 \leq i<j \leq k$.

Considere o subgrafo de $M$ formado pela aresta $e_{0}$ e pela união dos circuitos $C_{1}, \ldots, C_{k}$. Afirmamos que $\left|V\left(C_{i}\right) \cap V\left(C_{i+1}\right)\right|=1$ para $i=1, \ldots, k-1$. Suponha por contradição que existam circuitos $C=C_{i}$ e $C^{\prime}=C_{i+1}$ tais que $\left|V(C) \cap V\left(C^{\prime}\right)\right| \geq 2$. Neste caso, não é difícil mostrar que existem vértices distintos $a, b \in V(C) \cap V\left(C^{\prime}\right)$ e outros vértices distintos $u \in V(C)-V\left(C^{\prime}\right)$ e $v \in V\left(C^{\prime}\right)-V(C)$; e também um caminho $P_{x u}$ de $x$ a $u$, e um caminho $P_{y v}$ de $y$ a $v$, tais que $P_{x u} \cup C \cup C^{\prime} \cup P_{y v} \cup\left\{e_{0}\right\}$ contém um subgrafo homeomorfo a $K_{4}$. Note o papel dos vértices $a, b, u, v$ neste homeomorfismo. Isto é uma contradição já que $M$ é série-paralelo.

Logo, $\left|V\left(C_{i}\right) \cap V\left(C_{i+1}\right)\right|=1$, para $i=1, \ldots, k-1$. É fácil ver que $\bigcup C_{i}$ pode ser particionado em um caminho de $x$ a $y$ e um caminho de $y$ a $x$, digamos, $P^{\prime}$ e $Q^{\prime}$. Cada um destes caminhos juntamente com $e_{0}$ forma um circuito em $M$. Sejam $P$ e $Q$ esses circuitos. Então a coleção de circuitos $\mathcal{F}-\left\{C_{1}, \ldots, C_{k}\right\} \cup\{P, Q\}$ é uma cobertura exata por circuitos de $(M, p)$. Isto conclui a prova.

O teorema acima fornece imediatamente um resultado para o problema da cobertura fracionária por circuitos.

Corolário 2.5.1. Se $M$ é série-paralelo então $(M, p)$ admite uma cobertura fracionária por circuitos se, e somente se, é fracionariamente admissivel. 
Para concluir esta seção, note que a propriedade de "ser admissível" pode ser verificada em tempo polinomial. Para verificar a condição (c) precisamos apenas testar se $p(\nabla(v))$ é para todo $v \in V$. A condição (b) pode verificada da seguinte forma. Seja $H$ o grafo subjacente de $M$. Para cada aresta $e=u v$ em $M$ calcule o corte de peso mínimo $B_{e}$, que separa $u$ de $v$, com relação a $p$ em $H-e$. Se para algum par $\left(e, B_{e}\right)$ tivermos $p\left(B_{e}\right)<p(e)$, então (b) não é satisfeita, caso contrário ela é válida. Finalmente, podemos testar a condição (a) do seguinte modo. Primeiramente note que (a) é satisfeita se e somente se $q(X):=p(\delta(X))-p\left(\delta^{-}(X)\right)+p\left(\delta^{+}(X)\right) \geq 0$ para todo $X \subseteq V$. Seja $D=\left(V, A \cup A^{\prime}\right)$ o grafo orientado obtido a partir de $M$ substituindo cada aresta por dois arcos (de mesmos extremos) formando um circuito. Defina capacidades $l(a)=u(a)=p(a)$ para $a \in A$ e $l\left(a^{\prime}\right)=0, u\left(a^{\prime}\right)=p(e)$ para cada $a^{\prime} \in A^{\prime}$ pertencendo ao circuito que substituiu $e$. É claro que a condição (a) é satisfeita se e somente se $D, l, u$ satisfazem a condição de Hoffman (Teorema 2.3.1). Isto pode ser verificado usando uma única computação de fluxo máximo ([1], Sec. 6.7).

\subsection{Um algoritmo polinomial para o PCEC em um grafo misto série-paralelo}

Nesta seção descrevemos um algoritmo polinomial (baseado no método dos elipsóides) para encontrar uma cobertura exata por circuitos de um par admissível $(M, p)$, onde $M$ é série-paralelo.

Seja então $(M, p)$ um par admissível, onde $M=(V, E, A)$ é série-paralelo. Como vimos anteriormente, uma cobertura exata por circuitos de $(M, p)$ pode ser representada por um par $(L, \mu)$, onde $L$ é uma lista (sem repetições) de circuitos de $M$ e $\mu$ é um vetor indicando a multiplicidade de cada circuito.

Não é imediato qu todo par admissível $(M, p)$, onde $M$ é série-paralelo, admite uma lista $L$ com un número polinomial de circuitos. Entretanto, na Seção 2.8 provamos que existe uma tal lista $L$ com no máximo $m:=|E \cup A|$ circuitos. Infelizmente, nossa demonstração desse último resultado não fornece diretamente um algoritmo polinomial para encontrar uma tal cobertura exata por circuitos. De fato, ela depende do Teorema 2.5.2.

Porém, se não exigirmos que a cobertura use no máximo $m$ circuitos então podemos obter um algoritmo polinomial baseado no método dos elipsóides. Para isso, necessitamos de um algoritmo de separação polinomial que é interessante por si só: encontrar um circuito mínimo em um grafo misto série-paralelo com pesos arbitrários.

Descrevemos dois algoritmos para encontrar uma cobertura exata por circuitos. $\mathrm{O}$ primeiro é baseado na demonstração do Teorema 2.5.2 mas é pseudo-polinomial. O segundo é um elegante procedimento polinomial que usa o primeiro e baseia-se no algoritmo descrito em [2]. 


\section{Algoritmo CirCov1}

Entrada: Um par admissível $(M, p)$, onde $M=(V, E, A)$ é série-paralelo.

Saída: Uma cobertura exata por circuitos $\mathcal{F}$ de $(M, p)$.

1. Remova arcos e arestas de peso 0 . Contraia qualquer aresta que esteja em um corte $\nabla(X)$ tal que $|\nabla(X)|=|\delta(X)|=2$. Para toda aresta $e$ em um corte $\nabla(X)$ tal que $f_{p}(X)=0$, atribua uma orientação à aresta $e$ de acordo com o Lema 2.5.2.

2. Se $p(E)=0$, então devolva uma cobertura exata por circuitos $\mathcal{F}$ (para grafos orientados isto é fácil) e pare.

3. Se existir alguma aresta com peso 1, então atribua uma orientação arbitrária a ela. Chame CirCov1 recursivamente para encontrar uma cobertura exata por circuitos $\mathcal{F}$ do novo grafo. Devolva $\mathcal{F}$ e pare.

4. Seja $e_{0}=x y$ uma aresta com peso máximo. Chame CirCov1 recursivamente para encontrar uma cobertura exata por circuitos $\mathcal{F}$ de $\left(M, p-2 \chi^{e_{0}}\right)$.

5. Como na demonstração do Teorema 2.5.2, encontre uma coleção $\left\{C_{1}, \ldots, C_{k}\right\}$ de circuitos que não contêm $e_{0}$ tal que $x \in V\left(C_{1}\right), y \in V\left(C_{k}\right)$ e $\left|V\left(C_{i}\right) \cap V\left(C_{j}\right)\right|$ é igual a 1 , se $|i-j|=1$, e 0 caso contrário. Decomponha $\bigcup C_{i}$ em um $(x, y)$-caminho $P^{\prime}$ e um $(y, x)$-caminho $Q^{\prime}$. Sejam $P:=P^{\prime} \cup\left\{e_{0}\right\}$ e $Q:=Q^{\prime} \cup\left\{e_{0}\right\}$. Devolva a cobertura exata por circuitos $\mathcal{F}-\left\{C_{1}, \ldots, C_{k}\right\} \cup\{P, Q\}$ e pare.

O passo 1 requer $O(|E|)$ computações de fluxo máximo (corte mínimo). O passo 2 pode ser executado em tempo $O(|A| \cdot|V|)$. O número total de chamadas a CirCov1 é limitado por $p(E) / 2$ pois o peso total das arestas em cada par sucessivo $(M, p)$ é reduzido de 2. Então CirCov1 é um algoritmo pseudo-polinomial.

Mostramos agora como obter um algoritmo polinomial usando CirCov1. A idéia é formular o problema da cobertura exata por circuitos para $(M, p)$ como um problema de programação linear inteiro e resolver sua relaxação (ou seja, resolvemos a versão de busca do PCFC). Usamos então a parte fracionária da solução resultante para definir um novo peso $p^{\prime}$ (com entradas relativamente pequenas), e chamamos CirCov1 para resolver o problema da cobertura exata por circuitos para $\left(M, p^{\prime}\right)$. Uma cobertura exata por circuitos de $(M, p)$ é obtida juntando-se a cobertura parcial por circuitos correspondente à parte inteira da solução do programa linear e a cobertura exata por circuitos devolvida por CirCov1.

No que segue, $N$ denota a matriz de incidência dos circuitos de $M$ e 1 denota o vetor de $|\mathcal{C}|$ uns. 


\section{Algoritmo CirCov2}

Entrada: Um par admissível $(M, p)$, onde $M=(V, E, A)$ é série-paralelo.

Saida: Uma cobertura exata por circuitos $(L, \mu)$ de $(M, p)$, onde $L$ é uma lista contendo no máximo $2|E \cup A|-1$ circuitos e $\mu$ é um vetor de multiplicidade cujas entradas são limitadas por $r:=\max \{p(e) \mid e \in E \cup A\}$.

1. Encontre uma solução básica viável $\lambda=\left(\lambda_{C}\right)_{C \in \mathcal{C}}$ do seguinte problema de programação linear:

$$
\max \{\lambda \mathbf{1} \mid N \lambda=p, \lambda \geq 0\} .
$$

2. Sejam $\{\lambda\rfloor:=\left(\left\lfloor\lambda_{C}\right\rfloor\right)_{C \in \mathcal{C}}$ e $\{\lambda\}:=\lambda-\lfloor\lambda\rfloor$ as partes inteira e fracionária de $\lambda$, e seja $p^{\prime}:=N\{\lambda\}=p-N[\lambda]$. (Note que, como $p^{\prime}$ é uma combinação linear não-negativa de circuitos, $\left(M, p^{\prime}\right)$ é fracionariamente admissível. Como $p$ e $N\lfloor\lambda\rfloor$ são eulerianos, então $p^{\prime}$ também é euleriano. Logo, $\left(M, p^{\prime}\right)$ é admissível)

3. Chame CirCov1 com entrada $\left(M, p^{\prime}\right)$ para obter uma cobertura exata por circuitos $L^{\prime}$ de $\left(M, p^{\prime}\right)$.

4. Junte $L^{\prime}$ à cobertura exata por circuitos $(S,\lfloor\lambda\rfloor)$, onde $S:=\left\{C \in \mathcal{C} \mid\left\lfloor\lambda_{C}\right\rfloor>0\right\}$, e devolva a cobertura exata por circuitos resultante $(L, \mu)$. Pare.

Vamos mostrar que $|L|$ é polinomialmente limitado. Como $\lambda$ é uma solução básica, temos $|S| \leq|E \cup A|$. Além disso, $\left|L^{\prime}\right|+\lfloor\lambda\rfloor 1 \leq \lambda 1=[\lambda\rfloor 1+\{\lambda\} 1$, e logo, $\left|L^{\prime}\right| \leq\{\lambda\} 1$. Como cada entrada não nula em $\{\lambda\}$ é menor que 1 temos $\{\lambda\} 1<|E \cup A|$ e portanto $\left|L^{\prime}\right| \leq|E \cup A|-1$. Então $|L| \leq|S|+\left|L^{\prime}\right| \leq 2|E \cup A|-1$. Como $r=\max \left\{p^{\prime}(e) \mid e \in\right.$ $E \cup A\} \leq\left|L^{\prime}\right|<|E \cup A|$, concluímos que o passo 3 pode ser executado em tempo polinomial.

Resta mostrar como resolver o passo 1 em tempo polinomial em $|E \cup A| \log (r)$, apesar do número exponencial de variáveis $\lambda_{C}$. Para isto, considere o programa linear dual de (2.2): $\min \left\{p x \mid N^{T} x \geq 1\right\}$. O problema da separação para este PL é o seguinte: Dado um vetor racional $x$, ou certifique que $x$ satisfaz $N^{T} x \geq 1$, ou encontre uma desigualdade violada (um circuito em $(M, x)$ tendo peso menor que 1.)

Pelo Teorema 1.2.1 (do Capítulo 1), uma solução básica ótima de (2.2) pode ser encontrada pelo método dos elipsóides em tempo polinomialmente limitado por $|E \cup A| \mathrm{e}$ pelo tamanho de $p$, desde que saibamos resolver o problema da separação acima em tempo polinomialmente limitado por $|E \cup A|$ e pelo tamanho de $x$. Para isto, precisamos de um algoritmo polinomial que encontra um circuito mínimo no grafo misto série-paralelo $(M, x)$. Descrevemos um tal algoritmo na próxima subseção.

\subsubsection{Circuitos mínimos em grafos mistos série-paralelos}

Considere um grafo misto série-paralelo $(M, w)$, onde $w$ é uma função peso arbitrária. Como estamos interessados em encontrar circuitos mínimos, podemos supor que $M$ é 
fortemente conexo, possui ao menos um circuito e não possui vértices de corte. Observe que como $w$ pode ter entradas negativas, devemos lidar com circuitos negativos. É um fato conhecido que para grafos mistos arbitrários o problema de detectar circuitos negativos é NP-completo $[9,8]$.

Claramente, podemos computar em tempo polinomial um circuito mínimo de comprimento $2 \mathrm{em} M$. Então é suficiente descrever um algoritmo polinomial para encontrar um circuito mínimo de comprimento ao menos $3 \mathrm{em} M$. Para resolver o último problema podemos nos restringir a grafos orientados: substituímos cada aresta $e$ de $M$ por dois arcos reversos, cada um com peso $w(e)$. Então, resta descrever um algoritmo polinomial para o seguinte problema:

$\operatorname{MC3P}(D, w)$ : Dado um grafo orientado série-paralelo $(D, w)$, encontre, se existir, um circuito mínimo $C$ de comprimento ao menos 3 .

$\mathrm{O}$ algoritmo é baseado na existência de um vértice com exatamente 2 vizinhos. Chamamos tal vértice de especial. Um resultado (fácil de se provar) que usamos é o seguinte.

Lema 2.6.1. Se $G$ é um grafo série-paralelo 2-conexo com $|V(G)| \geq 3$ então $G$ possui um vértice com exatamente dois vizinhos.

Na descrição do algoritmo usamos as duas operações seguintes sobre um grafo orientado $(D, w)$. No que segue, $\left.w\right|_{D^{\prime}}$ denota a restrição de $w$ a $D^{\prime}$.

Remoção de Arco Paralelo - $\operatorname{RAP}(D, w)$

Considere dois arcos paralelos $a$ e $b$.

Defina $D^{\prime}:=D-b$ e $w(a):=\min \{w(a), w(b)\}$.

Devolva $\left(D^{\prime},\left.w\right|_{D^{\prime}}\right)$.

Eliminação de Vértice Especial $-\operatorname{EVE}(D, w ; v)$

( $D$ não possui arcos paralelos e $v$ é especial)

Sejam $x$ e $y$ os vizinhos do vértice especial $v$.

1. Se $(x, v),(v, x),(y, v),(v, y) \in A(D)$ então

defina $D^{\prime}:=D /\{(y, v),(v, y)\}$

$w(x, v):=w(x, v)+w(v, y) ; w(v, x):=w(v, x)+w(y, v) ;$

2. Se $(x, v),(v, x),(v, y) \in A(D)$ e $(y, v) \notin A(D)$ então

defina $D^{\prime}:=(D-(v, x)) /(v, y) ; w(x, v):=w(x, v)+w(v, y) ;$

3. Se $(x, v),(v, y) \in A(D)$ e $(v, x),(y, v) \notin A(D)$ então

defina $D^{\prime}:=D /(v, y) ; w(x, v):=w(x, v)+w(v, y) ;$

4. Considere os casos simétricos de 2 e 3 , com os papéis de $x$ e $y$ trocados.

Devolva $\left(D^{\prime},\left.w\right|_{D^{\prime}}\right)$. 
Denote por $\gamma(D, w)$ o peso do circuito mínimo de tamanho ao menos 3 em $(D, w)$. Claramente, se $\left(D^{\prime}, w^{\prime}\right)$ foi obtido de $(D, w)$ por uma operação RAP então $\gamma(D, w)=$ $\gamma\left(D^{\prime}, w^{\prime}\right)$. Por outro lado, se $\left(D^{\prime}, w^{\prime}\right)$ vem de uma operação EVE então $\gamma(D, w)=$ $\min \left\{w(C), \gamma\left(D^{\prime}, w^{\prime}\right)\right\}$ onde $C$ é um circuito mínimo de tamanho 3 contendo $v, x$ e $y$ (se não existir tal circuito, defina $w(C)=+\infty)$.

Como $D$ é série-paralelo e não possui vértices de corte ele possui um vértice especial pelo Lema 2.6.1. Ainda, as operações RAP e EVE mantêm o grafo série-paralelo e não criam vértices de corte. Assim, um simples algoritmo polinomial que resolve $\operatorname{MC} \mathrm{P}(D, w)$ consiste de sucessivas aplicações dessas duas operações.

\section{Algoritmo MC3}

Entrada: Um grafo orientado série-paralelo $(D, w)$.

Saída: Um circuito mínimo em $(D, w)$ de comprimento ao menos 3 .

Defina $C:=\emptyset$ e $w(C):=+\infty$;

Enquanto $|V(D)| \geq 4$ faça

Enquanto $(D, w)$ possui arcos paralelos faça $(D, w):=\operatorname{RAP}(D, w)$;

Se $(D, w)$ possui um vértice especial $v$ então

Seja $C^{\prime}$ o circuito mínimo de comprimento 3 contendo $v$;

Se $w\left(C^{\prime}\right)<w(C)$ então $C:=C^{\prime}$;

$(D, w):=\operatorname{EVE}(D, w ; v)$;

Calcule um circuito $C^{\prime}$ de peso mínimo em $(D, w) ; \quad(*|V(D)| \leq 3 *)$

Se $w\left(C^{\prime}\right)<w(C)$ então $C:=C^{\prime}$;

Devolva o circuito correspondente a $C$ no grafo orientado original $(D, w)$.

O fato de que o Algoritmo MC3 está correto segue das observações acima. Isto completa a descrição de um algoritmo polinomial que encontra um circuito mínimo em $(M, w)$.

\subsection{Cobertura uniforme por circuitos}

Um caso especial do PCEC de bastante interesse e para o qual ainda existem vários problemas em aberto é aquele em que todos os pesos são iguais a um inteiro $k>0$. Dizemos que $M$ admite uma $k$-cobertura exata por circuitos se existe uma coleção de circuitos de $M$ tal que cada arco e cada aresta de $M$ pertence a exatamente $k$ circuitos dessa coleção. O problema de interesse é caracterizar os grafos que admitem uma $k$ cobertura exata por circuitos, para cada $k>0$.

Claramente, um grafo orientado $D$ admite uma $k$-cobertura exata por circuitos se, e somente se, $D$ é euleriano, para todo $k>0$. 
Para grafos não-orientados esse problema foi resolvido para todo $k \neq 2$. Se $k$ é ímpar, é fácil ver que $G$ admite uma $k$-cobertura exata por circuitos se, e somente se, $G$ é euleriano. Para $k$ par, uma condição necessária simples para que um grafo admita uma $k$-cobertura exata por circuitos é que $G$ não contenha pontes. Bermond, Jackson e Jaeger [15] provaram que todo grafo sem pontes admite uma 4-cobertura exata por circuitos, e Fan [30] mostrou que todo grafo sem pontes admite uma 6-cobertura exata por circuitos. Isto mostra que todo grafo sem pontes admite uma $2 k$-cobertura exata por circuitos para todo $k \geq 2$. O caso $k=2$ é um problema famoso e difícil.

Conjectura 2.7.1 (Conjectura da Cobertura Dupla por Circuitos [65, 67]). Todo grafo não-orientado sem pontes admite uma 2-cobertura exata por circuitos.

Sabe-se que essa conjectura é válida para várias classes de grafos: grafos livres de Petersen (Teorema 2.4.4) e grafos que admitem um 4-fluxo (Lema 2.4.2). Para grafos arbitrários, no entanto, o problema continua em aberto. O Teorema 2.4 .4 mostra ainda que um possível contra-exemplo dessa conjectura deve conter um menor do grafo de Petersen.

Para grafos mistos não se conhecem muito resultados. O caso $k=1$ corresponde a caracterizar grafos que podem ser decompostos em circuitos disjuntos nos arcos e nas arestas. Vimos no Capítulo 1 que um grafo misto $M$ admite uma tal decomposição se, e somente se é euleriano, isto é, se $f_{M}(X):=|\delta(X)|-|| \delta^{-}(X)|-| \delta^{+}(X)||$ é não-negativo e par para todo corte $\nabla(X)$. Em vista deste último resultado, é fácil ver que para $k$ ímpar, um grafo misto $M$ admite uma $k$-cobertura exata por circuitos se, e somente se, é euleriano.

Se $k$ for par, uma condição claramente necessária para que $M$ admita uma $k$-cobertura exata por circuitos é que $f_{M}(X) \geq 0$ para todo $X \subseteq V(M)$ e que $M$ não contenha pontes. O Teorema 2.5.2 mostra que essa condição é suficiente se $M$ é série-paralelo.

Teorema 2.7.1. Seja $M$ um grafo misto série-paralelo sem pontes. Então $M$ admite uma 2-cobertura exata por circuitos se, e somente se, $f_{M}(X) \geq 0$ para todo $X \subseteq V(M)$.

Podemos considerar várias questões relativas ao resultado acima. Dizemos que um grafo misto $M$ é admissível se não contém pontes e $f_{M}(X) \geq 0$ para todo $X \subseteq V(M)$.

1. É possível eliminar a hipótese de $M$ ser série-paralelo no teorema acima, isto é, todo grafo misto admissível admite uma 2-cobertura exata por circuitos? Observe, no entanto, que uma resposta afirmativa a esta questão implicaria a conjectura da cobertura dupla por circuitos.

2. Existe algum inteiro par $k$ tal que todo grafo misto admissível admite uma $k$ cobertura exata por circuitos?

3. É verdade que para cada grafo misto admissível $M$ existe uma constante $k_{M}$ tal que $M$ admite uma $k_{M}$-cobertura exata por circuitos? Ou equivalentemente, é 
verdade que se $M$ é admissível então $(M, 1)$ admite uma cobertura fracionária por circuitos?

Não temos respostas a nenhuma dessas perguntas mas observamos que esses problemas podem ser extremamente difíceis em vista do Teorema 2.5.1.

\subsection{Bases de Hilbert, subespaços lineares e reticulados} $H$.

Seja $H$ um subconjunto finito de $\mathbb{Q}^{n}$. Considere os seguintes conjuntos gerados por

$$
\begin{aligned}
\operatorname{Lin}(H) & =\left\{\sum_{h \in H} \lambda_{h} h \mid \lambda_{h} \in \mathbb{Q}\right\}, \\
\operatorname{Cone}(H) & =\left\{\sum_{h \in H} \lambda_{h} h \mid \lambda_{h} \in \mathbb{Q}_{+}\right\}, \\
\operatorname{Lat}(H) & =\left\{\sum_{h \in H} \lambda_{h} h \mid \lambda_{h} \in \mathbb{Z}\right\}, \\
\operatorname{Int} \operatorname{Cone}(H) & =\left\{\sum_{h \in H} \lambda_{h} h \mid \lambda_{h} \in \mathbb{Z}_{+}\right\} .
\end{aligned}
$$

Eles são chamados respectivamente de (sub)espaço linear, cone, reticulado ${ }^{3}$ e cone inteiro gerado por $H$. Os três últimos conjuntos podem ser relacionados através da seguinte inclusão: IntCone $(H) \subseteq \operatorname{Cone}(H) \cap \operatorname{Lat}(H)$. Entretanto, nem sempre vale a igualdade. Dizemos que $H$ é uma base de Hilbert se $\operatorname{Int} \operatorname{Cone}(H)=\operatorname{Cone}(H) \cap \operatorname{Lat}(H)$. $\mathrm{O}$ conceito de base de Hilbert aparece no contexto de vários problemas combinatórios e de programação inteira que não trataremos aqui. Propriedades de bases de Hilbert e sua relação com programação inteira podem ser encontradas em [63], enquanto em [64] há uma discussão dos vários problemas em aberto da área. Em [38], pode-se encontrar uma discussão sobre os quatro conjuntos acima nos casos em que $H$ é o conjunto dos (vetores de incidência de) circuitos ou o conjunto dos emparelhamentos perfeitos de um grafo não-orientado.

Nesta seção nos concentramos no caso em que $H$ é o conjunto dos (vetores de incidência de) circuitos de um grafo misto $M=(V, E, A)$. Denotamos os conjuntos acima simplesmente por $\operatorname{Lin}\left(\mathcal{C}_{M}\right), \operatorname{Cone}\left(\mathcal{C}_{M}\right), \operatorname{Lat}\left(\mathcal{C}_{M}\right)$ e Int Cone $\left(\mathcal{C}_{M}\right)$, respectivamente. Nesta terminologia, o PCFC é equivalente a perguntar se um dado vetor $p \in \mathbb{Q}^{E \cup A}$ pertence a Cone $\left(\mathcal{C}_{M}\right)$, e o PCEC é equivalente a perguntar se $p$ pertence a $\operatorname{IntCone}\left(\mathcal{C}_{M}\right)$.

Quando $M=D$ é um grafo orientado, pela Proposição 2.3.1 temos $\operatorname{IntCone}\left(\mathcal{C}_{D}\right)=$ Cone $\left(\mathcal{C}_{D}\right) \cap \operatorname{Lat}\left(\mathcal{C}_{D}\right)$, e portanto, o conjunto dos (vetores de incidência de) circuitos de um grafo orientado formam uma base de Hilbert.

\footnotetext{
${ }^{3}$ Em inglês, "lattice", daí a notação.
} 
Quando $M=G$ é um grafo não-orientado, vimos que o cone gerado pelos circuitos de $G$ consiste dos vetores $p \in \mathbb{Q}^{E(G)}$ que satisfazem as seguintes desigualdades:

$$
\begin{aligned}
p(\delta(X)-e)-p(e) \geq 0 & & \text { para todo } X \subseteq V, e \in \delta(X), \\
p(e) \geq 0 & & \text { para todo } e \in E(G) .
\end{aligned}
$$

Como todo vetor $p \in \operatorname{Cone}\left(\mathcal{C}_{G}\right) \cap \operatorname{Lat}\left(\mathcal{C}_{G}\right)$ é admissível, pelo Teorema 2.4.4 temos que os circuitos de um grafo não-orientado $G$ livre de Petersen formam uma base de Hilbert.

Para grafos mistos série-paralelos, temos um resultado análogo. O Corolário 2.5.1 afirma que se $M$ é série-paralelo então o cone gerado pelos circuitos de $M$ é completamente descrito pelas seguintes desigualdades:

$$
\begin{aligned}
& p(\delta(X))-\left|p\left(\delta^{-}(X)\right)-p\left(\delta^{+}(X)\right)\right| \geq 0 \quad \text { para todo } X \subseteq V, \\
& p(\nabla(X)-e)-p(e) \geq 0 \quad \text { para todo } X \subseteq V, e \in \delta(X), \\
& p(e) \geq 0 \quad \text { para todo } e \in E \cup A \text {. }
\end{aligned}
$$

Além disso, como todo vetor $p \in \operatorname{Cone}\left(\mathcal{C}_{M}\right) \cap \operatorname{Lat}\left(\mathcal{C}_{M}\right)$ é admissível, segue do Teorema 2.5.2 que os circuitos de um grafo misto série-paralelo $M$ formam uma base de Hilbert. Em vista do Teorema 2.5.1, é improvável que existam boas caracterizações completas de Cone $\left(\mathcal{C}_{M}\right)$ e de $\operatorname{IntCone}\left(\mathcal{C}_{M}\right)$ para grafos mistos arbitrários.

Um problema em aberto na teoria de bases de Hilbert é determinar um limite superior justo para o número máximo de elementos em uma base de Hilbert necessário para escrever qualquer vetor de IntCone $(H)$ como uma combinação linear inteira não-negativa de elementos de $H$. O seguinte resultado foi demonstrado por Sebő [64].

Teorema 2.8.1 (Sebő, 1990). Seja $H \subset \mathbb{Q}^{n}$ uma base de Hilbert. Então todo vetor de IntCone $(H)$ pode ser escrito como uma combinação linear inteira náa-negativa de no
máximo $2 n-2$ elementos de $H$.

Sebő [64] conjecturou que $2 n-2$ pode ser substituído por $n$ no teorema acima. O próximo resultado mostra que esta conjectura vale para bases de Hilbert formadas pelos circuitos em um grafo misto série-paralelo.

Teorema 2.8.2. Seja $M=(V, E, A)$ um grafo misto série-paralelo. Então todo vetor $p \in \operatorname{IntCone}\left(\mathcal{C}_{M}\right)$ pode ser escrito como uma combinação linear inteira não-negativa de no máximo $m=|E \cup A|$ vetores de incidência de circuitos.

Prova. Seja $M=(V, E, A)$ um grafo misto série-paralelo e $p \in \operatorname{IntCone}\left(\mathcal{C}_{M}\right)$. Então $p$ é inteiro, satisfaz $(2.3),(2.4),(2.5), \mathrm{e} \nabla(X)$ é par para todo $X \subseteq V$. Note que em $(2.3)$ e $(2.4)$ podemos supor que $\nabla(X)$ é um corte minimal; se este não for o caso então a desigualdade correspondente é uma combinação linear não-negativa de outras desigualdades do mesmo tipo (cada corte é uma união de cortes minimais).

Seja $F$ uma face minimal de $\operatorname{Cone}\left(\mathcal{C}_{M}\right)$ contendo $p$. Vamos proceder por indução em $\operatorname{dim}(F)$, a dimensão de $F$. Se $\operatorname{dim}(F)=0$, então $p=0$ e o resultado segue. 
Suponha que $\operatorname{dim}(F)>0$. Suponha por hipótese de indução que para toda face $F^{\prime}$ de Cone $\left(\mathcal{C}_{M}\right)$ tal que $\operatorname{dim}\left(F^{\prime}\right)<\operatorname{dim}(F)$ vale o seguinte: se $\left(M, p^{\prime}\right)$ é admissível então $p^{\prime}$ pode ser escrito como uma combinação linear inteira não-negativa de no máximo $\operatorname{dim}\left(F^{\prime}\right)$ circuitos.

Como $(M, p)$ é admissível, segue do Teorema 2.5.2 que existe um circuito $C$ de $M$ tal que $\left(M, p-\chi^{C}\right)$ é admissível. Seja $\alpha:=\max \left\{t \mid p-t \chi^{C} \in\right.$ Cone $\left.(\mathcal{C})\right\}$. Claramente, $\alpha \geq 1$. Vamos provar que $\alpha$ é um inteiro. Seja $p^{\prime}:=p-\alpha \chi^{C}$. Pela escolha de $\alpha$ existe uma desigualdade $a^{T} x \geq 0$ definindo uma face de Cone $\left(\mathcal{C}_{M}\right)$ tal que $a^{T} p^{\prime}=0 \mathrm{e}$ $a^{T}\left(p-\lambda \chi^{C}\right) \geq 0$ para todo $\lambda>\alpha$. Dizemos que tal desigualdade é justa.

Vamos analisar as três possibilidades para $a^{T} x \geq 0$.

Caso 1: $a^{T} x \geq 0$ é do tipo (2.3). Seja $\nabla(X)$ o corte minimal correspondente a esta desigualdade. Como esta desigualdade é justa, temos $C \cap \delta(X) \neq \emptyset$ (em caso contrário, $\left.f_{p^{\prime}}(X)=f_{p}(X)\right)$. Pelo Lema 2.5.1 temos que $f_{p^{\prime}}(X)=f_{p}(X)-2 \alpha=0$. Como $(M, p)$ é admissível, $f_{p}(X)$ é par e portanto $\alpha$ é inteiro.

Caso 2: $a^{T} x \geq 0$ é do tipo (2.4). Então existe um corte minimal $\nabla(X)$ e uma aresta $e$ no corte tal que $p^{\prime}(\nabla(X)-e)-p^{\prime}(e)=0$. Como esta desigualdade é justa, temos que $e \notin C$. Então, pelo Lema 2.5.1, $p^{\prime}(\nabla(X)-e)-p^{\prime}(e)=p(\nabla(X)-e)-2 \alpha-p(e)=0$. Como $(M, p)$ é admissível, $p(\nabla(X)-e)-p(e)$ é par e portanto $\alpha$ é inteiro.

Caso 3: $a^{T} x \geq 0$ é do tipo (2.5). Então existe um arco ou aresta $e$ de $C$ tal que $p^{\prime}(e)=p(e)-\alpha=0$. Como $p$ é inteiro segue que $\alpha$ é inteiro.

Isto mostra que $\alpha$ é um inteiro e que $\left(M, p^{\prime}\right)$ é admissível. Como $a^{T} x \geq 0$ é justa existe uma face minimal $F^{\prime} \subseteq F$ contendo $p^{\prime}$ tal que $\operatorname{dim}\left(F^{\prime}\right)<\operatorname{dim}(F)$. Pela hipótese de indução $p^{\prime}$ pode ser escrito como uma combinação linear inteira não-negativa de no máximo $\operatorname{dim}\left(F^{\prime}\right)$ circuitos. Como $p=p^{\prime}+\alpha \chi^{C}$, segue que $p$ pode ser escrito como uma combinação linear inteira não-negativa de no máximo $\operatorname{dim}(F)$ circuitos.

Embora o problema de caracterizar $\operatorname{Cone}\left(\mathcal{C}_{M}\right)$ seja difícil, a tarefa é mais fácil para $\operatorname{Lin}\left(\mathcal{C}_{M}\right)$ e $\operatorname{Lat}\left(\mathcal{C}_{M}\right)$. Descrevemos agora boas caracterizações do subespaço linear $\operatorname{Lin}\left(\mathcal{C}_{M}\right)$ e e do reticulado $\operatorname{Lat}\left(\mathcal{C}_{M}\right)$ gerado pelos (vetores de incidência de) circuitos de um grafo misto $M=(V, E, A)$.

Vejamos primeiro uma descrição do subespaço linear dos circuitos. Inicialmente, vamos supor que $M$ é fortemente conexo. Como veremos, é simples estender essa descrição para grafos mistos arbitrários.

No caso orientado ou não-orientado o subespaço linear possui uma caracterização simples. Se $M=D$ é um grafo orientado fortemente conexo então é fácil ver que

$$
\operatorname{Lin}\left(\mathcal{C}_{D}\right)=\left\{p \in \mathbb{Q}^{A(D)} \mid p\left(\delta^{+}(v)\right)=p\left(\delta^{-}(v)\right) \quad \text { para todo } v \in V(D)\right\} .
$$

Por outro lado, se $M=G$ é um grafo não-orientado então

$\operatorname{Lin}\left(\mathcal{C}_{G}\right)=\left\{p \in \mathbb{Q}^{E(G)} \mid p(e)=0\right.$ para toda ponte e e $p(a)=p(b)$ para todo corte $\left.\{a, b\}\right\}$. 
Para grafos mistos fortemente conexos, temos as seguintes condições necessárias que um vetor $p \in \operatorname{Lin}\left(\mathcal{C}_{M}\right)$ deve satisfazer:

$$
\begin{aligned}
p(e) & =0 & & \text { para toda ponte } e ; \\
p\left(e_{1}\right) & =p\left(e_{2}\right) & & \text { para todo corte }\left\{e_{1}, e_{2}\right\} \\
p\left(\delta^{+}(X)\right) & =p\left(\delta^{-}(X)\right) & & \text { para todo } X \subseteq V \text { tal que } \delta(X)=\emptyset ; \\
p\left(\delta^{+}(X)\right) & =p(\delta(X)) & & \text { para todo } X \subseteq V \text { tal que } \delta^{-}(X)=\emptyset \text { e }|\delta(X)|=1 .
\end{aligned}
$$

Mostraremos que as equações lineares acima formam uma descrição completa de $\operatorname{Lin}\left(\mathcal{C}_{M}\right)$.

Primeiro, observe que podemos supor que não existem pontes, e podemos eliminar cortes formados por exatamente duas arestas de modo similar ao feito na prova do Teorema 2.5.2. Além disso, se existir um corte $\nabla(X)$ tal que $\delta^{-}(X)=\emptyset$ e $\delta(X)=\{u v\}$ com $u \in X$, então podemos orientar $u v$ de $v$ a $u$ sem alterar o subespaço linear dos circuitos, ou seja, o subespaço linear do grafo resultante é isomorfo ao do grafo original. Portanto, daqui em diante suporemos que $M$ é reduzido, ou seja, não contém cortes deste tipo, pontes e nem cortes de arestas de tamanho 2 .

Vamos precisar do seguinte resultado, demonstrado por Boesch e Tindell [19].

Teorema 2.8.3. Um grafo misto fortemente conexo $M$ admite uma orientação fortemente conexa se, e somente se, não possui pontes.

Logo, todo grafo misto reduzido $M$ admite uma orientação fortemente conexa. Além disso, para toda aresta $e, M-e$ admite uma orientação fortemente conexa.

Suponha que $M=D \cup G$. Considere a matriz $B:=B_{M}$ com linhas indexadas por subconjuntos $X$ de $V(M)$ tais que $\delta(X)=\emptyset$ e com colunas indexadas pelos arcos e arestas de $M$, definida como:

$$
B[X, a]= \begin{cases}+1 & \text { se } a \in \delta^{+}(X), \\ -1 & \text { se } a \in \delta^{-}(X), \\ 0 & \text { caso contrário. }\end{cases}
$$

Assim, queremos mostrar que $\operatorname{Lin}\left(\mathcal{C}_{M}\right)=\{p \mid B p=0\}$, ou seja, o subespaço linear gerado pelos circuitos de $M$ é exatamente o complemento ortogonal do espaço linha de $B$. Vamos denotar por $c(G)$ o número de componentes de $G$.

Lema 2.8.1. O posto de B é igual a $c(G)-1$.

Prova. Qualquer coluna indexada por uma aresta ou um arco com ambos os extremos em um mesmo componente de $G$ é um vetor nulo. Assim, podemos removê-las de $B$. Sejam $X_{1}, \ldots, X_{t}$ os (conjuntos de vértices dos) componentes de $G$. É fácil ver que qualquer linha de $B$ é uma combinação linear das linhas correspondentes a esses componentes. Podemos remover todas as linhas que não correspondem a algum dos $X_{i}$ 's. Esta matriz corresponde à matriz de incidência do grafo orientado $D^{\prime}$ obtido de $M$ contraindo cada $X_{i}$ a um vértice. É um fato bem conhecido que o posto desta matriz é igual a $\left|V\left(D^{\prime}\right)\right|-1$ e portanto, o posto de $B$ é é igual a $c(G)-1$. 
Teorema 2.8.4. Se $M=(V, E, A)$ é um grafo misto reduzido então $\operatorname{Lin}\left(\mathcal{C}_{M}\right)=\{p \in$ $\left.\mathbb{Q}^{E \cup A} \mid B p=\mathbf{0}\right\}$.

Prova. Claramente, $\operatorname{Lin}\left(\mathcal{C}_{M}\right) \subseteq\{p \mid B p=0\}$. Vamos provar a outra inclusão. Pelo Lema 2.8.1 é suficiente exibir $m-c(G)+1$ vetores linearmente independentes de $\operatorname{Lin}\left(\mathcal{C}_{M}\right)$, onde $m=|E \cup A|$.

Seja $D^{\prime}=M / E$. Note que $D^{\prime}:=\left(V^{\prime}, A^{\prime}\right)$ é um grafo orientado fortemente conexo. É um fato conhecido que $\operatorname{dim}\left(\operatorname{Lin}\left(\mathcal{C}_{D^{\prime}}\right)\right)=\left|A^{\prime}\right|-\left|V^{\prime}\right|+1=|A|-c(G)+1$ e portanto, $D^{\prime}$ contém $d:=|A|-c(G)+1$ circuitos linearmente independentes $C_{1}, \ldots, C_{d}$. É fácil ver que tais circuitos podem ser estendidos a circuitos linearmente independentes de $M$ acrescentando algumas arestas a esses.

Agora mostraremos que $\chi^{e} \in \operatorname{Lin}\left(\mathcal{C}_{M}\right)$ para todo $e \in E$. Seja $e=x y$ uma aresta de $E$. O grafo $M-e$ admite uma orientação fortemente conexa $D^{\prime \prime}$, e portanto, existem um caminho orientado $P_{1}$ de $x$ a $y$ em $D^{\prime \prime}$ e um caminho orientado $P_{2}$ de $y$ a $x$ em $D^{\prime \prime}$. Logo,

$$
2 \chi^{e}=\chi^{P_{1}+e}+\chi^{P_{2}+e}-\left(\chi^{P_{1}}+\chi^{P_{2}}\right) .
$$

É fácil ver que $\chi^{P_{1}}+\chi^{P_{2}}$ pode ser escrito como uma soma de circuitos de $D^{\prime \prime}$ (e portanto de $M$ ). Logo, $\chi^{e} \in \operatorname{Lin}\left(\mathcal{C}_{M}\right)$.

Juntando esses $|E|$ vetores unitários linearmente independentes aos $|A|-c(G)+1$ circuitos obtidos previamente, obtemos $m-c(G)+1$ vetores linearmente independentes de $\operatorname{Lin}\left(\mathcal{C}_{M}\right)$. Isto completa a prova.

Corolário 2.8.1. Seja $M=(V, E, A)$ um grafo misto. Então $p \in \operatorname{Lin}\left(\mathcal{C}_{M}\right)$ se, e somente se, $p$ satisfaz as seguintes restrições:

$$
\begin{aligned}
p(a) & =0 & & \text { para todo } a \text { pertencente a um dicorte; } \\
p(e) & =0 & & \text { para toda ponte } e ; \\
p\left(e_{1}\right) & =p\left(e_{2}\right) & & \text { para todo corte }\left\{e_{1}, e_{2}\right\} ; \\
p\left(\delta^{+}(X)\right) & =p\left(\delta^{-}(X)\right) & & \text { para todo } X \subseteq V \text { tal que } \delta(X)=\emptyset ; \\
p\left(\delta^{+}(X)\right) & =p(\delta(X)) & & \text { para todo } X \subseteq V \text { tal que } \delta^{-}(X)=\emptyset \text { e }|\delta(X)|=1 .
\end{aligned}
$$

Agora vamos voltar ao estudo do reticulado $\operatorname{Lat}\left(\mathcal{C}_{M}\right)$ gerado pelos circuitos de um grafo misto $M=(V, E, A)$. Como seria razoável esperar, se $M=D$ for um grafo orientado, a descrição do reticulado é muito simples:

$$
\operatorname{Lat}\left(\mathcal{C}_{D}\right)=\operatorname{Lin}\left(\mathcal{C}_{D}\right) \cap \mathbb{Z}^{A} .
$$

Se $M=G$ é um grafo não-orientado, temos

$$
\operatorname{Lat}\left(\mathcal{C}_{G}\right)=\operatorname{Lin}\left(\mathcal{C}_{G}\right) \cap\left\{p \in \mathbb{Z}^{E}: p(\delta(X)) \text { é par para todo } X \subseteq V\right\} .
$$

Para grafos mistos, temos o seguinte resultado que generaliza os dois acima. 
Teorema 2.8.5. Seja $M=(V, E, A)$ um grafo misto. Então

$$
\operatorname{Lat}\left(\mathcal{C}_{M}\right)=\operatorname{Lin}\left(\mathcal{C}_{M}\right) \cap\left\{p \in \mathbb{Z}^{E \cup A} \mid p(\nabla(X)) \text { é par para todo } X \subseteq V\right\} .
$$

Prova. Claramente, $\operatorname{Lat}\left(\mathcal{C}_{M}\right) \subseteq \operatorname{Lin}\left(\mathcal{C}_{M}\right) \cap\{p \mid p(\nabla(X))$ é par para todo $X \subseteq V\}$.

Para provar a outra inclusão, podemos supor que $M$ é fortemente conexo e reduzido. Seja $p$ um vetor inteiro de $\operatorname{Lin}\left(\mathcal{C}_{M}\right)$ tal que $p(\nabla(X))$ é par para todo corte $\nabla(X)$. Vamos provar que $p \in \operatorname{Lat}\left(\mathcal{C}_{M}\right)$.

Denote $A_{p \neq 0}:=\{a \in A \mid p(a) \neq 0\}$. Repita o seguinte procedimento sobre o grafo misto $H:=\left(V, E, A_{p \neq 0}\right)$ :

1. Se $A_{p \neq 0}=\emptyset$ então pare.

2. Escolha um arco $a \in A_{p \neq 0}$.

3. Encontre um circuito $C$ em $H:=\left(V, E, A_{p \neq 0}\right)$ contendo $a$.

4. Faça $p:=p-p(a) \chi^{C}$. Vá para o Passo 1 .

Note que no Passo 3 o circuito $C$ sempre pode ser encontrado já que em cada iteração $H$ não possui dicortes, pois $p \in \operatorname{Lin}\left(\mathcal{C}_{M}\right)$ (isso é verdade na primeira iteração e a operação executada no Passo 4 não altera esse fato).

Então após no máximo $|A|$ iterações obtemos um vetor $p \in \operatorname{Lin}\left(\mathcal{C}_{M}\right)$ com suporte contido em $E$. Note que a operação executada no Passo 4 também não altera a paridade de nenhum corte. Como no Teorema 2.8.4, podemos provar que $2 \chi^{e} \in \operatorname{Lat}\left(\mathcal{C}_{M}\right)$ para toda aresta $e \in E$. Portanto, podemos supor que $p$ é um vetor com entradas iguais a 0 ou 1. Logo, o suporte de $p$ corresponde a um ciclo, e portanto, $p$ pode ser escrito como uma soma de circuitos. Isto completa a prova.

Outra questão interessante que não exploramos neste trabalho é o de encontrar bases de reticulados de circuitos. Uma base de um reticulado $L a t(H)$ é um conjunto $S$ de vetores linearmente independentes tal que todo vetor de $L a t(H)$ pode ser escrito como uma combinação linear inteira de vetores de $S$. É um fato conhecido que se $H$ é formado de vetores racionais então $\operatorname{Lat}(H)$ contém uma base [63] $]^{4}$ Galluccio e Loebl [36] provaram que tanto o reticulado dos circuitos de um grafo orientado como o de um grafo nãoorientado contêm uma base formada apenas por circuitos. O problema mais geral de determinar se $\operatorname{Lat}\left(\mathcal{C}_{M}\right)$ contém uma base formada apenas por circuitos de $M$ continua em aberto.

\subsection{Conclusão}

Apresentamos nesta seção duas tabelas com os principais resultados vistos neste capítulo sobre o PCEC e o PCFC. Embora o PCEC e o PCFC estejam formulados como

\footnotetext{
${ }^{4}$ Observe no entanto que não é necessariamente verdade que existe uma base contida em $H$. Por exemplo, $\mathbb{Z}$ é gerado por $\{2,3\}$ mas $\{2,3\}$ não contém nenhuma base de $\mathbb{Z}$.
} 
problemas de decisão, na coluna de Complexidade de cada tabela quando escrevemos "Polinomial" queremos dizer que o problema de busca correspondente pode ser resolvido em tempo polinomial. A única exceção é o PCEC restrito a grafos não-orientados livre de Petersen, para o qual não se conhece um algoritmo polinomial de busca, mas apenas para o de decisão.

Incluímos também nestas tabelas os resultados sobre a complexidade computacional do PCEC e do PCFC restrito a grafos mistos planares que são demonstrados no próximo capítulo. Outros resultados sobre o PCEC e o PCFC serão vistos no Capítulo 5. Lá discutimos a complexidade desses problemas em grafos com largura arbórea limitada.

\begin{tabular}{||c|c|c|c||}
\hline \hline \multicolumn{4}{|c||}{ PCEC } \\
\hline \multirow{4}{*}{ Orientado } & Complexidade & Referência \\
\hline \multirow{4}{*}{ Não-orientado } & $\begin{array}{c}\text { 4-aresta-conexo } \\
p(e)=1 \text { ou } 2\end{array}$ & Polinomial & {$[42]$} \\
\cline { 2 - 5 } & Planar & Polinomial & {$[45,44]$} \\
\cline { 2 - 5 } & Livre de Petersen & Polinomial & {$[2]$} \\
& & $\begin{array}{c}\text { (sabe-se apenas resolver } \\
\text { o problema de decisão) }\end{array}$ & \\
\cline { 2 - 5 } & Arbitrário & Em aberto & - \\
\hline \multirow{6}{*}{ Misto } & Série-Paralelo & Polinomial & Seção 2.6 \\
\cline { 2 - 5 } & Planar & NP-difícil & Capítulo 3 \\
\cline { 2 - 5 } & Arbitrário & NP-difícil & {$[10,8]$} \\
\hline \hline
\end{tabular}

Tabela 2.1: Tabela dos principais resultados sobre o PCEC. 


\begin{tabular}{|c|c|c|c|}
\hline \multicolumn{4}{|c|}{ PCFC } \\
\hline \multicolumn{2}{|c|}{ Grafo } & Complexidade & Referência \\
\hline \multicolumn{2}{|c|}{ Orientado } & Polinomial & {$[42]$} \\
\hline \multicolumn{2}{|c|}{ Não-orientado } & Polinomial & {$[65,10]$} \\
\hline \multirow{3}{*}{ Misto } & Série-Paralelo & Polinomial & Seção 2.6 \\
\hline & Planar & NP-completo & Capítulo 3 \\
\hline & Arbitrário & NP-completo & {$[10,8]$} \\
\hline
\end{tabular}

Tabela 2.2: Tabela dos principais resultados sobre o PCFC. 


\section{Cobertura exata por circuitos em grafos mistos planares}

\subsection{Introdução}

Mencionamos no Capítulo 2 que o PCEC e o PCFC são problemas NP-completos [10, 8]. Descrevemos também um algoritmo polinomial para o PCEC/PCFC em grafos mistos série-paralelos.

Em vista dos resultados obtidos para essa classe, surgiu de maneira natural o interesse em tentar estender esses resultados para a classe dos grafos planares. Além disso, esta classe parece ser bastante natural para o estudo de problemas combinatórios envolvendo circuitos e/ou caminhos. De fato, algumas vezes é possível aproveitar a imersão no plano ou outra propriedade característica de grafos planares para desenvolver algoritmos polinomiais para o problema em questão. Por exemplo, o problema de decidir se um grafo não-orientado planar contém um circuito induzido de comprimento ímpar (respectivamente, par) pode ser resolvido em tempo polinomial ([43],[55]).

Entretanto, veremos que o PCEC e o PCFC continuam difíceis mesmo para a classe de grafos planares. Na próxima seção provamos que o PCEC Planar e o PCFC Planar (os correspondentes problemas restritos a grafos mistos planares) são NP-completos.

\subsection{Complexidade do PCEC/PCFC Planar}

O problema NP-completo que usaremos para reduzir ao PCEC (PCFC) Planar é um caso particular do 3-SAT: o 3-SAT Planar [53]. Uma instância do 3-SAT usual, denotada por $F(C, X)$, consiste de uma fórmula booleana $F$ com cláusulas $C=\left\{C_{1}, \ldots, C_{m}\right\}$ e variáveis $X=\left\{v_{1}, \ldots, v_{n}\right\}$, e o problema consiste em decidir se existe uma atribuição de valores às variáveis que satisfaz a fórmula $F$. Para definir o 3-SAT Planar considere o 
grafo não-orientado $G(F):=\left(C \cup X, E_{1} \cup E_{2}\right)$ onde

$$
\begin{aligned}
& E_{1}=\left\{C_{i} v_{j}: v_{j} \in C_{i} \text { ou } \bar{v}_{j} \in C_{i}\right\} \mathrm{e} \\
& E_{2}=\left\{v_{j} v_{j+1}: 1 \leq j<n\right\} \cup\left\{v_{n} v_{1}\right\} .
\end{aligned}
$$

Note que a estrutura de $G(F)$ depende da indexação das variáveis. O 3-SAT Planar corresponde ao problema 3-SAT restrito a fórmulas $F(C, X)$ para as quais existe (e é dada) uma indexação das variáveis tal que $G(F)$ é planar.

Antes de prosseguirmos, é conveniente fazermos uma observação: como foi mencionado no capítulo anterior, dada uma instância $(M, p)$ do PCEC (que admite uma cobertura por circuitos), não é óbvio que existe uma cobertura com um número polinomial de circuitos se os pesos dos arcos/arestas forem muito grandes, ou em linguagem de complexidade computacional, não é óbvio que o PCEC pertence a NP. Na redução que fazemos os pesos são pequenos (1 e 2) e nesse caso particular, o PCEC pertence a NP.

Descrevemos agora a redução do 3-SAT Planar ao PCEC Planar. No final do capítulo mostramos que a mesma funciona para o PCFC Planar.

Suponha que $F(C, X)$ seja uma instância de 3-SAT Planar e fixe uma imersão plana de $G(F)$. Podemos supor que cada cláusula em $F$ contém pelo menos duas variáveis. Na Figura 3.1(i) temos um exemplo de grafo $G(F)$.

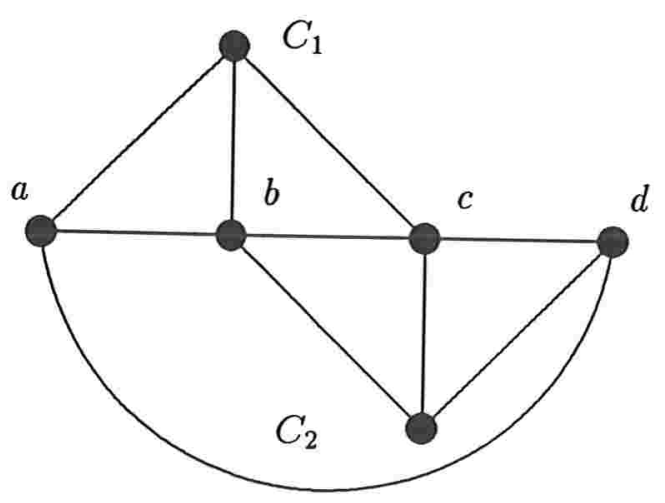

(i)



(ii)

Figura 3.1: (i) Grafo $G(F)$ da fórmula $F=(a+\bar{b}+c)(b+c+\bar{d})$. (ii) Grafo $\vec{G}(F)$.

Considere o grafo orientado $\vec{G}(F)$ obtido de $G(F)$ da seguinte forma. Orientamos as arestas de $E_{2}$ de modo a obter o circuito $\left(v_{1}, v_{2}, \ldots, v_{n}, v_{1}\right)$; depois substituímos cada aresta da forma $v_{j} C_{i}$ por um 2-circuito orientado. A orientação no plano de cada 2circuito depende da sua posição em relação ao circuito orientado $\left(v_{1}, v_{2}, \ldots, v_{n}, v_{1}\right)$. Se o vértice $C_{i}$ estiver na região externa em relação a esse circuito, então o circuito contendo $v_{j}$ e $C_{i}$ é orientado no sentido horário, caso contrário, é orientado no sentido anti-horário (veja Figura 3.1(ii)). 
A partir de $\vec{G}(F)$ construímos um grafo misto $(M, p)$ substituindo os vértices de $\vec{G}(F)$ (variáveis e cláusulas) por certos "super-vértices" (grafos) que descrevemos a seguir. Nesse grafo cada aresta tem peso 2 e cada arco tem peso 1.

Substituímos cada variável $v_{j}$ por um grafo $L\left(v_{j}\right)$ como na Figura 3.2, identificando cada arco $\left(v_{j}, v_{j+1}\right)$ de $\vec{G}(F)$ com um arco $\left(t_{j}, s_{j+1}\right)$, para $j=1, \ldots, n$ onde $s_{n+1}=s_{1}$.

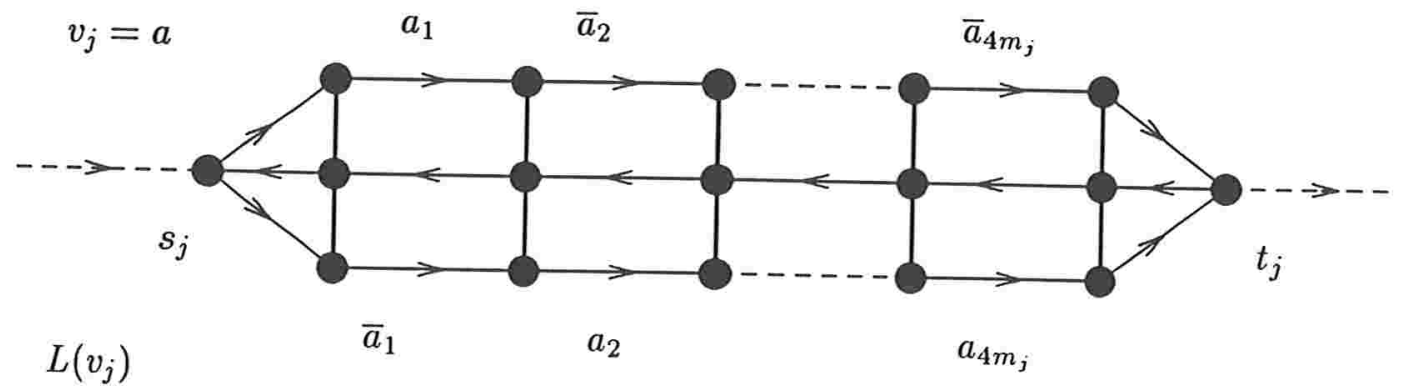

Figura 3.2: Grafo $L\left(v_{j}\right)$ correspondente à variável $v_{j}$.

No grafo $L\left(v_{j}\right)$ o número $m_{j}$ indica quantas vezes a variável $v_{j}$ (negada ou não) aparece na fórmula $F$. O número $4 m_{j}$ é escolhido de modo a ser suficientemente grande (em geral, pode-se escolher um número menor) para permitir intervalos entre seções verticais de $L\left(v_{j}\right)$ ligando diferentes cláusulas. A necessidade disto ficará clara adiante.

Descrevemos agora os super-vértices correspondentes às cláusulas. Suponha que $C_{i}$ seja uma cláusula com três literais. Então ela é substituída por um grafo $H\left(C_{i}\right)$ como na Figura 3.3(i), onde os três vértices destacados $h_{1}, h_{2}, h_{3}$ são identificados com as variáveis da cláusula $C_{i}$. Note que em relação à estrutura de $H\left(C_{i}\right)$ existe uma simetria entre $h_{2}$ e $h_{3}$ mas $h_{1}$ é um vértice diferenciado. Para $t=2,3$ dizemos que a aresta $e_{t}$ é a aresta oposta a $h_{t}$ e que $h_{1}$ é o vértice livre de $H\left(C_{i}\right)$. Se $C_{i}$ é uma cláusula com apenas dois literais então ela é substituída por um grafo $H\left(C_{i}\right)$ como na Figura 3.3(ii). Note que os arcos incidentes aos vértices $h_{t}$ representam os arcos incidentes às variáveis de $C_{i}$ no grafo $\vec{G}(F)$.

Resta-nos descrever como as cláusulas são ligadas às variáveis. Suponha que $C_{i}$ seja uma cláusula contendo um literal $v_{j}$. Ligamos $H\left(C_{i}\right)$ a $L\left(v_{j}\right)$ da seguinte forma. Suponha que $v_{j}$ esteja identificado com o super-vértice $h_{t}$ (que corresponde ao grafo $L\left(v_{j}\right)$ ). Escolha um arco $a_{k}=(x, y)$ em $L\left(v_{j}\right)\left(a=v_{j}\right)$, remova-o e faça o arco que sai de $h_{t}$ entrar em $y$ e o arco que entra em $h_{t}$ sair de $x$. A escolha de $a_{k}$ é indiferente mas deve haver intervalos entre ligações de $H\left(C_{i}\right)$ 's distintos e a substituição deve ser feita de modo a manter a planaridade do grafo resultante. Não é difícil ver que isso é sempre possível uma vez que $4 m_{j}$ é suficientemente grande. No caso de $C_{i}$ conter um literal $\bar{a}_{j}$, escolhe-se um arco $\bar{a}_{k}=(x, y)$ (em vez de $a_{k}$ ) para ser removido e liga-se $x, y$ a $H\left(C_{i}\right)$ como antes. Note que os dois arcos que ligam $H\left(C_{i}\right)$ a $a_{k}$ (ou $\bar{a}_{k}$ ) representam o 2-circuito $\left(v_{j}, C_{i}, v_{j}\right)$ de $\vec{G}(F)$. 
(i)
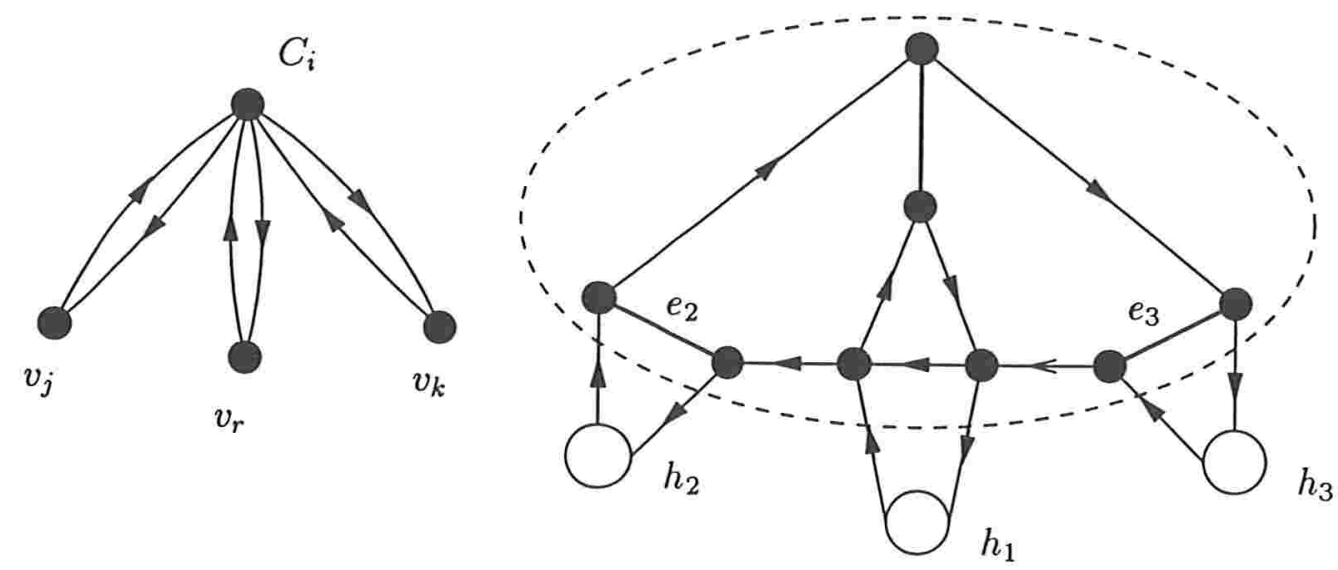

$H\left(C_{i}\right)$

(ii)
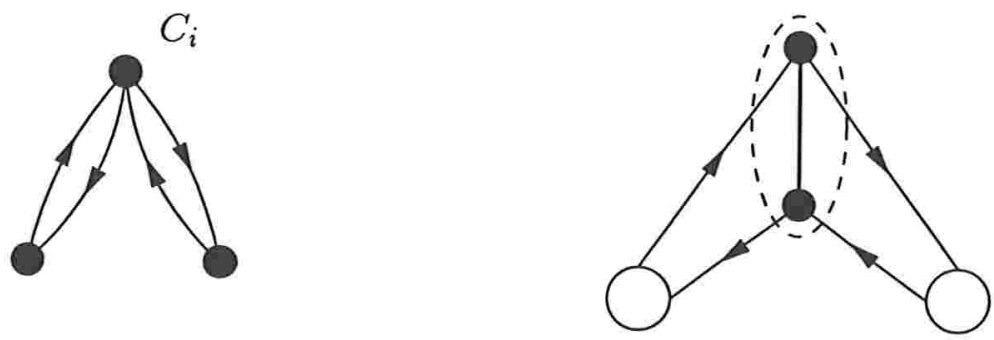

$H\left(C_{i}\right)$

Figura 3.3: (i) Grafo $H\left(C_{i}\right)$ correspondente a uma cláusula $C_{i}$ contendo 3 literais. (ii) Grafo $H\left(C_{i}\right)$ correspondente a uma cláusula $C_{i}$ contendo 2 literais.

Com isso, concluímos a construção do grafo misto plano $(M, p)$ a partir de $G(F)$. Veja um exemplo na Figura 3.4. Note que nesse exemplo usamos $2 m_{j}$ como "comprimento" de cada $L\left(v_{j}\right)$ em vez de $4 m_{j}$. 





Mostraremos que se $(M, p)$ admite uma cobertura exata por circuitos então existe um circuito (com determinadas características) que passa por todos os $\operatorname{arcos}\left(t_{j}, s_{j+1}\right)$, para $i=1, \ldots, n$. Antes disso, faremos algumas observações sobre os grafos $L\left(v_{j}\right)$ e $H\left(C_{i}\right)$.

O grafo $L\left(v_{j}\right)$ acrescentado de um arco $\left(t_{j}, s_{j}\right)$ admite uma cobertura exata por circuitos. Existem duas possibilidades para o circuito $C$ que contém $\left(t_{j}, s_{j}\right): C-\left(t_{j}, s_{j}\right)$ é um caminho em ziguezague que vai de $s_{j}$ a $t_{j}$ que contém todos os arcos $a_{k}$, para $k=1, \ldots, 4 m_{j}$ (veja Figura 3.5 ), ou então $C-\left(t_{j}, s_{j}\right)$ é um caminho em ziguezague que vai de $s_{j}$ a $t_{j}$ que contém todos os arcos $\bar{a}_{k}$, para $k=1, \ldots, 4 m_{j}$. Note que os demais arcos e arestas de $L\left(v_{j}\right)$ podem ser cobertos por circuitos locais.

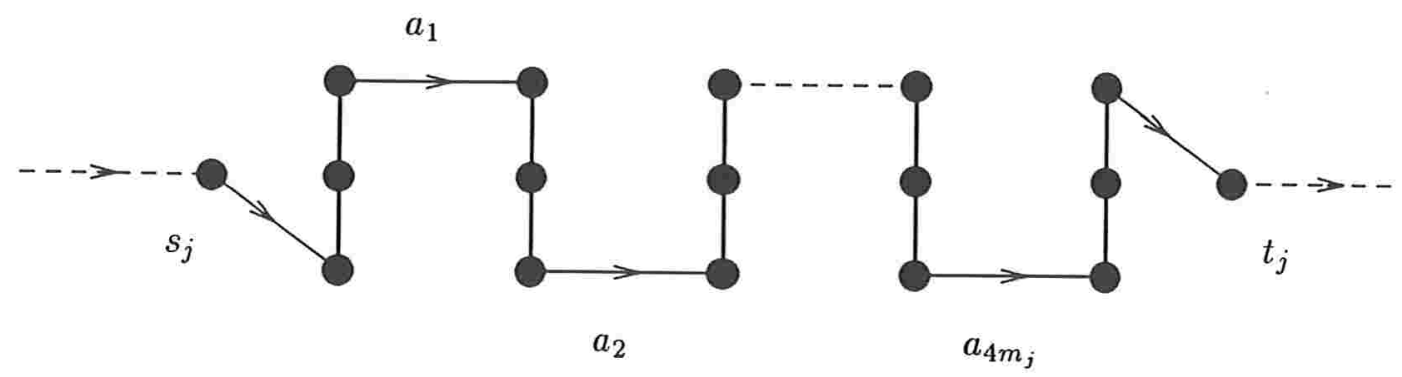

Figura 3.5: Caminho em ziguezague.

O grafo $H\left(C_{i}\right)$ (pensando nos $h_{j}$ 's como vértices) admite uma cobertura exata por circuitos. Ela é única no caso de $C_{i}$ conter apenas dois literais. Vamos discutir o caso no qual $C_{i}$ contém três literais. Neste caso, uma cobertura de $H\left(C_{i}\right)$ consiste de exatamente quatro circuitos. Dois desses são necessariamente os dois triângulos contendo $h_{2}$ e $h_{3}$ e suas respectivas arestas opostas. Existem exatamente duas possibilidades para os outros dois circuitos (veja a Figura 3.6). Note que o circuito que contém $h_{1}$ deve conter ou $e_{2}$ ou $e_{3}$.

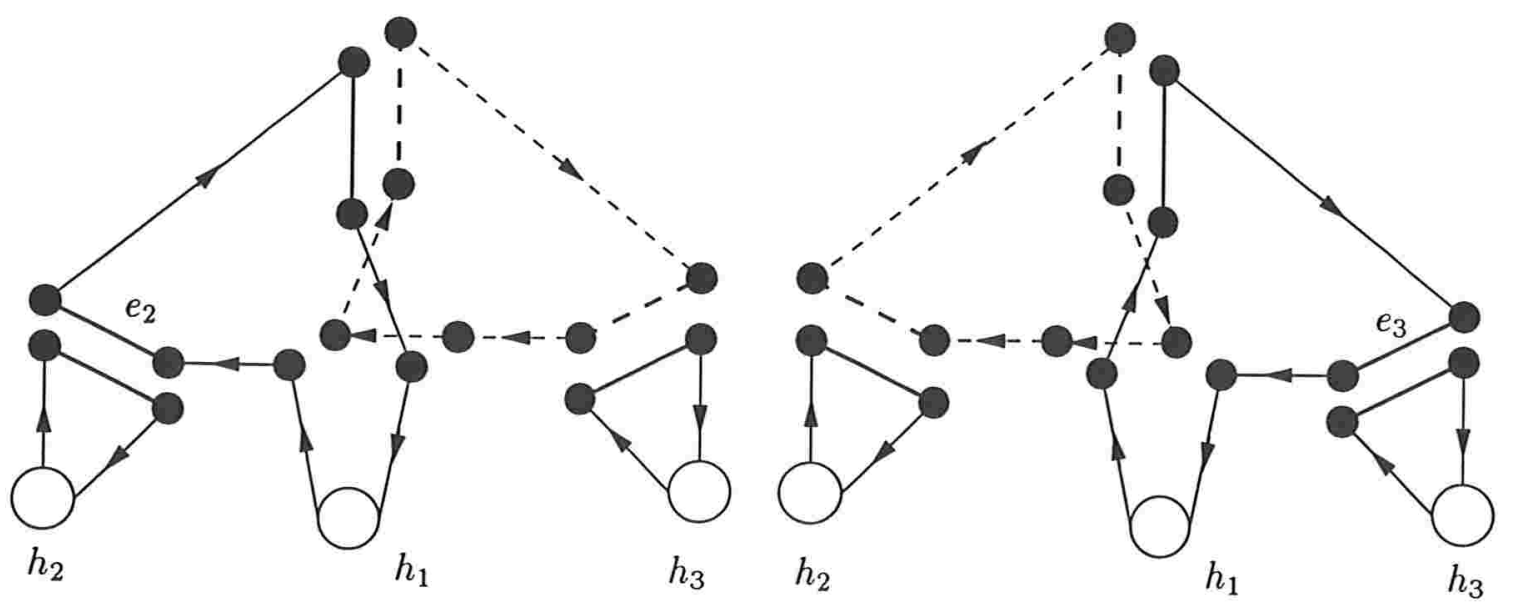

Figura 3.6: Possíveis circuitos na cobertura de $H\left(C_{i}\right)$. 
Os dois triângulos contendo $h_{2}$ e $h_{3}$ juntamente com os dois possíveis circuitos contendo $h_{1}$ induzem caminhos em $H\left(C_{i}\right)$ (pensando agora em $H\left(C_{i}\right)$ como um subgrafo de $M)$. Chamamos tais caminhos de cadeias. Analogamente, existem duas cadeias quando $C_{i}$ contém apenas duas cláusulas. Assim, a cada cadeia de $H\left(C_{i}\right)$ está associado um literal da cláusula $C_{i}$, e para cada literal da cláusula $\left(v_{r}\right.$ ou $\left.\bar{v}_{r}\right)$ existe uma cadeia correspondente. No caso em que a variável $v_{r}$ foi identificada com o vértice livre $h_{1}$ existem duas cadeias candidatas.

Agora é fácil ver que se $(M, p)$ admite uma cobertura exata por circuitos então um circuito $C$ dessa cobertura deve conter todos os arcos $\left(t_{j}, s_{j+1}\right)$, para $i=1, \ldots, n$. De fato, se $C$ entra em uma variável $L\left(v_{j}\right)$ pelo arco $\left(t_{j-1}, s_{j}\right)$ então ele deve usar um dos dois caminhos em ziguezague e sair por $\left(t_{j}, s_{j+1}\right)$. Eventualmente, no caminho em ziguezague ele pode sair da variável $L\left(v_{j}\right)$ e entrar em uma cláusula $H\left(C_{i}\right)$, mas dentro desta o circuito é forçado a usar uma cadeia e retornar à variável $L\left(v_{j}\right)$.

Dizemos que um circuito $C$ em $M$ é global se este passa por todos os arcos $\left(t_{j}, s_{j+1}\right)$, para $i=1, \ldots, n$, e passa em cada um dos subgrafos $L\left(v_{j}\right)$ sempre usando um dos caminhos em ziguezague (eventualmente entrando numa cláusula $H\left(C_{i}\right)$, se um arco $a_{k}$ foi removido, percorrendo uma das cadeias correspondentes a $v_{j}$ e voltando a $L\left(v_{j}\right)$ ).

Agora estamos em condições de provar que o PCEC e o PCFC são NP-completos. Vejamos primeiro a demonstração para o PCEC. Esta consiste dos dois seguintes lemas.

Lema 3.2.1. O grafo $(M, p)$ admite uma cobertura exata por circuitos se e somente se existe um circuito global em $M$.

Prova. Como vimos acima, se $(M, p)$ admite uma cobertura exata por circuitos então existe um circuito global em $M$.

Por outro lado, se $M$ contém um circuito global $C$ então é fácil ver que pode-se obter uma cobertura exata por circuitos para $(M, p)$ usando $C$ e circuitos locais a cada variável $L\left(v_{j}\right)$ e a cada cláusula $H\left(C_{i}\right)$.

Lema 3.2.2. O grafo $M$ contém um circuito global se e somente se a fórmula $F$ pode ser satisfeita.

Prova. Suponha que $M$ contenha um circuito global $C$. Note que $C$ não pode usar três cadeias de $H\left(C_{i}\right)$ (se $C_{i}$ contém apenas dois literais então $C$ só pode usar uma das cadeias) e portanto, para cada cláusula $C_{i}$ existe um literal contido nela cuja cadeia correspondente não é usada por $C$. Atribuindo valor verdadeiro a esses literais obtemos uma atribuição de valores que satisfaz $F$. Note que essa escolha é consistente pois ao percorrer um dos caminhos em ziguezague, ou seja, um dos literais de uma variável $L\left(v_{j}\right)$ o circuito $C$ é forçado a passar por todas as cláusulas $H\left(C_{i}\right)$ que contêm esse literal usando uma cadeia correspondente.

Se $F$ possui uma atribuição de valores que a satisfaz, então podemos definir um circuito global $C$ como segue. Percorremos $M$ através do "circuito" $\left(v_{1}, \ldots, v_{n}, v_{1}\right)$ de 
modo apropriado em cada $L\left(v_{j}\right)$ : se $v_{j}\left(\bar{v}_{j}\right)$ é falso, escolhemos o caminho em ziguezague que usa os arcos $a_{k}\left(\bar{a}_{k}\right)$ para $k=1, \ldots, 4 m_{j}$. Se este caminho entrar em uma cláusula $H\left(C_{i}\right)$ percorremos a cadeia correspondente a $v_{j}$. Se $v_{j}$ não foi identificado com o vértice livre, então existe apenas uma escolha. Senão, $C_{i}$ possui três literais e um deles torna a cláusula verdadeira. Percorremos então a cadeia correspondente a $v_{j}$ que contém a aresta oposta a esse literal verdadeiro (note que como o literal satisfaz a cláusula o circuito não poderá entrar novamente na cláusula através da variável correspondente). Isso define um circuito global $C$.

Dos lemas 3.2.1 e 3.2.2 podemos concluir o seguinte.

Teorema 3.2.1. O PCEC Planar é NP-completo mesmo com pesos restritos a 1 e 2 .

Finalmente, resta-nos verificar que o PCFC Planar também é NP-completo. Para isso, é suficiente mostrar que o Lema 3.2.1 continua válido se substituirmos "cobertura exata por circuitos" por "cobertura fracionária por circuitos".

Lema 3.2.3. O grafo $(M, p)$ admite uma cobertura fracionária por circuitos se e somente se existe um circuito global em $M$.

Prova. Se $M$ contém um circuito global $C$, então podemos obter uma cobertura exata por circuitos de $(M, p)$ como na demonstração do Lema 3.2.1.

Por outro lado, suponha que $\lambda_{1}, \ldots, \lambda_{t}$ e $C_{1}, \ldots, C_{t}$ formem uma cobertura fracionária por circuitos de $(M, p)$. Primeiramente, note que se $v$ é um vértice de $(M, p)$ de grau 3 onde incide exatamente uma aresta (de peso 2) e dois arcos (um entrando em $v$ e outro saindo de $v$ ), então nenhum circuito $C_{i}$ pode usar os dois arcos incidentes a $v$, pois senão a aresta incidente a $v$ ficaria com um excedente positivo que não poderia ser totalmente coberto por eventuais circuitos. Portanto, não é difícil ver que um circuito $C_{i}$ que contém o arco $\left(t_{n}, s_{1}\right)$ deve ser um circuito global.

Dos Lemas 3.2.2 e 3.2.3 segue o resultado desejado.

Teorema 3.2.2. O PCFC Planar é NP-completo mesmo com pesos restritos a 1 e 2 . 


\section{O problema do caminho mínimo e do circuito negativo}

\subsection{Introdução}

Neste capítulo abordamos um caso particular dos seguintes problemas.

\section{Problema do Circuito Negativo (PCN)}

Dado um grafo misto $M=(V, E, A)$ com uma função custo $c: E \cup A \mapsto \mathbb{Q}$, encontre um circuito (de custo) negativo, se existir.

\section{Problema do Caminho Mínimo (PCM)}

Dado um grafo misto $M=(V, E, A)$, uma função custo $c: E \cup A \mapsto \mathbb{Q}$ e vértices $s, t$ de $M$, encontre um caminho mínimo de $s$ a $t$ (suponha que exista um caminho de $s$ a $t$ ).

As principais razões para o estudo do PCN neste trabalho, além do fato deste ser um problema por si mesmo interessante, foram a observação das implicações algorítmicas para o Problema da Cobertura Fracionária por Circuitos (PCFC) e a tentativa de estabelecer a complexidade computacional do PCFC para grafos mistos planares (feito no capítulo anterior). Na Seção 4.2 mostramos que se o PCN pode ser resolvido em tempo polinomial para uma certa classe de grafos, então o PCFC também pode ser resolvido em tempo polinomial para grafos dessa classe.

O PCN aparece em vários outros contextos mas provavelmente é mais conhecido por estar relacionado ao problema de encontrar caminhos mínimos. é um fato bem conhecido que o PCM é NP-difícil se permitirmos circuitos negativos no grafo (isto pode ser visto por uma simples redução do problema do circuito hamiltoniano). Em vista disso, é natural considerar o PCN e o PCM para grafos $(M, c)$ conservativos, isto é, que não possuem circuitos negativos. 
Quando $M$ é um grafo orientado ou um grafo não-orientado o PCN e o PCM para grafos conservativos podem ser resolvidos em tempo polinomial $([23,16,34,68,69,50])$.

Entretanto, como foi mostrado por Arkin e Papadimitriou [9, 8], tanto o PCN quanto o PCM para grafos mistos conservativos são em geral problemas NP-difíceis. Em vista deste resultado torna-se interessante a busca por classes especiais de grafos para os quais o PCN e/ou o PCM possam ser resolvidos em tempo polinomial.

No Capítulo 2 vimos um algoritmo polinomial para encontrar um circuito mínimo em um grafo misto série-paralelo $(M, c)$ não necessariamente conservativo. Esse algoritmo pode ser ligeiramente modificado para resolver o PCM. Portanto, tanto o PCN quanto o PCM podem ser resolvidos em tempo polinomial em grafos mistos série-paralelos.

$\mathrm{Na}$ Seção 4.3 mostramos que o PCN e o PCM permanecem NP-difíceis para grafos mistos planares. Chamamos as versões de decisão desses problemas restritos a grafos planares de PCN Planar e PCM Planar.

\subsection{PCN versus PCFC}

Nesta seção mostramos como o PCN está relacionado com o PCFC e as implicações algorítmicas para este último problema.

Suponha que $\mathcal{M}$ seja uma classe de grafos mistos para os quais o PCN pode ser resolvido em tempo polinomial. Nosso objetivo é mostrar que se $M$ é um grafo arbitrário em $\mathcal{M}$, então o PCFC pode ser resolvido em tempo polinomial para $M$.

Seja $M$ um grafo em $\mathcal{M}$ e seja $N$ a matriz de incidência dos circuitos de $M$ (as colunas de $N$ consistem dos vetores característicos dos circuitos de $M)$. Suponha que $(M, p)$ seja uma instância do PCFC. Claramente, $\operatorname{PCFC}$ para $(M, p)$ corresponde ao problema de encontrar um vetor racional $\lambda \geq 0$ que satisfaz o sistema $N \lambda=p$. Isto é equivalente ao problema de encontrar uma solução ótima do seguinte PL:

$$
\max \{\lambda \cdot 0 \mid N \lambda=p, \lambda \geq 0\} .
$$

Formulando o PCFC desta maneira, temos associado a (4.1) o seu programa linear dual:

$$
\min \left\{p x \mid N^{T} x \geq 0\right\} \text {. }
$$

O problema da separação (veja o Capítulo 1) para (4.2) é o seguinte: dado um vetor racional $y$, ou certifique que y satisfaz $N^{T} y \geq 0$, ou encontre uma desigualdade violada.

Em nosso caso, uma desigualdade violada corresponde à existência de um circuito negativo no grafo $(M, x)$. Pelo Teorema 1.2.1 do Capítulo 1 , se o problema da separação para (4.2) pode ser resolvido em tempo polinomial no tamanho de $M$ e de $p$, então podese encontrar soluções básicas ótimas de (4.1) e (4.2) em tempo polinomial no tamanho de $M$ e de $p$ através do método dos elipsóides.

Portanto, se o PCN pode ser resolvido em tempo polinomial para uma classe $\mathcal{M}$ de grafos, então o PCFC também pode ser resolvido em tempo polinomial para grafos dessa 
classe. Como vimos no último capítulo, o PCFC Planar é NP-completo para grafos mistos planares, e portanto, segue da discussão acima que o PCN Planar é NP-completo para grafos mistos planares.

Na próxima seção apresentamos uma demonstração "direta" desse fato, reduzindo o 3-SAT Planar ao PCN Planar. Uma das vantagens de proceder desta maneira é podermos mostrar que o PCN Planar é NP-completo mesmo com custos "pequenos"e grau limitado. Além disto, modificando essa prova mostramos que o PCM Planar é NP-completo.

\subsection{A complexidade do PCN/PCM Planar}

A demonstração de que o PCN Planar é NP-completo é bastante similar à feita para o PCEC Planar e o leitor que compreendeu aquela não terá nenhuma dificuldade para acompanhar a que faremos agora. No final da seção mostramos uma simples modificação da demonstração com a qual concluímos que o PCM Planar também é NP-completo.

Usamos como problema de partida novamente o 3-SAT Planar. Suponha que $F(C, X)$ seja uma instância do 3 -SAT Planar, onde $C=\left\{C_{1}, \ldots, C_{m}\right\}$ é o conjunto das cláusulas e $X=\left\{v_{1}, \ldots, v_{n}\right\}$ é o conjunto das variáveis da fórmula $F$. Podemos supor que cada cláusula em $F$ contém ao menos duas variáveis.

Fixe uma imersão plana do grafo $G(F)$ e construa a versão orientada plana $\vec{G}(F)$. Construímos a partir de $\vec{G}(F)$ um grafo misto plano $(M, c)$ substituindo cada vértice de $\vec{G}(F)$ por "super-vértices" apropriados que descrevemos a seguir.

Substituímos cada variável $v_{j}$ por um grafo $L\left(v_{j}\right)$ como na Figura 4.1, identificando cada arco $\left(v_{j}, v_{j+1}\right)$ de $\vec{G}(F)$ com um arco $\left(t_{j}, s_{j+1}\right)$, para $j=1, \ldots, n$ onde $s_{n+1}=s_{1}$. As arestas de $L\left(v_{j}\right)$ têm peso -1 e os arcos têm peso 1 com exceção dos que entram em $t_{j}$ que têm peso zero.

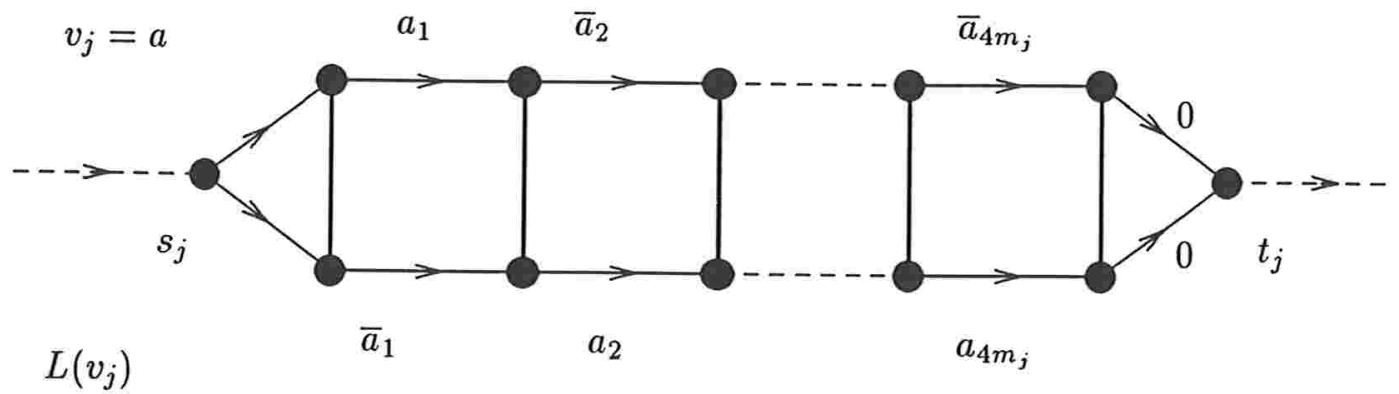

Figura 4.1: Grafo $L\left(v_{j}\right)$ correspondente à variável $v_{j}$.

Descreveremos agora os super-vértices correspondentes às cláusulas. Se a cláusula $C_{i}$ contém três literais então ela é substituída por um grafo $H\left(C_{i}\right)$ como na Figura 4.2(i), onde os três vértices destacados $h_{1}, h_{2}, h_{3}$ são identificados com as variáveis da cláusula $C_{i}$. Note que em relação à estrutura de $H\left(C_{i}\right)$ existe uma simetria entre $h_{2}$ e $h_{3}$ mas 
$h_{1}$ é um vértice diferenciado. Dizemos que $h_{1}$ é o vértice livre de $H\left(C_{i}\right)$. Se $C_{i}$ contém apenas dois literais então substituímo-la por um grafo $H\left(C_{i}\right)$ como na Figura 4.2(ii). As arestas de $H\left(C_{i}\right)$ têm peso -1 e os arcos têm peso 1 com exceção daqueles indicados na Figura 4.2 que têm peso zero.

(i)
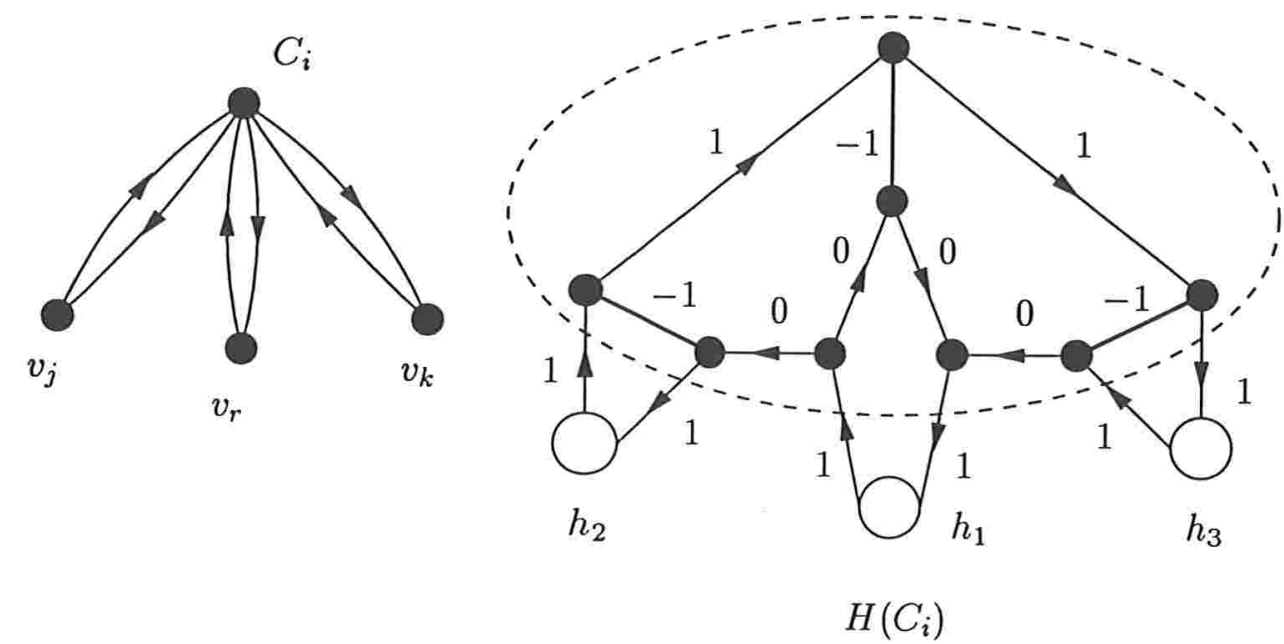

(ii)
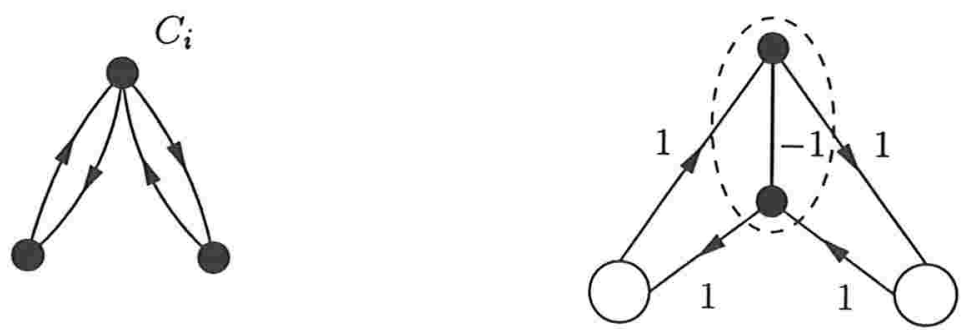

$H\left(C_{i}\right)$

Figura 4.2: (i) Grafo $H\left(C_{i}\right)$ correspondente a uma cláusula $C_{i}$ contendo 3 literais. (ii) Grafo $H\left(C_{i}\right)$ correspondente a uma cláusula $C_{i}$ contendo 2 literais.

Resta-nos descrever como as cláusulas são ligadas às variáveis. Suponha que $C_{i}$ seja uma cláusula contendo um literal $v_{j}$. Ligamos $H\left(C_{i}\right)$ a $L\left(v_{j}\right)$ da seguinte forma. Suponha que $v_{j}$ esteja identificado com o super-vértice $h_{t}$ (que corresponde ao grafo $L\left(v_{j}\right)$ ). Escolha um arco $a_{k}=(x, y)$ em $L\left(v_{j}\right)\left(a=v_{j}\right)$, remova-o e faça o arco que sai de $h_{t}$ entrar em $y$ e o arco que entra em $h_{t}$ sair de $x$. A escolha de $a_{k}$ é indiferente mas deve haver intervalos entre ligações de $H\left(C_{i}\right)$ 's distintos e a substituição deve ser feita de modo a manter a planaridade do grafo resultante. No caso de $C_{i}$ conter um literal $\bar{a}_{j}$, escolhe-se um arco $\bar{a}_{k}=(x, y)$ (em vez de $a_{k}$ ) para ser removido e liga-se $x, y$ a $H\left(C_{i}\right)$ como antes. Cada um dos dois novos arcos tem peso 1.

Finalmente, cada arco $\left(t_{j}, s_{j+1}\right)$ tem peso zero para $j=1, \ldots, n-1$, enquanto o arco $\left(t_{n}, s_{1}\right)$ tem peso -1 . Com isso, concluímos a construção do grafo misto plano $(M, c)$ a partir de $G(F)$. Veja um exemplo na Figura 4.3. 


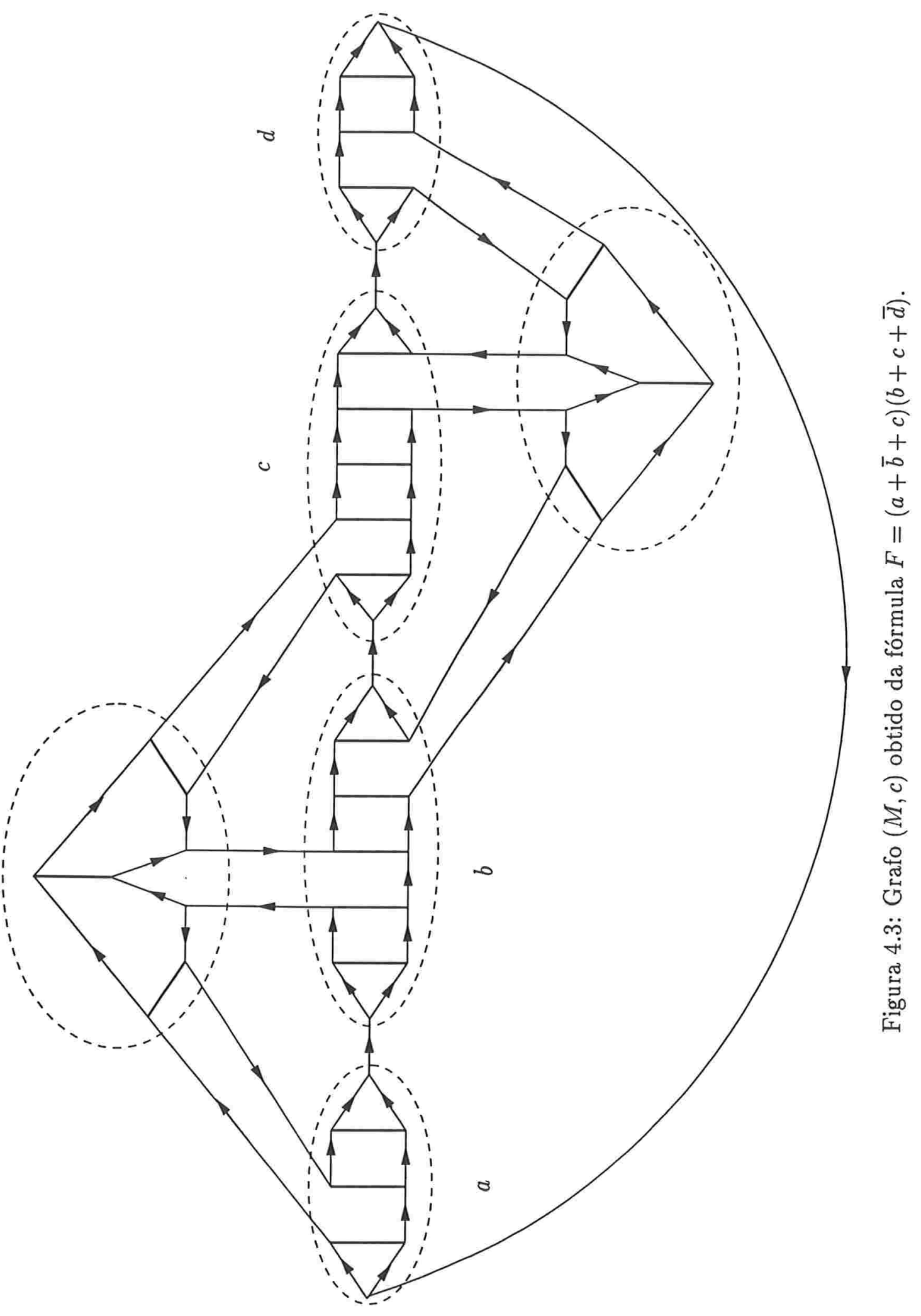


No grafo $L\left(v_{j}\right)$ existem exatamente dois caminhos de $s_{j}$ a $t_{j}$ de peso zero. Um deles é o caminho em ziguezague que contém todos os arcos $a_{k}$, para $k=1, \ldots, 4 m_{j}$ (veja Figura 4.4) e o outro é o caminho em ziguezague que contém todos os arcos $\bar{a}_{k}$, para $k=1, \ldots, 4 m_{j}$.

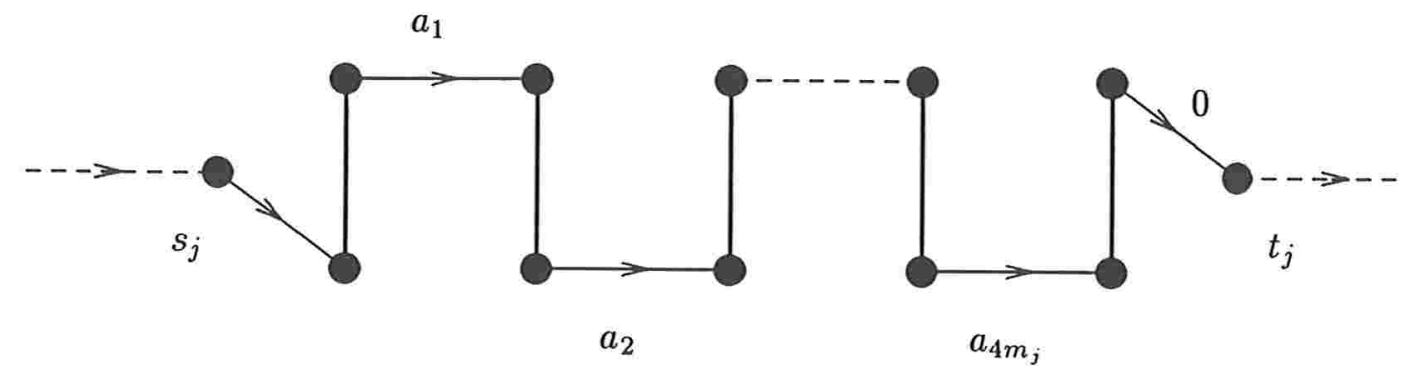

Figura 4.4: Caminho em ziguezague.

O grafo $H\left(C_{i}\right)$ (pensando nos $h_{j}$ 's como vértices) não possui circuitos de peso menor que 1. Além disso, existem exatamente quatro circuitos de peso 1. Dois deles são os triângulos contendo $h_{2}$ e $h_{3}$, e os outros dois circuitos que contêm $h_{1}$ estão indicados na Figura 4.5. Finalmente, note que qualquer caminho de um vértice $h_{i}$ a outro vértice $h_{j}$ tem peso estritamente maior que 1 .
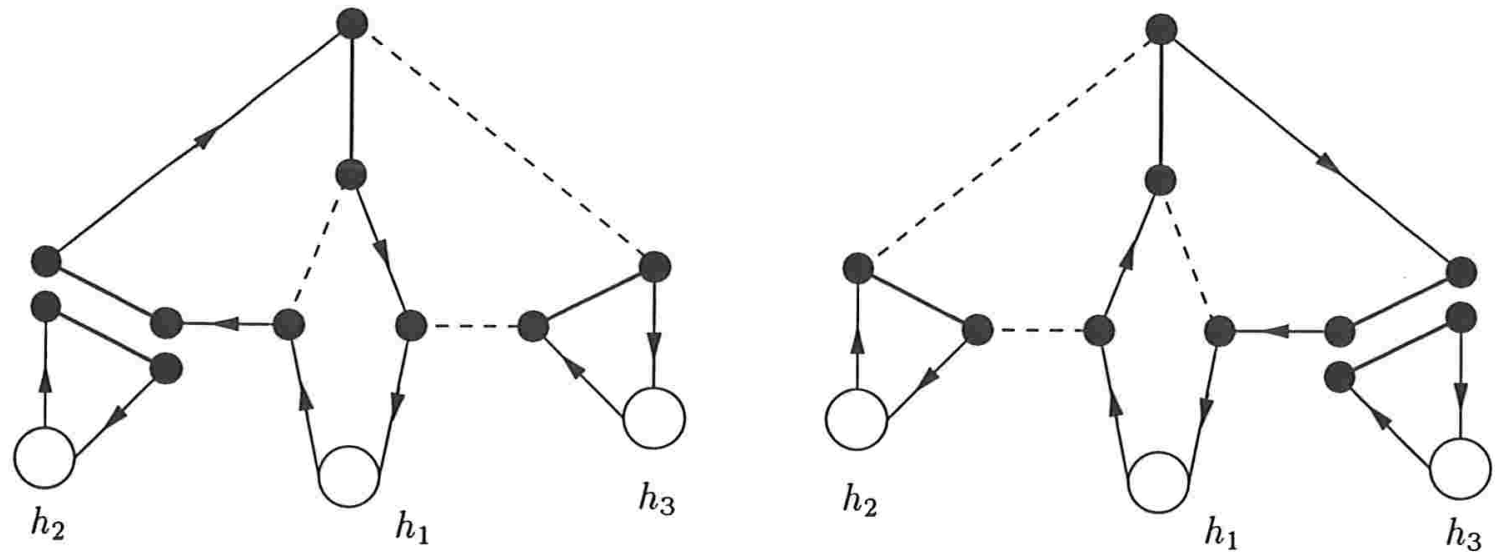

Figura 4.5: Possíveis circuitos de peso $1 \mathrm{em} H\left(C_{i}\right)$.

Os dois triângulos contendo $h_{2}$ e $h_{3}$ juntamente com os dois possíveis circuitos contendo $h_{1}$ induzem caminhos em $H\left(C_{i}\right)$ (pensando agora em $H\left(C_{i}\right)$ como um subgrafo de $M)$. Chamamos tais caminhos de cadeias. Analogamente, existem duas cadeias quando $C_{i}$ contém apenas duas cláusulas. Assim, a cada cadeia de $H\left(C_{i}\right)$ está associado um literal da cláusula $C_{i}$, e para cada literal da cláusula $\left(v_{r}\right.$ ou $\left.\bar{v}_{r}\right)$ existe uma cadeia correspondente. No caso em que a variável $v_{r}$ foi identificada com o vértice livre $h_{1}$ existem duas cadeias candidatas. 
Agora é fácil ver que se $(M, c)$ possui um circuito negativo $C$ então este circuito deve conter todos os arcos $\left(t_{j}, s_{j+1}\right)$, para $i=1, \ldots, n$ (lembre-se que o peso do arco $\left(t_{n}, s_{1}\right)$ é $-1)$. De fato, se $C$ entra em uma variável $L\left(v_{j}\right)$ pelo arco $\left(t_{j-1}, s_{j}\right)$ então ele deve usar um dos dois caminhos em ziguezague e sair por $\left(t_{j}, s_{j+1}\right)$. Eventualmente, no caminho em ziguezague ele pode sair da variável $L\left(v_{j}\right)$ e entrar em uma cláusula $H\left(C_{i}\right)$, mas dentro desta o circuito é forçado a usar uma cadeia e retornar à variável $L\left(v_{j}\right)$ (caso contrário a seção deste circuito ganharia um excesso positivo que não pode ser recuperado em outras partes do grafo $(M, c))$.

Vamos provar agora o resultado principal. Este segue diretamente do seguinte lema.

Lema 4.3.1. O grafo $(M, c)$ possui um circuito negativo se e somente se a fórmula $F$ pode ser satisfeita.

Prova. Suponha que $(M, c)$ possua um circuito negativo $C$. Note que $C$ não pode usar três cadeias de $H\left(C_{i}\right)$ e portanto, para cada cláusula $C_{i}$ existe um literal contido nela cuja cadeia correspondente não é usada por $C$. Atribuindo valor verdadeiro a esses literais obtemos uma atribuição de valores que satisfaz $F$. Note que essa escolha é consistente pois ao percorrer um dos caminhos em ziguezague, ou seja, um dos literais de uma variável $L\left(v_{j}\right)$, o circuito $C$ é forçado a passar por todas as cláusulas $H\left(C_{i}\right)$ que contêm esse literal usando uma cadeia correspondente.

Se $F$ possui uma atribuição de valores que a satisfaz, então podemos encontrar um circuito negativo $C$ como segue. Percorremos $M$ através do "circuito" $\left(v_{1}, \ldots, v_{n}, v_{1}\right)$ de modo apropriado em cada $L\left(v_{j}\right)$ : se $v_{j}\left(\bar{v}_{j}\right)$ é falso, escolhemos o caminho em ziguezague que usa os arcos $a_{k}\left(\bar{a}_{k}\right)$ para $k=1, \ldots, 4 m_{j}$. Se este caminho entrar em uma cláusula $H\left(C_{i}\right)$ percorremos a cadeia correspondente a $v_{j}$. Se $v_{j}$ não foi identificado com o vértice livre existe apenas uma escolha. Senão, $C_{i}$ possui três literais e um deles torna a cláusula verdadeira. Percorremos então a cadeia correspondente a $v_{j}$ que contém a aresta que forma em $H\left(C_{i}\right)$ um triângulo com esse literal verdadeiro. Isso define um circuito negativo $C$.

Teorema 4.3.1. O PCN Planar é NP-completo mesmo com pesos restritos a 0,1 e-1.

Para verificar que PCM Planar é NP-completo considere o mesmo grafo $(M, c)$ construído acima. Considere o grafo $M^{\prime}$ obtido a partir de $M$ removendo-se o arco $\left(t_{n}, s_{1}\right)$. Claramente $\left(M^{\prime}, c\right)$ não possui circuitos negativos. Além disso, $\left(M^{\prime}, c\right)$ possui um caminho de $s_{1}$ a $t_{n}$ de peso menor ou igual a zero (de fato, igual a zero) se e somente se a fórmula $F$ pode ser satisfeita. Obtemos então o resultado desejado.

Teorema 4.3.2. O PCM Planar é NP-completo mesmo com pesos restritos a 0,1 e -1 .

Nas Tabelas 4.1 e 4.2 apresentamos um resumo dos principais resultados conhecidos sobre o PCN e o PCM que foram discutidos neste capítulo. 


\begin{tabular}{|c|c|c|c|}
\hline \multicolumn{4}{|c|}{$\mathrm{PCN}$} \\
\hline \multicolumn{2}{|c|}{ Grafo } & Complexidade & Referência \\
\hline \multicolumn{2}{|c|}{ Orientado } & Polinomial & {$[23,16,50]$} \\
\hline \multicolumn{2}{|c|}{ Não-orientado } & Polinomial & {$[34,68,69,50]$} \\
\hline \multirow{3}{*}{ Misto } & Série-Paralelo & Polinomial & Capítulo 2 \\
\hline & Planar & NP-completo & Seção 4.3 \\
\hline & Arbitrário & NP-completo & {$[9,8]$} \\
\hline
\end{tabular}

Tabela 4.1: Tabela dos principais resultados sobre o PCN.

\begin{tabular}{|c|c|c|c|}
\hline \multicolumn{4}{|c|}{ PCM } \\
\hline \multicolumn{2}{|c|}{ Grafo } & Complexidade & Referência \\
\hline \multicolumn{2}{|c|}{ Orientado } & Polinomial & {$[23,16,50]$} \\
\hline \multicolumn{2}{|c|}{ Não-orientado } & Polinomial & {$[34,68,69,50]$} \\
\hline \multirow{3}{*}{ Misto } & Série-Paralelo & Polinomial & - \\
\hline & Planar & NP-completo & Seção 4.3 \\
\hline & Arbitrário & NP-completo & {$[9,8]$} \\
\hline
\end{tabular}

Tabela 4.2: Tabela dos principais resultados sobre o PCN. 


\section{O problema da cobertura mínima por circuitos}

\subsection{Introdução}

Neste capítulo estudamos outro tipo de problema de cobertura de grafos por circuitos. Uma cobertura por circuitos de um grafo misto $M$ é uma família $\mathcal{C}:=\left\{C_{1}, \ldots, C_{k}\right\}$ de circuitos que cobre todos os arcos e arestas de $M$, ou seja, cada arco/aresta de $M$ pertence a pelo menos um circuito dessa família. Definimos o peso da cobertura $\mathcal{C}$ como $\sum_{i=1}^{k}\left|C_{i}\right|$. Estamos interessados no seguinte problema.

\section{Problema da Cobertura Mínima por Circuitos (PCMC)}

Dado um grafo misto fortemente conexo sem pontes $M$, encontre uma cobertura por circuitos de $M$ de peso mínimo.

Este problema tem sido bastante estudado recentemente, principalmente no caso em que $M$ é um grafo não-orientado. Como veremos, ele está relacionado com o Problema da Cobertura Exata por Circuitos (PCEC), o Problema do Carteiro Chinês (PCC) e a conjectura da cobertura dupla por circuitos.

$\mathrm{Na}$ Seção 5.2 apresentamos a demonstração de Thomassen [70] de que o PCMC para grafos não-orientados arbitrários é NP-difícil. Usando o fato de que o PCEC é NPcompleto para grafos mistos planares, como demonstramos no Capítulo 3, apresentamos uma prova simples de que o PCMC é NP-difícil para grafos mistos planares.

$\mathrm{Na}$ Seção 5.3 estudamos alguns casos para os quais o PCMC pode ser resolvido em tempo polinomial ou para os quais existem evidências disso. Em todos esses casos o Problema do Carteiro Chinês (PCC) desempenha um papel crucial. Para grafos orientados, o PCC e o PCMC são polinomialmente equivalentes. Mostraremos, usando alguns resultados do Capítulo 2 sobre o PCEC que, para grafos não-orientados que não contêm um menor de Petersen, o valor ótimo desses dois problemas coincidem. No entanto, veremos 
que isto não implica imediatamente em um algoritmo polinomial para o PCMC nessa classe de grafos. Uma igualdade análoga é descrita para grafos mistos série-paralelos nesse caso, entretanto, existe um algoritmo polinomial para o PCMC que descrevemos na seção seguinte.

Na Seção 5.4 consideramos grafos mistos que possuem um certo parâmetro, a largura arbórea, limitado por uma constante. A largura arbórea de um grafo conexo mede de certa forma o quanto ele é semelhante a uma árvore. Árvores têm largura arbórea $1 \mathrm{e}$ grafos série-paralelos são os grafos com largura arbórea no máximo 2. Muitos problemas de otimização, inclusive alguns estudados neste trabalho, podem ser resolvidos em tempo polinomial em grafos (mistos) com largura arbórea limitada. Descrevemos, com detalhes, algoritmos polinomiais para o PCC e o PCMC.

Na Seção 5.5 estudamos algoritmos de aproximação para o PCMC. Apresentamos uma demonstração bastante engenhosa de Bermond, Jackson e Jaeger [15] do seguinte fato: todo grafo não-orientado sem pontes $G$ possui uma cobertura por circuitos de peso no máximo $5 / 3|E(G)|$. Veremos ainda que tal cobertura pode ser encontrada em tempo polinomial, e como $|E(G)|$ é um limite inferior óbvio para o peso de uma cobertura mínima por circuitos de $G$, isto implica em um algoritmo de $5 / 3$-aproximação para o PCMC em grafos não-orientados.

Antes de prosseguirmos convém observar que podemos considerar uma versão com pesos do PCMC onde, além do grafo misto $M=(V, E, A)$, é dada uma função peso $w$ : $E \cup A \mapsto \mathbb{Z}_{+}$e neste caso definimos o peso de uma cobertura $\mathcal{C}$ como sendo $\sum_{C \in \mathcal{C}} w(C)$. Assim, o PCMC constitui um caso particular deste problema em que $w(e)=1$ para todo $e \in E \cup A$. Todos os resultados que descrevemos neste capítulo também são válidos para essa versão com pesos.

\subsection{Complexidade do PCMC}

Nesta seção apresentamos a prova de Thomassen [70] de que o PCMC para grafos não-orientados é NP-difícil. Considere o problema de decisão correspondente ao PCMC em grafos não-orientados.

\section{PCMC-G}

Entrada: Um grafo não-orientado $G$ sem pontes e um inteiro $k$.

Questão: Existe uma cobertura de circuitos de $G$ com peso no máximo $k$ ?

Mostraremos então que o PCMC-G é NP-completo.

Considere o grafo $H$ indicado na Figura 5.1. Note que o grafo obtido a partir de $H$ removendo-se os vértices $x_{1}, x_{2}, x_{3}, y_{1}, y_{2}$ e $y_{3}$, e identificando os vértices $z_{1}, z_{2}, z_{3}$ é o
grafo de Petersen.

Denote por $H(t, q)$ (com $t \geq 1, q \geq 1$ ) o grafo obtido a partir de $H$ substituindo cada 


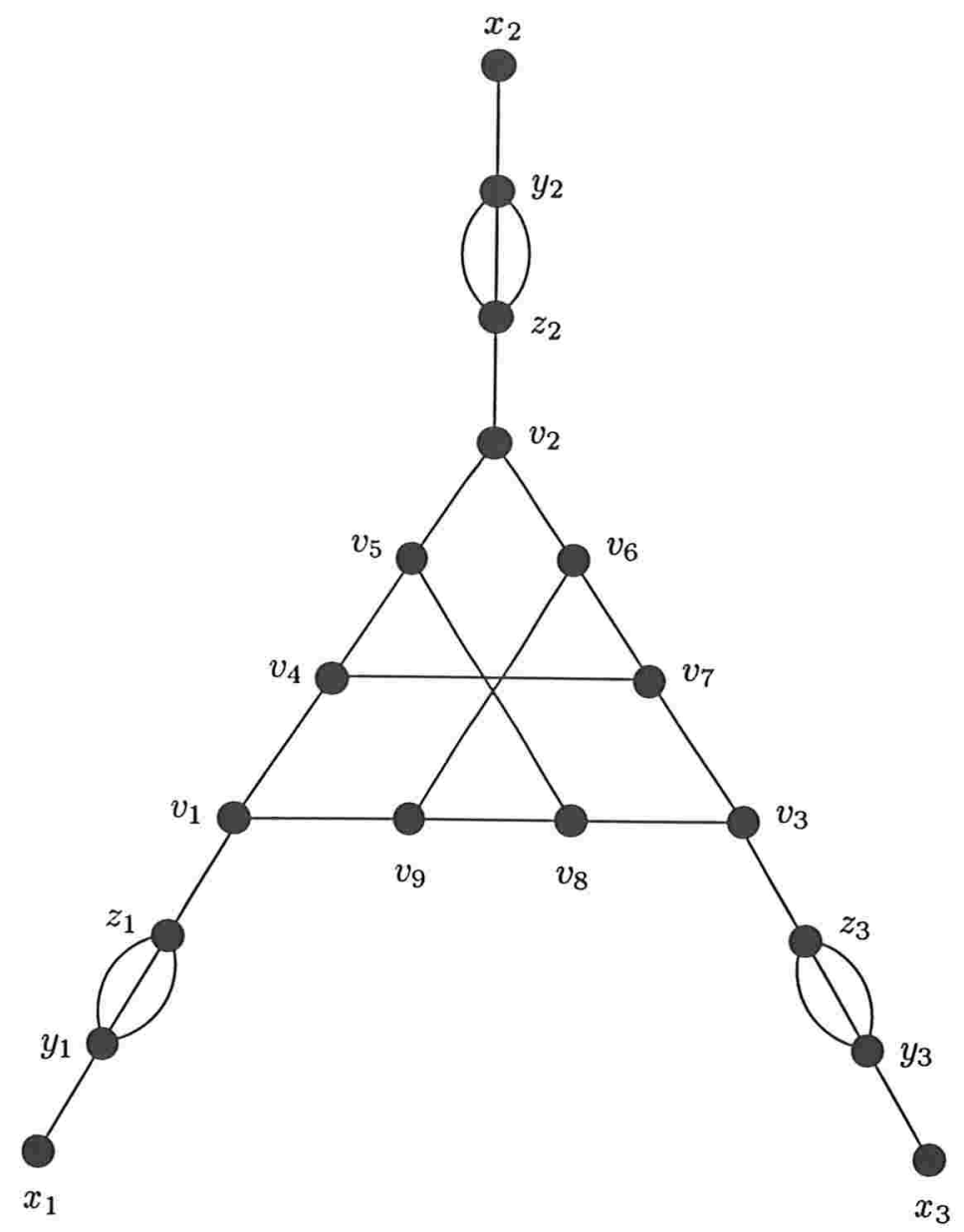

Figura 5.1: O grafo $H$.

aresta ligando $y_{i}$ a $z_{i}$ por um caminho de comprimento $t(i=1,2,3)$, e substituindo cada aresta da forma $v_{i} v_{j}(1 \leq i<j \leq 9)$ por um caminho de comprimento $q$. Vamos chamar cada um dos caminhos de comprimento $t$ (respectivamente, $q$ ) que substituiu uma aresta de $t$-caminho (respectivamente, $q$-caminho). Uma cobertura por caminhos e circuitos de $H(k, q)$ é uma família $\mathcal{F}$ de caminhos e circuitos de $H(k, q)$ que cobre todas as arestas de $H(k, q)$ e cada caminho nesta família tem extremos em $\left\{x_{1}, x_{2}, x_{3}\right\}$. Denotamos por $\ell(\mathcal{F})$ o peso da cobertura $\mathcal{F}$, definido como $\sum_{C \in \mathcal{F}}|C|$.

Se $s_{i}$ é o número de caminhos de $\mathcal{F}$ com um extremo igual a $x_{i}(i=1,2,3)$, dizemos que a cobertura $\mathcal{F}$ é uma $\left(s_{1}, s_{2}, s_{3}\right)$-cobertura. Por simetria, podemos sempre supor que $1 \leq s_{1} \leq s_{2} \leq s_{3}$. É fácil ver que $s_{1}+s_{2}+s_{3}$ é par.

Lema 5.2.1. Sejam $t \geq 16(q+1)>80$ e $\mathcal{F}$ uma $\left(s_{1}, s_{2}, s_{3}\right)$-cobertura por caminhos $e$ circuitos de $H(t, q)$. Então $\ell(\mathcal{F}) \geq 16 q+10 t+12$. Além disso, temos igualdade somente se $\left(s_{1}, s_{2}, s_{3}\right)=(1,2,3)$. 
Prova. Os caminhos

$$
\begin{gathered}
\left(x_{1}, y_{1}, z_{1}, v_{1}, v_{9}, v_{6}, v_{7}, v_{4}, v_{5}, v_{8}, v_{3}, z_{3}, y_{3}, x_{3}\right) \\
\left(x_{2}, y_{2}, z_{2}, v_{2}, v_{5}, v_{4}, v_{1}, v_{9}, v_{8}, v_{3}, z_{3}, y_{3}, x_{3},\right),\left(x_{2}, y_{2}, z_{2}, v_{2}, v_{6}, v_{7}, v_{3}, z_{3}, y_{3}, x_{3}\right)
\end{gathered}
$$

e os circuitos

$$
\left(y_{1}, z_{1}, y_{1}\right),\left(y_{2}, z_{2}, y_{2}\right)
$$

formam uma $(1,2,3)$-cobertura de $H(1,1)=H$ que corresponde de modo natural a uma $(1,2,3)$-cobertura de $H(t, q)$ com peso $16 q+10 t+12$.

Para uma família $\mathcal{C}$ de circuitos/caminhos de um grafo $G$ e um subgrafo $G^{\prime}$ de $G$ seja $\mathcal{C} \mid G^{\prime}:=\left\{C \cap E\left(G^{\prime}\right) \mid C \in \mathcal{C}\right\}$.

Suponha agora que exista uma $\left(s_{1}, s_{2}, s_{3}\right)$-cobertura $\mathcal{F}^{\prime} \operatorname{com} \ell\left(\mathcal{F}^{\prime}\right) \leq 16 q+10 t+12$.

Seja $W_{i}$ o grafo formado pela união dos três $t$-caminhos ligando $y_{i}$ a $z_{i}(1 \leq i \leq 3)$ e $W=W_{1} \cup W_{2} \cup W_{3}$. Seja $\ell\left(\mathcal{F}^{\prime} \mid W\right):=k t$, onde $k \geq 9$ é um inteiro. Não é difícil ver que $k$ é par. Logo, $k \geq 10$.

Afirmamos que $k=10$, ou seja,

$$
\ell\left(\mathcal{F}^{\prime} \mid W\right)=10 t
$$

Se este não for o caso, então $\ell\left(\mathcal{F}^{\prime}\right)>\ell\left(\mathcal{F}^{\prime} \mid W\right) \geq 11 t$. Mas por hipótese, $t \geq 16(q+1)$, e portanto $11 t>16 q+10 t+12$, o que contradiz a escolha de $\mathcal{F}^{\prime}$. Portanto, $\ell\left(\mathcal{F}^{\prime} \mid W\right)=10 t$, e além disso, existe somente um $t$-caminho em $W$ coberto duas vezes por $\mathcal{F}^{\prime}$. Portanto,

(1) somente um dos $s_{i}(i=1,2,3)$ é par;

(2) $s_{i} \leq 4$ para $i=1,2,3$.

Seja $H^{\prime}$ o subgrafo de $H$ induzido pelos $q$-caminhos e pelos vértices $z_{1}, z_{2}, z_{3}$. Assim, o grafo $H(t, q)$ é formado pela união aresta-disjunta dos subgrafos $W_{1}, W_{2}, W_{3}, H^{\prime}$ e das três arestas $x_{1} y_{1}, x_{2} y_{2}, x_{3} y_{3}$. Logo, por (5.1) temos que

$$
\ell\left(\mathcal{F}^{\prime}\right)=10 t+\ell\left(F^{\prime} \mid H^{\prime}\right)+\left(s_{1}+s_{2}+s_{3}\right) .
$$

Note que identificando os vértices $z_{1}, z_{2}, z_{3}$ de $H^{\prime}$ obtemos uma subdivisão de $P_{10}$, o grafo de Petersen.

Suponha que $s_{3}=4$. Por (1), temos que $s_{1}$ e $s_{2}$ são ímpares. Como o grau de cada $v_{i}$ é três, todo vértice $v_{i}(1 \leq i \leq 9, i \neq 3)$ é extremo de algum $q$-caminho coberto um número par de vezes por $\mathcal{F}^{\prime}$. Portanto, existem pelo menos $4 q$-caminhos de $H^{\prime}$ tais que cada um deles é coberto pelo menos duas vezes por $\mathcal{F}^{\prime}$. Note que $H^{\prime}$ possui $12 q$-caminhos e temos $\ell\left(\mathcal{F}^{\prime} \mid H^{\prime}\right) \geq 16 q+8$. Por (5.2) temos que $\ell\left(\mathcal{F}^{\prime}\right) \geq 16 q+10 t+16$. Mas isto é uma contradição e portanto, $s_{3} \leq 3$. Logo, $\left(s_{1}, s_{2}, s_{3}\right)$ é igual a $(2,3,3)$, a $(1,1,2)$ ou a $(1,2,3)$.

Se $\left(s_{1}, s_{2}, s_{3}\right)=(2,3,3)$, então, por um argumento similar ao descrito acima, pelo menos $4 q$-caminhos de $H^{\prime}$ são cobertos duas vezes por $\mathcal{F}^{\prime}$. Logo, temos que $\ell\left(\mathcal{F}^{\prime} \mid H^{\prime}\right) \geq$ $16 q+8$ e, por $(5.2), \ell\left(F^{\prime}\right) \geq 16 q+10 t+16$, o que é uma contradição. Suponha então 
que $\left(s_{1}, s_{2}, s_{3}\right)=(1,1,2)$. Como $\left(P_{10}, p_{10}\right)$ não admite uma cobertura exata por circuitos (veja a Figura 2.1 no Capítulo 2), pelo menos $5 q$-caminhos em $H^{\prime}$ são cobertos duas vezes por $\mathcal{F}^{\prime}$. Logo, $\ell\left(\mathcal{F}^{\prime} \mid H^{\prime}\right) \geq 17 q+4$. Portanto, por $(5.2), \ell\left(\mathcal{F}^{\prime}\right) \geq 17 q+10 t+8$, o que é uma contradição.

Portanto, $\left(s_{1}, s_{2}, s_{3}\right)=(1,2,3)$, e pelo menos $4 q$-caminhos em $H^{\prime}$ são cobertos duas vezes por $\mathcal{F}^{\prime}$. Assim, $\ell\left(\mathcal{F}^{\prime}\right) \geq 16 q+6$, e por (5.2), segue que $\ell\left(\mathcal{F}^{\prime}\right) \geq 16 q+10 t+12$.

De posse do lema acima, vamos provar que o PCMC-G é NP-completo. Para isso, considere o Problema da 3-Aresta-Coloração, o qual foi demonstrado ser NP-completo por Holyer [41]. Esse problema pode ser definido da seguinte forma.

\section{Problema da 3-Aresta-Coloração}

Entrada: Um grafo cúbico $G$.

Questão: $G$ é 3-aresta-colorível?

\section{Teorema 5.2.1 (Thomassen, 1997). O PCMC-G é NP-completo.}

Prova. Seja $G$ uma instância do Problema da 3-Aresta-Coloração com $n:=|V(G)|$. Seja $G^{\prime}$ o grafo obtido a partir de $G$ subdividindo uma vez cada aresta de $G$. A partir de $G^{\prime}$ construa um novo grafo $G^{\prime \prime}$ substituindo cada vértice $v$ de grau três por uma cópia de $H(100,5)$ de modo que os vértices $x_{1}, x_{2}, x_{3}$ tenham grau dois (identifique-os com os vértices novos das subdivisões). O conjunto dos vértices de grau dois que foram inseridos em $G$ para obter $G^{\prime}$ será denotado por $V_{2}$.

Afirmamos que $G$ é 3-aresta-colorível se, e somente se, $G^{\prime \prime}$ possui uma cobertura por circuitos $\mathcal{F}$ com

$$
\ell(\mathcal{F}) \leq 1092 n
$$

Pelo Lema 5.2.1, cada cobertura por caminhos e circuitos de $H(100,5)$ tem peso pelo menos 1092 , e a igualdade ocorre somente se ela for uma $(1,2,3)$-cobertura. Logo, uma cobertura por circuitos $\mathcal{F}$ de $G^{\prime \prime}$ com peso no máximo $1092 n$ rotula $V_{2}$, o qual, de forma natural induz uma 3 -aresta-coloração própria de $G$ com cores $\{1,2,3\}$.

Por outro lado, uma 3-aresta-coloração de $G$ induz uma rotulação de $V_{2}$ e portanto, uma rotulação de $\left\{x_{1}, x_{2}, x_{3}\right\}$ com rótulos 1,2 e 3 para cada cópia de $H(100,5)$. Seja $s_{i}$ a cor de $x_{i}$. A correspondente $\left(s_{1}, s_{2}, s_{3}\right)$-cobertura para cada $H(100,5)$ com peso 1092 fornece uma cobertura por circuitos de $G^{\prime \prime}$ com peso $1092 n$.

O resultado acima implica imediatamente que o PCMC para grafos mistos arbitrários é NP-difícil. Podemos melhorar um pouco este resultado provando que o seguinte problema de decisão é NP-completo. 


\section{PCMC Planar}

Entrada: Um grafo misto planar e um inteiro $k$.

Questão: existe uma cobertura por circuitos de $M$ com peso no máximo $k$ ?

\section{Teorema 5.2.2. O PCMC Planar é NP-completo.}

Prova. Vimos no Capítulo 3 que o PCEC Planar é NP-completo. Ainda, ele é NPcompleto mesmo para grafos mistos planares $(M, p)$ onde $M=(V, E, A)$ tem grau máximo $4, E$ é um emparelhamento, $A$ é um ciclo orientado, $p(e)=2$ para toda aresta $e \in E$ e $p(a)=1$ para todo arco $a \in A$.

Seja $(M, p)$ um grafo misto planar com as propriedades acima. Considere o grafo misto $M^{\prime}$ obtido a partir de $M$ substituindo cada arco $(x, y)$ de $M$ por um caminho orientado de $x$ a $y$ de comprimento $k:=|E|+1$. Afirmamos que $(M, p)$ admite uma cobertura exata por circuitos se, e somente se, existe uma cobertura por circuitos de $M^{\prime}$ com peso no máximo $2|E|+k|A|$. Se $(M, p)$ admite uma cobertura exata então obviamente $M^{\prime}$ possui uma cobertura por circuitos de peso $2|E|+k|A|$.

Suponha agora que exista uma cobertura por circuitos $\mathcal{C}$ de $M^{\prime}$ com peso no máximo $2|E|+k|A|$. Nenhum dos caminhos orientados de comprimento $k$ em $M^{\prime}$ pode ser coberto por mais de um circuito, senão o peso de $\mathcal{C}$ seria ao menos $2 k+k(|A|-1)+|E|=$ $k+k|A|+|E|>2|E|+k|A|$, o que levaria a uma contradição. Logo, cada arco de $M^{\prime}$ é coberto exatamente uma vez. Isto implica necessariamente que cada aresta de $M^{\prime}$ é coberta exatamente duas vezes, e portanto, $\mathcal{C}$ corresponde de maneira natural a uma cobertura exata por circuitos de $(M, p)$. Logo, o PCMC Planar é NP-completo.

\subsection{Casos particulares do PCMC}

Nesta seção apresentamos alguns casos particulares do PCMC para os quais são conhecidos algoritmos polinomiais ou para os quais há evidências de que eles existam. Mostramos que nesses casos o PCMC está intimamente relacionado com o Problema do Carteiro Chinês.

Seja $M$ um grafo misto fortemente conexo e sem pontes. Definimos um passeio de carteiro em $M$ como um passeio fechado $P=\left(v_{1}, e_{1}, v_{2}, \ldots, v_{k}, e_{k}, v_{k+1}=v_{1}\right)$ que passa por todos os arcos e arestas de $M$. Lembre que definimos o comprimento de $P$ como sendo igual a $k$. Nesta seção veremos como os seguintes problemas estão relacionados.

\section{Problema do Carteiro Chinês (PCC)}

Dado um grafo misto fortemente conexo $M$, encontre um passeio de carteiro em $M$ de comprimento mínimo. 


\section{Problema da Cobertura Mínima por Circuitos (PCMC)}

Dado um grafo misto fortemente conexo sem pontes $M$, encontre uma cobertura por circuitos de $M$ de peso mínimo.

Edmonds [24] mostrou que o PCC pode ser resolvido em tempo polinomial quando $M$ é um grafo não-orientado. Edmonds e Johnson [27] mostraram que o PCC também pode ser resolvido eficientemente no caso em que $M$ é um grafo orientado. No entanto, Papadimitriou [54] mostrou que o PCC é NP-difícil mesmo para grafos mistos planares.

Por outro lado, o PCMC é NP-difícil para grafos não-orientados e para grafos mistos planares, como vimos na Seção 5.2.

Denote por $p c c(M)$ e por $\operatorname{cob}(M)$ o valor das soluções ótimas do PCC e do PCMC, respectivamente. É fácil ver que se $M$ é fortemente conexo e sem pontes então $p c c(M) \leq$ $\operatorname{cob}(M)$ (o PCC pode ser formulado como o problema de criar o menor número de cópias de arcos e arestas de $M$ de modo que o grafo resultante seja euleriano). Mas em geral a igualdade não vale. Considere por exemplo o grafo de Petersen $P_{10}$ (veja também a Figura 2.1 no Capítulo 2); temos $p c c\left(P_{10}\right)=20$ mas não é difícil ver que $\operatorname{cob}\left(P_{10}\right)=21$. Outro exemplo é o grafo misto $M K_{4}$ (Figura 2.2 no Capítulo 2); temos $p c c\left(M K_{4}\right)=8$ e $\operatorname{cob}\left(M K_{4}\right)=9$.

Em vista do que foi exposto acima, é natural procurar por classes de grafos mistos $M$ para os quais o PCC e o PCMC são equivalentes, isto é, $p c c(M)=\operatorname{cob}(M)$. Note que para esses grafos é possível calcular o peso de uma cobertura mínima por circuitos em tempo polinomial, mas não é claro se é possível encontrar uma cobertura mínima em tempo polinomial.

A primeira classe que consideramos é a dos grafos orientados. Para tais grafos, claramente o PCC e o PCMC são polinomialmente equivalentes. Mais precisamente, dada uma solução ótima de um dos problemas é possível obter em tempo polinomial uma solução ótima para o outro. Como o PCC em grafos orientados pode ser resolvido em tempo polinomial [24], concluímos que o PCMC também pode ser resolvido eficientemente.

Descrevemos em seguida algumas classes de grafos não-orientados para os quais o PCC e o PCMC são equivalentes. Antes de fazermos isso mais precisamente, é conveniente relembrar alguns conceitos. Dado um grafo não-orientado $G$ e $p: E(G) \mapsto \mathbb{Z}$, dizemos que $(G, p)$ é euleriano se $p(\delta(X))$ é par para todo corte $\delta(X)$. Ainda, dizemos que $(G, p)$ é admissível se é euleriano e $p(\delta(X)-e) \geq p(e)$ para todo corte $\delta(X), e \in \delta(X)$. Quando $p$ assume apenas valores 1 e 2 , é fácil ver que um par euleriano $(G, p)$ é admissivel se, e somente se, $G$ não contém pontes. Vimos no Capítulo 2 que:

1. todo par admissível $(G, p)$, onde $G$ é livre de Petersen, admite uma cobertura exata por circuitos (Teorema 2.4.4),

2. todo par admissível $(G, p)$, onde $G$ admite um 4-fluxo e $p$ assume valores 1 ou 2, admite uma cobertura por circuitos (Lema 2.4.2). 
Não é difícil ver que o PCC pode ser formulado como o problema de encontrar um vetor $p: E(G) \mapsto\{1,2\}$ tal que $(G, p)$ é euleriano e $p(E(G))=\sum_{e \in E(G)} p(e)$ é mínimo. Por esta última observação é imediato que para as classes de grafos acima o PCC e o PCMC são equivalentes.

Teorema 5.3.1. Se G é um grafo 2-aresta-conexo sem menor de Petersen, então $\operatorname{pcc}(G)=\operatorname{cob}(G)$.

Prova. Seja $p: E(G) \mapsto\{1,2\}$ uma solução ótima do PCC na formulação acima. Como $G$ não contém pontes, temos que $(G, p)$ é admissível. Mas $G$ é livre de Petersen, e portanto $(G, p)$ admite uma cobertura exata por circuitos.

Teorema 5.3.2. Se $G$ é um grafo que admite um 4-fluxo, então $p c c(G)=\operatorname{cob}(G)$.

Prova. Similar à prova anterior.

Esses dois resultados trazem algumas implicações algorítmicas interessantes para o PCMC.

Como o PCC pode ser resolvido em tempo polinomial, o Teorema 5.3.1 diz que é possível calcular $\operatorname{cob}(G)$ em tempo polinomial para grafos livres de Petersen. No entanto, ainda não sabemos como encontrar uma cobertura mínima por circuitos em tempo polinomial, a não ser que saibamos resolver a versão de busca do PCEC em tempo polinomial. No entanto, vimos no Capítulo 2 que não se conhece um algoritmo polinomial para este problema. Assim, o problema de encontrar um algoritmo polinomial para o PCMC em grafos livres de Petersen continua ainda em aberto.

Para grafos planares, entretanto, a versão de busca do PCEC pode ser resolvido em tempo polinomial via o Teorema das Quatro Cores [59] (como foi visto no Capítulo 2), e portanto, o PCMC também. Não temos conhecimento de outro algoritmo polinomial para o PCMC em grafos planares que não utilize o Teorema das Quatro Cores. Outro caso que pode ser resolvido em tempo polinomial é quando $G$ é um grafo cúbico livre de Petersen, via uma demonstração da conjectura de Tutte [71] recentemente anunciada por Robertson, Sanders, Seymour e Thomas (Capítulo 2).

O Teorema 5.3.2, por outro lado, é menos útil do ponto de vista algorítmico uma vez que caracterizar grafos que admitem um 4-fluxo é difícil (o problema de decidir se um grafo cúbico admite um 4-fluxo, ou equivalentemente, se é 3-aresta-colorível, é NPcompleto [41]). Uma classe importante de grafos que admitem 4-fluxos (além dos grafos planares) é a dos grafos 4-aresta-conexos [45]. Para esses grafos é possível encontrar um 4-fluxo em tempo polinomial [44], e portanto, o PCMC pode ser resolvido em tempo polinomial para esses grafos. Um problema em aberto é a complexidade do PCMC em grafos não-orientados 3-aresta-conexos. Note que na prova do Teorema 5.2.1 o grafo construído não é 3-aresta-conexo. 
Podemos obter uma versão para o caso misto do Teorema 5.3.1. Antes, vamos relembrar algumas definições vistas no Capítulo 2. Um par $(M, p)$ é euleriano se $\nabla(X)$ é par e satisfaz $f_{p}(X):=p(\delta(X))-\left|p\left(\delta^{-}(X)\right)-p\left(\delta^{+}(X)\right)\right| \geq 0$ para todo corte $\nabla(X)$. Ainda, $(M, p)$ é admissível se é euleriano e $p(\nabla(X)-e) \geq p(e)$ para todo corte $\nabla(X)$ e todo $e \in \delta(X)$. Analogamente ao caso não-orientado, se $p$ assume apenas valores 1 ou 2 , um par euleriano $(M, p)$ é admissível se, e somente se, $M$ não contém pontes. Vimos no Capítulo 2 que todo par admissível $(M, p)$ tal que $M$ é série-paralelo admite uma cobertura exata por circuitos.

Teorema 5.3.3. Se $M$ é um grafo misto série-paralelo fortemente conexo sem pontes, então $\operatorname{pcc}(M)=\operatorname{cob}(M)$.

Prova. Seja $M=(V, E, A)$ um grafo misto série-paralelo fortemente conexo sem pontes. Claramente, $p c c(G) \leq \operatorname{cob}(G)$. Vamos mostrar como obter uma cobertura mínima por circuitos de $M$ a partir de uma solução ótima do PCC.

Seja $P$ um passeio de carteiro de comprimento mínimo em $M$. O passeio $P$ define de modo natural um vetor $p: E \cup A \mapsto \mathbb{Z}_{+}$tal que $(M, p)$ é euleriano: defina $p(e)$ como o número de vezes que $P$ passa por $e$. Vamos mostrar que $(M, p)$ é admissível.

Precisamos do seguinte resultado (fácil de provar): se em um passeio de carteiro de comprimento mínimo uma aresta $e$ é percorrida nas duas direções então ela é percorrida exatamente uma vez em cada direção. Isto significa que se $p(e) \geq 3$ para alguma aresta $e=x y$ então ela é percorrida em apenas uma direção, digamos de $x$ a $y$. Logo, o par $\left(M^{\prime}, p^{\prime}\right)$ obtido de $(M, p)$ orientando $e$ de $x$ a $y$ é euleriano. Portanto, podemos supor que $p(e) \in\{1,2\}$ para toda aresta $e$. Como $M$ não contém pontes, e $p$ é euleriano, temos que $p$ é admissível. Pelo Teorema 2.5.2, concluímos que $(M, p)$ admite uma cobertura exata por circuitos.

Ao contrário do caso não-orientado, o PCC é NP-completo para grafos mistos [54]. No entanto, veremos na Seção 5.4 que tanto o PCC quanto o PCMC podem ser resolvidos em tempo polinomial em grafos série-paralelos, e de modo mais geral, em grafos com largura arbórea limitada.

\subsection{Decomposição e largura arbórea}

O estudo de decomposições arbóreas é relativamente recente (início da década de 80) e trouxe profundas conseqüências a duas áreas: a da teoria dos grafos e a de desenvolvimento de algoritmos. No primeiro caso, decomposições arbóreas e suas propriedades são fundamentais na demonstração do resultado de Robertson e Seymour $[57,56]$ sobre menores em grafos: em toda seqüência infinita de grafos existem dois grafos tais que um deles é menor do outro. No segundo, sabe-se que vários problemas NP-difíceis podem ser resolvidos em tempo polinomial em grafos com largura arbórea limitada por uma constante. Neste capítulo, estamos mais interessados neste último aspecto, em particular, no 
desenvolvimento de algoritmos polinomiais para o PCC e o PCMC em grafos mistos que possuem largura arbórea limitada.

Vejamos inicialmente algumas definições básicas e algumas propriedades fundamentais de decomposições arbóreas.

Seja $G$ um grafo não-orientado. Uma decomposição arbórea de $G$ é um par $(T, \mathcal{W})$, onde $T$ é uma árvore e $\mathcal{W}=\left\{W_{t}: t \in V(T)\right\}$ é uma família de subconjuntos de $V(G)$, tal que:

(T1) $\bigcup_{t \in V(T)} W_{t}=V(G)$;

(T2) para cada aresta $e$ de $G$ existe $t \in V(T)$ tal que ambos os extremos de $e$ pertencem a $W_{t}$;

(T3) se $t, t^{\prime}, t^{\prime \prime} \in V(T)$ e $t^{\prime}$ está no caminho de $t$ a $t^{\prime \prime}$ em $T$, então $W_{t} \cap W_{t^{\prime \prime}} \subseteq W_{t^{\prime}}$.

Note que a condição (T3) acima é equivalente à seguinte:

(T3)' para todo vértice $v$ de $G$ o conjunto $T(v):=\left\{t \in V(T): v \in W_{t}\right\}$ induz uma subárvore de $T$.

As condições (T1) e (T2) juntas dizem que o grafo $G$ é a união dos subgrafos $G\left[W_{t}\right]$. Chamamos cada $W_{t}$ de bolsa. A condição (T3) diz, a grosso modo, que as bolsas de $(T, \mathcal{W})$ estão organizadas em uma estrutura de árvore. Na Figura 5.2 temos um exemplo de uma decomposição arbórea de um grafo $G$. Note que um grafo pode ter várias decomposições arbóreas.

Observação: Para tornar nossa descrição dos algoritmos mais clara vamos nos referir aos elementos de $V(T)$ por nós (em vez de vértices) para distingüi-los dos vértices de $G$ $(M)$. Além disso, escrevemos $t \in T$ em vez de $t \in V(T)$.

A seguinte propriedade separadora caracteriza árvores: a remoção de qualquer aresta (ou vértice interno) separa a árvore em duas subárvores. Um dos aspectos mais importantes de uma decomposição arbórea $(T, \mathcal{W})$ de um grafo $G$ é que ela transfere as propriedades separadoras de $T$ para o grafo $G$. Sejam $X, Y \subseteq V(G)$. Dizemos que $X, Y$ é uma separação de $G$ e que $X \cap Y$ separa $X$ de $Y$ em $G$ se não existem arestas de $G$ com um extremo em $X \backslash Y$ e outro em $Y \backslash X$.

Os três resultados a seguir são a respeito de um grafo não-orientado $G$ com uma decomposição arbórea $(T, \mathcal{W})$.

Lema 5.4.1. Seja $t_{1} t_{2}$ uma aresta qualquer de $T$, e sejam $T_{1}, T_{2}$ os componentes de $T-t_{1} t_{2}$, com $t_{1} \in T_{1}$ e $t_{2} \in T_{2}$. Tome $U_{1}:=\bigcup_{t \in T_{1}} W_{t}$ e $U_{2}:=\bigcup_{t \in T_{2}} W_{t}$. Então $W_{t_{1}} \cap W_{t_{2}}=U_{1} \cap U_{2}$, e $W_{t_{1}} \cap W_{t_{2}}$ separa $U_{1}$ de $U_{2}$ em $G$.

Prova. Para quaisquer $t \in T_{1}$ e $t^{\prime} \in T_{2}, t_{1}$ e $t_{2}$ estão no caminho de $t$ a $t^{\prime}$ em $T$. Assim, por (T3) temos que $U_{1} \cap U_{2} \subseteq W_{t_{1}} \cap W_{t_{2}}$ e portanto $U_{1} \cap U_{2}=W_{t_{1}} \cap W_{t_{2}}$. Resta mostrar 

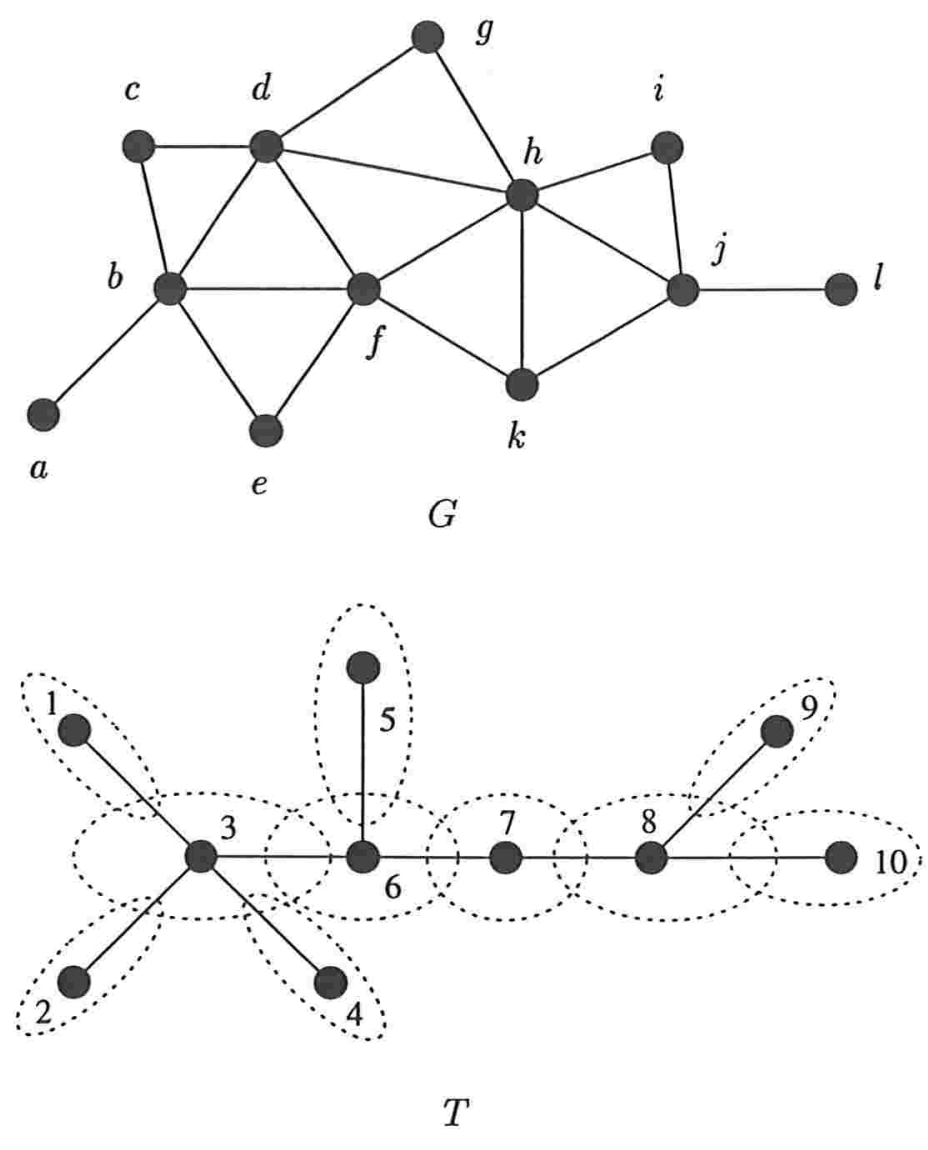

$$
\begin{aligned}
& W_{1}=\{b, c, d\} \\
& W_{2}=\{a, b\} \\
& W_{3}=\{b, d, f\} \\
& W_{4}=\{b, e, f\} \\
& W_{5}=\{d, g, h\} \\
& W_{6}=\{d, f, h\} \\
& W_{7}=\{f, h, k\} \\
& W_{8}=\{h, k, j\} \\
& W_{9}=\{h, i, j\} \\
& W_{10}=\{j, l\}
\end{aligned}
$$

Figura 5.2: Uma decomposição arbórea $\left(T,\left\{W_{1}, \ldots, W_{10}\right\}\right)$ do grafo $G$.

que não existe aresta $u_{1} u_{2}$ com $u_{1} \in U_{1} \backslash U_{2}$ e $u_{2} \in U_{2} \backslash U_{1}$. Se existir uma tal aresta $u_{1} u_{2}$, então por (T2) existe $t \in T$ com $u_{1}, u_{2} \in W_{t}$. Pela escolha de $u_{1}$ e $u_{2}$ temos que $t \notin T_{1}$ e $t \notin T_{2}$, o que é uma contradição.

Lema 5.4.2. Dado $W \subseteq V(G)$, ou existe $t \in T$ tal que $W \subseteq W_{t}$, ou existem vértices $w_{1}, w_{2} \in W$ e uma aresta $t_{1} t_{2}$ tais que $w_{1}, w_{2}$ não pertencem a $W_{t_{1}} \cap W_{t_{2}}$, e são separados por ele em $G$.

Prova. Oriente as arestas de $T$ como segue. Dada uma aresta $t_{1} t_{2} \in T$, defina $U_{1}, U_{2}$ como no Lema 5.4.1; então $W_{t_{1}} \cap W_{t_{2}}=U_{1} \cap U_{2}$ separa $U_{1}$ de $U_{2}$. Se $W_{t_{1}} \cap W_{t_{2}}$ não separa nenhum par de vértices de $W$, então existe $i \in\{1,2\}$ tal que $W \subseteq U_{i}$. Neste caso, oriente $t_{1} t_{2}$ de modo que resulte em um arco entrando em $t_{i}$. Seja $t$ o último vértice de um caminho maximal orientado em $T$; então todas as arestas incidentes a $t$ entram em $t$. Portanto, $W$ está contido na interseção de todos os correspondentes $U_{i}$, e este conjunto é precisamente $W_{t}$. 
Corolário 5.4.1. Todo subgrafo completo de $G$ está contido em alguma bolsa de $(T, \mathcal{W})$.

Abstraindo-se o conteúdo das bolsas de $(T, \mathcal{W})$, o grafo $G$ pode ser visto 'macroscopicamente' como tendo a estrutura da árvore $T$. Entretanto, esta visão macroscópica de $G$ só será relevante se a estrutura de $G$ dentro de cada bolsa for relativamente simples: quanto menor o tamanho das bolsas, maior será a semelhança de $G$ com a árvore $T$.

Esta observação motiva a seguinte definição. A largura de uma decomposição arbórea $(T, \mathcal{W})$ é definida como $\max \left\{\left|W_{t}\right|-1: t \in T\right\}$. A largura arbórea de $G$ é a largura de uma decomposição arbórea de $G$ com menor largura possível. O leitor pode verificar facilmente que árvores são exatamente os grafos simples e conexos com largura arbórea igual a um ${ }^{1}$. Pode-se mostrar que grafos série-paralelos são exatamente os grafos com largura arbórea no máximo dois.

Métodos de programação dinâmica utilizados em árvores podem ser generalizados para grafos com largura arbórea limitada por uma constante fixa $k$. De fato, pode-se dizer que - do ponto de vista algorítmico - o interesse em obter decomposições arbóreas de grafos é fazer uso de técnicas desenvolvidas para árvores na resolução de problemas sobre esses grafos. Muitos problemas bem conhecidos podem ser resolvidos em tempo linear a partir de uma decomposição arbórea de um grafo com largura arbórea limitada: Clique Máximo, Conjunto Independente Máximo, Número Cromático e Circuito/Caminho Hamiltoniano. Mais geralmente, sabe-se que qualquer problema que pode ser formulado como uma fórmula lógica de um certo tipo $[12,21]$ pode ser resolvido em tempo linear em grafos de largura arbórea limitada. Os problemas mencionados pertencem a essa classe. Entretanto, existem outros problemas que podem ser resolvidos em tempo polinomial em grafos com largura arbórea limitada mas não se encaixam nesse paradigma [17, 13]. O PCC e o PCMC são exemplos destes últimos.

Para resolver os problemas acima é necessário que seja dada uma decomposição arbórea de largura no máximo $k$. O problema de encontrar decomposições arbóreas com largura pequena tem sido bem estudado. Robertson e Seymour em um dos artigos da série Graph Minors [58] provaram a existência de um algoritmo polinomial que decide se um grafo possui largura arbórea no máximo $k$ (fixo), mas não o exibiram. Arnborg, Corneil e Proskurowski [11] desenvolveram independentemente um algoritmo $O\left(n^{k+2}\right)$ que determina, para $k$ fixo, se um grafo $G$ com $n$ vértices possui largura arbórea no máximo $k$ e constrói uma decomposição arbórea de $G$ de largura no máximo $k$, se ela existir. Eles provaram também que decidir se um grafo tem largura arbórea no máximo $k$ é NP-completo se $k$ faz parte da entrada. Bodlaender [18] desenvolveu um algoritmo linear que determina, para $k$ fixo, se um grafo possui largura arbórea exatamente $k$ e constrói uma decomposição arbórea de largura $k$, se ela existir. O leitor interessado em mais resultados sobre largura arbórea e menores em grafos pode consultar Diestel [22] e Reed [56].

De modo natural, dado um grafo misto $M$, definimos uma decomposição arbórea para $M$ como uma decomposição arbórea $(T, \mathcal{W})$ do grafo subjacente $G(M)$ de $M$. A largura

\footnotetext{
${ }^{1}$ De fato, o único propósito do '-1' na definição de largura arbórea é tornar esta afirmação verdadeira.
} 
arbórea de $M$ é a largura arbórea de $G(M)$.

Nas próximas seções descreveremos algoritmos polinomiais para o PCC e o PCMC em grafos mistos com largura arbórea limitada. Para tratar desses problemas é mais conveniente pensar que os conjuntos $W_{t}$ induzem uma partição do conjunto dos arcos/arestas de $M$, ou seja, a cada nó $t$ está associado um conjunto de vértices $W_{t}$, um conjunto de $\operatorname{arcos} A\left(W_{t}\right)$ e um conjunto de arestas $E\left(W_{t}\right)$, todos com extremos em $W_{t}$; além disso, cada arco/aresta de $M$ pertence a exatamente um desses conjuntos (se um arco/aresta tem ambos os extremos em mais de uma bolsa, ele é guardado em apenas uma dessas bolsas). No que segue nos referimos a uma bolsa tanto como um conjunto de vértices como um subgrafo de $M$.

Além disso, podemos supor, sem perda de generalidade, que todos os nós de $T$ possuem grau menor ou igual a 3 . De fato, se um nó $t \in T$ possui $d \geq 4$ vizinhos $t_{1}, t_{2}, \ldots, t_{d-1}, t_{d}$ podemos obter uma nova decomposição arbórea $\left(T^{\prime}, \mathcal{W}^{\prime}\right)$ como segue. Seja $t^{\prime}$ um novo nó e remova as arestas $t t_{1}, t t_{2}$ de $T$ e acrescente as novas arestas $t^{\prime} t_{1}, t^{\prime} t_{2}$ e $t^{\prime} t$ obtendo uma nova árvore $T^{\prime}$. Defina $W_{t^{\prime}}:=W_{t}$ e $\mathcal{W}^{\prime}:=\mathcal{W} \cup\left\{W_{t^{\prime}}\right\}$ (esta nova bolsa não contém arcos nem arestas). Claramente, $\left(T^{\prime}, \mathcal{W}^{\prime}\right)$ é uma decomposição arbórea de $G$ com a mesma largura de $(T, \mathcal{W})$. Repetindo este processo um número polinomial de vezes, obtemos uma decomposição arbórea de $G$ com a mesma largura da original onde cada nó da árvore resultante tem grau no máximo 3 . Então $T$ pode ser vista como uma árvore binária com raiz, digamos $r$, onde cada nó $t$ possui no máximo dois filhos. Denote por $T_{t}$ a subárvore de $T$ formada por $t$ e seus descendentes, e por $M_{t}$ o subgrafo de $M$ formado pela união das (arestas e arcos das) bolsas correspondentes aos nós de $T_{t}$.

\subsubsection{O PCC em grafos com largura arbórea limitada}

Nesta seção apresentamos um algoritmo polinomial para o PCC em grafos mistos com largura arbórea limitada. Para facilitar a descrição desse algoritmo, vamos representar uma solução do PCC para um grafo misto $M=(V, E, A)$ de uma forma não usual. Suponha que $P$ seja um passeio de carteiro em $M$. Considere o seguinte supergrafo $H$ de $M$ :

$-V(H)=V$,

- se $e$ aparece $t$ vezes em $P$ então em $H$ existem $t$ cópias de $e$.

Claramente $H$ é um grafo misto euleriano. Portanto, $H$ pode ser escrito como uma união de circuitos disjuntos nos arcos e arestas

$$
E(H) \cup A(H)=C_{1} \cup C_{2} \cup \ldots \cup C_{q} .
$$

Note que alguns desses circuitos podem não corresponder a circuitos de $M$. Isto ocorre exatamente quando um circuito $C_{i}$ é formado de duas cópias de uma mesma aresta de $M$. Dizemos que $C_{i}$ é um pseudocircuito de $M$. Por outro lado, se $C_{1}, \ldots, C_{q}$ é uma coleção de circuitos ou pseudocircuitos de $M$ que cobrem todos os arcos e arestas de $M$ então o grafo $H:=\bigcup_{i} C_{i}$ é euleriano e corresponde a um passeio de carteiro em $M$. 
Definindo o peso de uma coleção $C_{1}, \ldots, C_{q}$ de circuitos e pseudocircuitos como $\sum_{i}\left|C_{i}\right|$, podemos formular o PCC como o problema de encontrar uma coleção de circuitos ou pseudocircuitos de $M$, cobrindo todos os arcos e arestas de $M$, com peso mínimo. Daqui em diante vamos considerar o PCC sob esta formulação, e que $M=(V, E, A)$ é o grafo misto dado.

Uma observação importante é que se $C_{1}, \ldots, C_{q}$ é uma solução ótima do PCC então $q \leq m$, onde $m=|E \cup A|$. Mais especificamente, nenhum arco ou aresta de $M$ aparece em mais de $m$ circuitos ou pseudocircuitos (para $m \geq 2$ ). De fato, suponha que $C_{1}, \ldots, C_{q}$ seja uma solução ótima do PCC. Então para cada (pseudo)circuito $C_{i}$ existe um arco/aresta $e_{i}$ em $C_{i}$ que não aparece em nenhum outro (pseudo)circuito $C_{j}$; caso contrário, poderíamos remover $C_{i}$ da coleção e obter uma solução de menor peso, contrariando a otimalidade.

Agora seguem algumas definições que serão usadas na descrição formal do algoritmo.

Seja $S$ um subconjunto de $V$. Denote por $S^{2}$ o conjunto $\{(u, v): u, v \in S, u \neq v\}$. Uma $S$-configuração é uma função demanda $d: S^{2} \mapsto\{0, \ldots, m\}$.

Seja $d$ uma $S$-configuração, onde $S$ é um subconjunto de vértices de um subgrafo $M^{\prime}$ de $M$. Um par $(\mathcal{P}, \mathcal{C})$, onde $\mathcal{P}$ é uma coleção de caminhos em $M^{\prime}$ com extremos em $S$ e $\mathcal{C}$ é uma coleção de (pseudo)circuitos em $M^{\prime}$, é chamado de realização de $d$ ou uma $d$-realização em $M^{\prime}$ se:

- existe uma partição de $\mathcal{P}=\bigcup_{(u, v) \in S^{2}} Z(u, v)$ tal que $Z(u, v)$ consiste de exatamente $d(u, v)$ caminhos de $u$ a $v$ em $M^{\prime}$ (considere $Z(u, v)=\emptyset$ se $\left.d(u, v)=0\right)$;

- cada arco/aresta de $M^{\prime}$ pertence a algum caminho de $\mathcal{P}$ ou a algum (pseudo)circuito $\operatorname{de} \mathcal{C}$.

Nesse caso, dizemos que a configuração $d$ é realizável em $M^{\prime}$. Definimos o peso de uma $d$-realização $(\mathcal{P}, \mathcal{C})$ como $\sum_{P \in \mathcal{P}}|P|+\sum_{C \in \mathcal{C}}|C|$.

Seja $(T, \mathcal{W})$ uma decomposição arbórea de $M$, onde $T$ é binária com raiz $r$. Observe que, se $t$ é um nó qualquer de $T$ então a restrição de uma solução ótima $\mathcal{C}^{*}$ do PCC ao subgrafo $M_{t}$ corresponde a uma coleção $\mathcal{P}$ de caminhos junto com uma coleção $\mathcal{C}$ de (pseudo)circuitos de $M_{t}$. Não é difícil ver que $(\mathcal{P}, \mathcal{C})$ corresponde a uma $d$-realização de peso mínimo em $M_{t}$ para alguma $W_{t}$-configuração $d$. Outro fato importante é o seguinte: se $\left(\mathcal{P}^{\prime}, \mathcal{C}^{\prime}\right)$ for uma outra $d$-realização de peso mínimo em $M_{t}$, então combinando $\left(\mathcal{P}^{\prime}, \mathcal{C}^{\prime}\right)$ com a restrição de $\mathcal{C}^{*}$ ao subgrafo $M-\left(E\left(M_{t}\right) \cup A\left(M_{t}\right)\right)$ obtemos também uma solução ótima do PCC em $M$.

A estratégia do algoritmo para o PCC que descrevemos a seguir consiste basicamente em encontrar, para cada nó $t$ de $T$ e cada $W_{t}$-configuração $d$, uma $d$-realização de peso mínimo em $M_{t}$. O algoritmo resolve isso inicialmente para as folhas de $T$ e vai sucessivamente considerando os pais dos nós tratados, até chegar à raiz $r$. Note que no caso particular em que $t=r$ e $d \equiv 0$ uma $d$-realização de $M_{t}(=M)$ é uma coleção de pseudocircuitos cobrindo todos os arcos e arestas de $M$, ou seja, uma solução viável do 
PCC. Assim, resolver o PCC equivale a encontrar uma realização em $M$ de peso mínimo da $W_{r}$-configuração $d \equiv 0$.

\subsubsection{Descrição de um algoritmo para o PCC}

A partir de agora suporemos que $M$ possui largura arbórea no máximo $k$ (onde $k$ é uma constante fixa) e que $(T, \mathcal{W})$ é uma decomposição arbórea para $M$ de largura no máximo $k$, onde $T$ é uma árvore binária de raiz $r$. Além disso, podemos supor que cada bolsa $W_{t}$ é simples, e portanto possui no máximo uma certa quantidade fixa (dependente de $k$ ) de arcos e arestas (lembre-se que $M$ pode conter arcos e/ou arestas paralelas). Podemos sempre obter uma decomposição arbórea com essa propriedade simplesmente acrescentando nós e arestas à árvore $T$.

Vejamos agora como usar a decomposição arbórea $(T, \mathcal{W})$ para obter um algoritmo polinomial que encontra uma solução ótima do PCC. Usando a terminologia acima, o algoritmo que desenvolvemos devolve uma realização de peso mínimo para a $W_{r}$-configuração $d \equiv 0$.

O algoritmo mantém em certos nós $t$ uma lista $\mathcal{L}$ de soluções parciais consistindo de ternas $\{d, \mathcal{P}(d), \mathcal{C}(d)\}$ onde $d$ é uma $W_{t}$-configuração e $(\mathcal{P}(d), \mathcal{C}(d))$ é uma $d$-realização de peso mínimo em $M_{t}$. No momento vamos supor que todas as possíveis $W_{t}$-configurações aparecem na lista (isto pode ser reduzido um pouco, como veremos adiante).

Inicialmente o algoritmo constrói as listas para as folhas de $T$. Isto pode ser feito em tempo polinomial uma vez que $\left|W_{t}\right| \leq k+1$ e assim o número de possíveis $W_{t^{-}}$ configurações de uma folha $t$ (e de qualquer nó) é $m^{k(k+1)}$. Para cada uma delas é possível achar uma realização de peso mínimo usando enumeração já que $\left|W_{t}\right|$ é limitado por uma constante.

Então subindo na árvore o algoritmo utiliza as listas de dois nós filhos para obter a lista de soluções parciais do nó pai. Este processo é repetido até chegar na raiz $r$.

Descreveremos agora como é o passo no qual as listas de nós filhos são utilizadas para obter a lista de soluções parciais do nó pai. Suponha que $t$ seja um nó de $T$ e sejam $t_{1}, t_{2}$ os filhos de $t$ (se houver apenas um único filho o procedimento é similar). Sejam $\mathcal{L}_{1}, \mathcal{L}_{2}$ as listas de soluções parciais de $t_{1}, t_{2}$, respectivamente.

Fixe uma $W_{t}$-configuração $d$. Note que se $(\mathcal{P}, \mathcal{C})$ é uma $d$-realização de peso mínimo em $M_{t}$, então para $i=1,2$ a restrição de $(\mathcal{P}, \mathcal{C})$ ao subgrafo $M_{t_{i}}$ é uma realização de peso mínimo em $M_{t_{i}}$ de alguma $W_{t_{i}}$-configuração $d_{i}^{*}$. Lembramos que $M_{t}$ é o subgrafo de $M$ formado pela união dos subgrafos $W_{j}$ correspondentes ao nó $t$ e aos seus descendentes na árvore $T$.

Agora tome duas soluções parciais $\Pi_{i}=\left\{d_{i}, \mathcal{P}\left(d_{i}\right), \mathcal{C}\left(d_{i}\right)\right\} \in \mathcal{L}_{i}, i=1,2$. Uma $d$-extensão do par $\left(\Pi_{1}, \Pi_{2}\right)$ em relação a $d$ é uma $d$-realização $(\mathcal{P}, \mathcal{C})$ em $M_{t}$ com a seguinte propriedade: a restrição de $(\mathcal{P}, \mathcal{C})$ ao subgrafo $M_{t_{i}}$ corresponde à solução parcial $\mathcal{P}\left(d_{i}\right), \mathcal{C}\left(d_{i}\right)$, para $i=1,2$. Não é difícil ver que, se para cada par $\left(\Pi_{1}, \Pi_{2}\right)$ descrito 
acima soubermos calcular uma $d$-extensão de $\left(\Pi_{1}, \Pi_{2}\right)$ de peso mínimo, então uma $d$ realização de peso mínimo em $M_{t}$ pode ser escolhida dentre todas as $d$-extensões de peso mínimo. Obviamente, nem todos os pares $\left(\Pi_{1}, \Pi_{2}\right)$ admitem uma $d$-extensão.

Fixe $d$ e $\Pi_{i}=\left\{d_{i}, \mathcal{P}\left(d_{i}\right), \mathcal{C}\left(d_{i}\right)\right\} \in \mathcal{L}_{i}, i=1,2$. Cada $d_{i}$ está definido em $W_{t_{i}}^{2}$. Note que para o cálculo de uma $d$-extensão do par $\left(\Pi_{1}, \Pi_{2}\right)$ só necessitamos considerar o caso em que o suporte de cada $d_{i}$ está contido em $W_{t}^{2}$. Suponha então que $d_{i}$ esteja definido em $W_{t}^{2}$ ("acrescente zeros"). Para obtermos uma $d$-extensão de $\left(\Pi_{1}, \Pi_{2}\right)$ de peso mínimo defina o grafo $H$ como segue:

$$
\begin{aligned}
& V(H)=W_{t} \\
& E(H)=E\left(W_{t}\right) \\
& A(H)=A\left(W_{t}\right) \cup\left\{(u, v): d_{1}(u, v)+d_{2}(u, v)>0\right\} .
\end{aligned}
$$

Note que estamos supondo que a união acima é disjunta, isto é, mesmo que $(u, v)$ seja um arco da bolsa $W_{t}$, na construção de $H$ acrescentamos um novo arco $(u, v)$. Chamamos esses arcos novos de arcos artificiais. Note que $|V(H)| \leq k+1$ e $|E(H)|+|A(H)| \leq$ $(k+1) k / 2+2(k+1) k=5 k(k+1) / 2$, ou seja, o tamanho de $H$ é limitado por uma constante (lembre-se que $k$ está fixo).

Observação 1. Antes de prosseguirmos, note o seguinte fato. Suponha que $P$ seja um caminho de $u$ a $v$ em $H$. Seja $A^{\prime}$ o conjunto dos arcos artificiais em $P$ e $\mathcal{P}=\left\{P_{a}\right\}_{a \in A^{\prime}}$ uma coleção de caminhos em $M_{t}$ tal que se $a=(i, j)$ então $P_{a}$ é um caminho de $i$ a $j$. Considere o grafo misto $N$ obtido de $P$ substituindo cada arco $a \in A^{\prime}$ pelo caminho $P_{a}$ (faça cópias disjuntas de arcos/arestas se os caminhos se interceptarem). Então não é difícil ver que $N$ pode ser particionado em exatamente um caminho de $u$ a $v$ em $M_{t}$ e vários circuitos e pseudocircuitos de $M_{t}$.

Seja $\mathbf{P}$ a matriz de incidência de $\{$ arestas, arcos $\}$ vs. caminhos de $H$, ou seja, as colunas de $\mathbf{P}$ consistem dos vetores característicos de todos os caminhos em $H$. Analogamente, seja $\mathbf{C}$ a matriz de incidência de $\{$ arestas,arcos\} vs. (pseudo)circuitos de $H$. Denote por $\mathbf{P}_{e}$ (respectivamente $\mathbf{C}_{e}$ ) a linha de $\mathbf{P}$ (respectivamente $\mathbf{C}$ ) correspondente ao arco/aresta $e$. Note que o número de colunas dessas matrizes é limitado por uma constante da ordem de $2^{|E(H)|+|A(H)|} \leq 2^{\frac{5 k(k+1)}{2}}$. Denote por $\mathcal{P}(H, u, v)$ o conjunto dos caminhos de $u$ a $v$ em $H$.

Considere pares $(x, y)$, onde $x$ é um vetor de naturais indexado pelos caminhos de $H$ e $y$ é um vetor de naturais indexado pelos (pseudo)circuitos de $H$, satisfazendo as seguintes condições:

$$
\begin{array}{rlrl}
\sum_{P \in \mathcal{P}(H, u, v)} x_{P} & =d(u, v) & & \forall(u, v) \in V(H)^{2} ; \\
\mathbf{P}_{e} x+\mathbf{C}_{e} y \geq 1 & & \forall e \in E\left(W_{t}\right) \cup A\left(W_{t}\right) ; \\
\mathbf{P}_{a} x+\mathbf{C}_{a} y & =d_{1}(u, v)+d_{2}(u, v) & & \forall a=(u, v) \in A(H) \backslash A\left(W_{t}\right) .
\end{array}
$$

Vamos mostrar que há uma correspondência entre as soluções inteiras $(x, y)$ e as $d$ extensões de $\left(\Pi_{1}, \Pi_{2}\right)$. Claramente, uma $d$-extensão de $\left(\Pi_{1}, \Pi_{2}\right)$ define uma solução inteira $(x, y)$. Suponha que $(x, y)$ seja um vetor de inteiros satisfazendo as restrições 
acima. Seja $\mathcal{P}$ uma coleção de caminhos de $H$ onde cada caminho $P$ aparece exatamente $x_{P}$ vezes e seja $\mathcal{C}$ uma coleção de (pseudo)circuitos de $H$ onde cada (pseudo)circuito $C$ aparece exatamente $y_{C}$ vezes. Lembre-se que o par $\left(\mathcal{P}\left(d_{i}\right), \mathcal{C}\left(d_{i}\right)\right)$ é uma $d_{i}$-realização em $M_{t_{i}}$, para $i=1,2$. O par $(x, y)$ força cada arco artificial $(u, v)$ a aparecer exatamente $d_{1}(u, v)+d_{2}(u, v)$ vezes em $\mathcal{P} \cup \mathcal{C}$. Substituindo cada tal arco $(u, v)$ em $(\mathcal{P}, \mathcal{C})$ por um dos $d_{1}(u, v)+d_{2}(u, v)$ caminhos de $u$ a $v$ em $\mathcal{P}\left(d_{1}\right) \cup \mathcal{P}\left(d_{2}\right)$ obtemos uma coleção de caminhos e (pseudo)circuitos em $M_{t}$ (veja a Observação 1 .), que chamaremos de $\hat{\mathcal{P}}$ e $\hat{\mathcal{C}}$, respectivamente. é fácil ver que $\left(\hat{\mathcal{P}}, \hat{\mathcal{C}} \cup \mathcal{C}\left(d_{1}\right) \cup \mathcal{C}\left(d_{2}\right)\right)$ é uma $d$-realização em $M_{t}$.

Assim, para encontrar uma $d$-extensão de peso mínimo de $\left(\Pi_{1}, \Pi_{2}\right)$, é suficiente encontrar um par $(x, y)$ satisfazendo o sistema de equações e inequações descrito acima que minimize o peso da correspondente realização. Claramente, podemos supor que os componentes de $x$ e $y$ estão no intervalo $[0, m]$. Assim, um limite superior para o número de soluções viáveis do sistema é $m^{f(k)}$, onde $f(k):=O\left(2^{\frac{5 k(k+1)}{2}}\right)$ é a soma do número de colunas de $\mathbf{P}$ com o número de colunas de $\mathbf{C}$.

Logo, dados $d$ e $\Pi_{i}=\left\{d_{i}, \mathcal{P}\left(d_{i}\right), \mathcal{C}\left(d_{i}\right)\right\}, i=1,2$, é possível encontrar uma $d$-extensão de peso mínimo de $\left(\Pi_{1}, \Pi_{2}\right)$ em tempo $O\left(f(k)+m^{f(k)}\right)$. Ainda, dados $d$ e as listas $\mathcal{L}_{1}, \mathcal{L}_{2}$ de soluções parciais de $t_{1}, t_{2}$, pode-se encontrar uma $d$-realização de peso mínimo em $M_{t}$ em tempo $\left|\mathcal{L}_{1}\right| \cdot\left|\mathcal{L}_{2}\right| . O\left(f(k)+m^{f(k)}\right)$. O comprimento de cada lista $\mathcal{L}_{i}$ é no máximo o número de $W_{t_{i}}$-configurações, que é no máximo $m^{k(k+1)}$. Logo, pode-se encontrar uma $d$ realização de peso mínimo em $M_{t}$ em tempo polinomial. Além disso, é possível construir a lista de soluções parciais de um nó $t$ em tempo polinomial a partir das listas dos filhos, repetindo o processo para todas as possíveis $W_{t}$-configurações $d$.

Podemos concluir da discussão acima que o PCC em grafos mistos com largura arbórea limitada pode ser resolvido em tempo polinomial. Naturalmente, devido à complexidade do algoritmo, isso só é viável na prática para valores pequenos de $k$. Pode-se certamente fazer melhorias locais no algoritmo descrito. Por exemplo, em um dado nó $t_{1}$ não é necessário guardar uma lista de soluções parciais com todas as possíveis $W_{t_{1}}$-configurações: basta apenas considerar aquelas que têm suporte em $\left(W_{t_{1}} \cap W_{t}\right)^{2}$ onde $t$ é o pai de $t_{1}$. Mas isso não reduziria a complexidade do algoritmo. A própria estratégia do algoritmo parece limitar as possibilidades de melhorias substanciais na sua complexidade.

$\mathrm{Na}$ próxima seção estenderemos nosso algoritmo para o Problema da Cobertura Mínima por Circuitos.

\subsubsection{O PCMC em grafos com largura arbórea limitada}

Como no caso do PCC, mostraremos aqui como resolver em tempo polinomial o PCMC em grafos mistos com largura arbórea limitada.

Seja $M=(V, E, A)$ um grafo misto com largura arbórea limitada por uma constante $k$ e $(T, \mathcal{W})$ uma decomposição arbórea para $M$ de largura no máximo $k$. Vamos supor sem perda de generalidade que $T$ é binária com raiz $r$ e cada bolsa $W_{t}$ é simples. 
O algoritmo que descreveremos para o PCMC usa o mesmo paradigma do usado para o PCC: construímos listas de soluções parciais para as folhas de $T$ e subimos na árvore combinando as listas de nós filhos para obter uma lista de soluções parciais para o nó pai, obtidas estendendo-se e combinando soluções parciais conhecidas. Nesta última parte, como no PCC, também usaremos uma matriz que enumera as soluções candidatas. Esse algoritmo é uma extensão do algoritmo para o PCC e é conveniente pensar nele desta forma.

A diferença em relação ao PCC reside no fato de que adotaremos uma definição de solução parcial mais complexa para o PCMC. Isto é claramente necessário pois uma solução parcial do PCC não corresponde necessariamente a uma solução parcial do PCMC devido à possível presença de pseudocircuitos.

Vamos introduzir então uma outra definição de solução parcial que evita o problema acima. A motivação para ela é a seguinte. Suponha que $\mathcal{C}^{\approx}$ seja uma solução ótima do PCMC. A restrição de cada circuito $C$ em $\mathcal{C}^{*}$ a um certo subgrafo $M_{t}$ de $M$ consiste de uma coleção de caminhos disjuntos nos vértices (a menos que $C$ esteja contido em $\left.M_{t}\right)$. é natural então considerar tais coleções como estruturas indivisíveis, e usá-las para construir soluções parciais do PCMC. Nas definições seguintes formalizamos essas idéias.

Dado um conjunto $S$ denotamos por $2^{S}$ o conjunto formado por todos os subconjuntos de $S$. Como antes $S^{2}=\{(u, v): u, v \in S, u \neq v\}$ (onde $S$ é o conjunto de vértices de um subgrafo $[S]$ de $M)$. Uma $S$-configuração é uma função demanda $d: 2^{S^{2}} \mapsto\{0, \ldots, m\}$. Chamaremos cada subconjunto $X \subseteq 2^{S^{2}}$ de conjunto terminal em $S$.

Uma c-família em $M$ é uma coleção de caminhos em $M$ dois a dois disjuntos nos vértices. Dado um conjunto terminal $X=\left\{\left(u_{1}, v_{1}\right), \ldots,\left(u_{t}, v_{t}\right)\right\}$ em $S$, dizemos que uma c-família $F=\left\{P_{1}, \ldots, P_{t}\right\}$ cobre $X$ se $P_{i}$ é um caminho de $u_{i}$ a $v_{i}$, para cada $i=1, \ldots, t$.

Seja $d$ uma $S$-configuração, onde $S$ é um subconjunto de vértices de um subgrafo $M^{\prime}$ de $M$. Um par $\left(\mathcal{F}, \mathcal{C}\right.$ ), onde $\mathcal{F}$ é uma coleção de c-famílias em $M^{\prime}$ (com seus caminhos tendo extremos em $S$ ) e $\mathcal{C}$ é uma coleção de circuitos em $M^{\prime}$, é chamado de realização de $d$ ou uma $d$-realização em $M^{\prime}$ se:

- existe uma partição de $\mathcal{F}=\bigcup_{X \in 2^{S^{2}}} Z(X)$ tal que $Z(X)$ consiste de exatamente $d(X)$ c-famílias em $M^{\prime}$ que cobrem $X$;

- cada arco/aresta de $M^{\prime}$ pertence a alguma c-família de $\mathcal{F}$ ou a algum circuito de $\mathcal{C}$.

Nesse caso, dizemos que a configuração $d$ é realizável em $M^{\prime}$. Definimos o peso de uma $d$-realização $(\mathcal{F}, \mathcal{C})$ como $\sum_{F \in \mathcal{F}} \sum_{P \in F}|P|+\sum_{C \in \mathcal{C}}|C|$.

Note que a restrição de uma c-família a um subgrafo $M_{t}$ também é uma c-família (possivelmente cobrindo outro conjunto de vértices) e a restrição de um circuito a $M_{t}$ ou é um circuito ou é uma c-família. Ainda, se $t$ é um nó qualquer de $T$ então a restrição de uma solução ótima $\mathcal{C}^{*}$ do PCMC ao subgrafo $M_{t}$ corresponde a uma coleção $\mathcal{F}$ de c-famílias junto com uma coleção $\mathcal{C}$ de circuitos de $M_{t}$. Não é difícil ver que $(\mathcal{F}, \mathcal{C})$ 
corresponde a uma $d$-realização de peso mínimo em $M_{t}$ para alguma $W_{t}$-configuração $d$. Outro fato importante que devemos mencionar é o seguinte: se $\left(\mathcal{F}^{\prime}, \mathcal{C}^{\prime}\right)$ for uma outra $d$-realização de peso mínimo em $M_{t}$, então combinando $\left(\mathcal{F}^{\prime}, \mathcal{C}^{\prime}\right)$ com a restrição de $\mathcal{C}^{*}$ ao subgrafo $M-\left(E\left(M_{t}\right) \cup A\left(M_{t}\right)\right)$ obtemos também uma solução ótima do PCMC em $M$. Note que no caso particular em que $t=r$ e $d \equiv 0$ uma $d$-realização de $M_{t}(=M)$ é uma coleção de circuitos cobrindo todos os arcos e arestas de $M$, ou seja, uma solução viável do PCMC. Assim, resolver o PCMC equivale a encontrar uma realização em $M$ de peso mínimo da $W_{r}$-configuração $d \equiv 0$.

\subsubsection{Descrição de um algoritmo para o PCMC}

O algoritmo que descreveremos agora usa a decomposição arbórea $(T, \mathcal{W})$ para obter em tempo polinomial uma solução ótima do PCMC em $M$. Usando a terminologia definida na seção anterior, o algoritmo devolve uma realização de peso mínimo para a $W_{r}$-configuração $d \equiv 0$.

O algoritmo mantém em certos nós $t$ uma lista $\mathcal{L}$ de soluções parciais consistindo de ternas $\{d, \mathcal{F}(d), \mathcal{C}(d)\}$ onde $d$ é uma $W_{t}$-configuração e $(\mathcal{F}(d), \mathcal{C}(d))$ é uma $d$ realização de peso mínimo em $M_{t}$. Para simplificar vamos supor que todas as possíveis $W_{t}$-configurações aparecem na lista.

Inicialmente o algoritmo constrói as listas para as folhas de $T$. Isto pode ser feito em tempo polinomial uma vez que $\left|W_{t}\right| \leq k+1$ e assim o número de possíveis $W_{t^{-}}$ configurações de uma folha $t$ é $m^{2^{k(k+1)}}$. Para cada uma delas é possível achar uma realização de peso mínimo usando enumeração já que $\left|W_{t}\right|$ é limitado por uma constante.

Então subindo na árvore o algoritmo utiliza as listas dos dois nós filhos para obter a lista de soluções parciais do nó pai. O algoritmo repete esse processo até chegar a raiz $r$.

Descreveremos agora como é o passo no qual as listas de nós filhos são utilizadas para obter a lista de soluções parciais do nó pai. Suponha que $t$ seja um nó de $T$ e sejam $t_{1}, t_{2}$ os filhos de $t$ (se houver apenas um único filho o procedimento é similar). Sejam $\mathcal{L}_{1}, \mathcal{L}_{2}$ as listas de soluções parciais de $t_{1}, t_{2}$, respectivamente.

Fixe uma $W_{t}$-configuração $d$. Note que se $(\mathcal{F}, \mathcal{C})$ é uma $d$-realização de peso mínimo em $M_{t}$ então para $i=1,2$, a restrição de $(\mathcal{F}, \mathcal{C})$ ao subgrafo $M_{t_{i}}$ é uma realização de peso mínimo em $M_{t_{i}}$ de alguma $W_{t_{i}}$-configuração $d_{i}^{*}$.

Agora tome duas soluções parciais $\Pi_{i}=\left\{d_{i}, \mathcal{F}\left(d_{i}\right), \mathcal{C}\left(d_{i}\right)\right\} \in \mathcal{L}_{i}, i=1,2$. Uma $d$ extensão do par $\left(\Pi_{1}, \Pi_{2}\right)$ é uma $d$-realização $(\mathcal{F}, \mathcal{C})$ em $M_{t}$ com a seguinte propriedade: a restrição de $(\mathcal{F}, \mathcal{C})$ ao subgrafo $M_{t_{i}}$ corresponde à solução parcial $\left(\mathcal{F}\left(d_{i}\right), \mathcal{C}\left(d_{i}\right)\right)$ para $i=1,2$. Mais precisamente:

- $\mathcal{C}\left(d_{1}\right) \cup \mathcal{C}\left(d_{2}\right) \subseteq \mathcal{C}$

- o conjunto de arcos/arestas de cada c-família de $\mathcal{F}$ e de cada circuito de $\mathcal{C}-\left(\mathcal{C}\left(d_{1}\right) \cup\right.$ $\left.\mathcal{C}\left(d_{2}\right)\right)$ pode ser particionado na forma $L \cup F_{1} \cup F_{2}$ onde $L \subseteq E\left(W_{t}\right) \cup A\left(W_{t}\right)$ e para 
$i=1,2$, ou $F_{i}=\emptyset$ ou $F_{i} \in \mathcal{F}\left(d_{i}\right)$;

- cada c-família de $\mathcal{F}\left(d_{i}\right), i=1,2$, está contida em exatamente uma c-família de $\mathcal{F}$ ou em exatamente um circuito de $\mathcal{C}-\left(\mathcal{C}\left(d_{1}\right) \cup \mathcal{C}\left(d_{2}\right)\right)$.

Note que não há perda de generalidade em considerar apenas extensões onde cada nova c-família (ou cada novo circuito) contém no máximo uma c-família de $\mathcal{F}\left(d_{1}\right) \cup \mathcal{F}\left(d_{2}\right)$. Assim, não é difícil ver que, se para cada par $\left(\Pi_{1}, \Pi_{2}\right)$ descrito acima soubermos calcular uma $d$-extensão de $\left(\Pi_{1}, \Pi_{2}\right)$ de peso mínimo, então uma $d$-realização de peso mínimo em $M_{t}$ pode ser escolhida dentre todas as extensões de peso mínimo.

Fixe $d$ e $\Pi_{i}=\left\{d_{i}, \mathcal{F}\left(d_{i}\right), \mathcal{C}\left(d_{i}\right)\right\} \in \mathcal{L}_{i}, i=1,2$. Cada $d_{i}$ está definido em $2^{W_{t_{i}}^{2}}$. Note que para o cálculo de uma $d$-extensão do par $\left(\Pi_{1}, \Pi_{2}\right)$ só necessitamos considerar o caso em que o suporte de cada $d_{i}$ está contido em $2^{W_{t}^{2}}$. Suponha então que $d_{i}$ esteja definido em $2^{W_{t}^{2}}$ ("acrescente zeros").

Como no caso do PCC, queremos reduzir o problema de obter uma $d$-extensão de $\left(\Pi_{1}, \Pi_{2}\right)$ de peso mínimo a um problema de enumerar soluções viáveis de um programa linear inteiro de tamanho constante. Defina o hipergrafo $H$ com conjunto de vértices $V(H)=E\left(W_{t}\right) \cup A\left(W_{t}\right) \cup W_{t}^{2}$ e com o seguinte conjunto de hiper-arestas:

$$
E(H):=\left\{\{e\}: e \in E\left(W_{t}\right) \cup A\left(W_{t}\right)\right\} \cup\left\{X: X \in 2^{W_{t}^{2}}, d_{1}(X)+d_{2}(X)>0\right\} .
$$

Ou seja, $E(H)$ consiste dos conjuntos unitários de arestas e arcos de $W_{t}$ e dos conjuntos terminais em $W_{t}$ com demanda não nula para algum dos $d_{i}$. Consideramos possíveis $\{(u, v)\}$, onde $(u, v)$ é um arco da bolsa $W_{t}$, e um conjunto terminal $X=\{(u, v)\}$ como conjuntos distintos. Note que $|V(H)| \leq k(k+1) / 2+k(k+1)+k(k+1)=5 k(k+1) / 2 \mathrm{e}$ $|E(H)| \leq 3 k(k+1) / 2+2^{k(k+1)}$, ou seja, o tamanho de $H$ é limitado por uma constante ( $k$ está fixo).

Vamos descrever agora o conjunto das soluções viáveis do programa linear inteiro que vamos definir para encontrar extensões de peso mínimo. Suponha que $X, Y$ sejam conjuntos terminais em $W_{t}$ tais que $d_{1}(X)>0$ e $d_{2}(Y)>0$. Seja $F_{X}$ uma c-família em $\mathcal{F}\left(d_{1}\right)$ que cobre $X$ e $F_{Y}$ uma c-família em $\mathcal{F}\left(d_{2}\right)$ que cobre $Y$. Suponha que $L$ seja um subconjunto de $E\left(W_{t}\right) \cup A\left(W_{t}\right)$ tal que $\left\{L, F_{X}, F_{Y}\right\}$ induz uma c-família em $M_{t}$ cobrindo algum subconjunto $S$. Note que este fato independe da escolha de $F_{X}$ ou de $F_{Y}$ (pois os caminhos nas c-famílias são disjuntos). Desse modo, podemos dizer que $L \cup\{X\} \cup\{Y\}$ induz uma c-família em $M_{t}$. De maneira análoga podemos dizer que $L \cup\{X\}$ ou $L \cup\{Y\}$ induz uma c-família em $M_{t}$. Vamos chamar tais conjuntos que induzem c-famílias em $M_{t}$ de c-famílias candidatas em $M_{t}$ com relação a $H$. Dizemos ainda que uma cfamília candidata em $M_{t}$ com relação a $H$ cobre um conjunto $S$ se a correspondente (ou uma das) c-família em $M_{t}$ induzida por ela cobre $S$. De forma similar, dizemos que $L \cup\{X\} \cup\{Y\}$ (resp. $L \cup\{X\}$ ) é um circuito candidato em $M_{t}$ com relação a $H$ se ele induz um circuito em $M_{t}$ (note que este fato novamente independe da escolha de $F_{X}$ ou de $F_{Y}$ ). Note que c-famílias (circuitos) candidatas(os) não são c-famílias (circuitos) em $M_{t}$ mas cada uma (um) representa várias(os) c-famílias (circuitos). 
Através deste artifício, evitamos o problema de enumerar todas as possíveis c-famílias de $M_{t}$. Em vez disso, consideraremos apenas c-famílias candidatas que nada mais são do que triplas de subconjuntos de um conjunto de tamanho limitado por uma constante.

Seja $\mathbf{P}$ a matriz com linhas indexadas por $E(H)$ e cujas colunas consistem de todos os vetores de incidência de c-famílias candidatas em $M_{t}$ com relação a $H$. Analogamente, seja $\mathbf{C}$ a matriz de incidência de $E(H)$ vs. circuitos candidatos em $M_{t}$ com relação a $H$. Denote por $\mathbf{P}_{X}$ (respectivamente $\mathbf{C}_{X}$ ) a linha de $\mathbf{P}$ (respectivamente $\mathbf{C}$ ) correspondente à hiper-aresta $X$ de $H$. No caso particular em que $X=\{e\}$ onde $e$ é um arco/aresta de $W_{t}$ denotamos simplesmente $\mathbf{P}_{e}\left(\mathbf{C}_{e}\right)$. Note que o número de colunas dessas matrizes é limitado por $2^{\left|E\left(W_{t}\right) \cup A\left(W_{t}\right)\right|}\left|2^{W_{t}^{2}}\right|^{2} \leq 2^{3 k(k+1) / 2+2^{k(k+1)}+2 k(k+1)}=2^{7 k(k+1) / 2+2^{k(k+1)}}$. Isto decorre do fato de c-famílias candidatas serem da forma $L \cup\{X\} \cup\{Y\}$ onde $L$ é um subconjunto de arcos e arestas de $W_{t}$ e $X, Y$ são conjuntos terminais em $W_{t}$. Denote por $\mathcal{F}(H, X)$ o conjunto das c-famílias candidatas em $M_{t}$ com relação a $H$ que cobrem $X$.

Considere pares $(x, y)$, onde $x$ é um vetor de naturais indexado pelas c-famílias candidatas em $M_{t}$ com relação a $H$ e $y$ é um vetor de naturais indexado pelos circuitos candidatos em $M_{t}$ com relação $H$, satisfazendo as seguintes condições:

$$
\begin{aligned}
\sum_{F \in \mathcal{F}(H, X)} x_{F} & =d(X) & & \forall X \in 2^{W_{t}^{2}} ; \\
\mathbf{P}_{e} x+\mathbf{C}_{e} y & \geq 1 & & \forall e \in E\left(W_{t}\right) \cup A\left(W_{t}\right) ; \\
\mathbf{P}_{X} x+\mathbf{C}_{X} y & =d_{1}(X)+d_{2}(X) & & \forall X \in E(H) \backslash\left\{\{e\}: e \in E\left(W_{t}\right) \cup A\left(W_{t}\right)\right\} .
\end{aligned}
$$

Vamos mostrar que há uma correspondência entre as soluções inteiras $(x, y)$ e as extensões de $\left(\Pi_{1}, \Pi_{2}\right)$. Claramente, uma extensão de $\left(\Pi_{1}, \Pi_{2}\right)$ define uma solução inteira $(x, y)$. Suponha que $(x, y)$ seja um vetor de inteiros satisfazendo as restrições acima. Seja $\mathcal{F}$ uma coleção de c-famílias candidatas em $M_{t}$ com relação a $H$ onde cada c-família candidata $F$ aparece exatamente $x_{F}$ vezes e seja $\mathcal{C}$ uma coleção de circuitos candidatos em $M_{t}$ com relação a $H$ onde cada circuito candidato $C$ aparece exatamente $y_{C}$ vezes. Lembre-se que o par $\left(\mathcal{F}\left(d_{i}\right), \mathcal{C}\left(d_{i}\right)\right)$ é uma $d_{i}$-realização em $M_{t_{i}}$, para $i=1,2$. O par $(x, y)$ força a ocorrência em $\mathcal{F} \cup \mathcal{C}$ de exatamente $d_{1}(X)+d_{2}(X)$ c-famílias candidatas cobrindo o conjunto terminal $X$. Substituindo essas c-famílias candidatas em $\mathcal{F}, \mathcal{C}$ pelas $d_{1}(X)+d_{2}(X)$ c-famílias em $\mathcal{F}\left(d_{1}\right) \cup \mathcal{F}\left(d_{2}\right)$, para cada conjunto terminal $X$, obtemos uma coleção de c-famílias e circuitos em $M_{t}$, que chamamos de $\hat{\mathcal{F}}$ e $\hat{\mathcal{C}}$, respectivamente. é fácil ver que $\left(\hat{\mathcal{F}}, \hat{\mathcal{C}} \cup \mathcal{C}\left(d_{1}\right) \cup \mathcal{C}\left(d_{2}\right)\right)$ é uma $d$-realização em $M_{t}$.

Assim, para encontrar uma extensão de peso mínimo, é suficiente encontrar um par $(x, y)$ satisfazendo o sistema de equações e inequações descrito acima que minimize o peso da correspondente realização. Claramente, podemos supor que os componentes de $x$ e $y$ estão no intervalo $[0, m]$. Assim, um limite superior para o número de soluções viáveis do sistema é $m^{g(k)}$, onde $g(k):=O\left(2^{7 k(k+1) / 2+2^{k(k+1)}}\right)$ é a soma do número de colunas de $\mathbf{P}$ com o número de colunas de $\mathbf{C}$.

Logo, dados $d$ e $\Pi_{i}=\left\{d_{i}, \mathcal{F}\left(d_{i}\right), \mathcal{C}\left(d_{i}\right)\right\}, i=1,2$, é possível encontrar uma $d$-extensão de peso mínimo de $\left(\Pi_{1}, \Pi_{2}\right)$ em tempo $O\left(g(k)+m^{g(k)}\right)$. Ainda, dados $d$ e as listas $\mathcal{L}_{1}, \mathcal{L}_{2}$ de soluções parciais de $t_{1}, t_{2}$, pode-se encontrar uma $d$-realização de peso mínimo em $M_{t}$ em tempo $\left|\mathcal{L}_{1}\right| \cdot\left|\mathcal{L}_{2}\right| . O\left(g(k)+m^{g(k)}\right)$. O comprimento de cada lista $\mathcal{L}_{i}$ é igual ao número 
de $W_{t_{i}}$-configurações, que é no máximo $m^{2^{k(k+1)}}$. Logo, pode-se encontrar uma realização de $d$ de peso mínimo em $M_{t}$ em tempo polinomial. Além disso, é possível construir a lista de soluções parciais de um nó $t$ em tempo polinomial a partir das listas dos filhos, repetindo o processo para todas as possíveis $W_{t}$-configurações $d$.

A discussão acima mostra que é possível resolver em tempo polinomial o PCMC em grafos mistos de largura arbórea limitada.

\subsubsection{Outros problemas em grafos de largura limitada}

Nesta subseção discutiremos brevemente alguns aspectos sobre a complexidade dos outros problemas estudados neste trabalho em grafos mistos com largura arbórea limitada.

Como vimos no Capítulo 2, o PCEC pode ser resolvido em tempo polinomial para grafos mistos série-paralelos. Como mencionamos anteriormente, grafos série-paralelos são aqueles que possuem largura arbórea no máximo 2. No entanto, a polinomialidade desse caso depende da boa caracterização descrita no Teorema 2.5.2. Para um grafo misto $M=(V, E, A)$ com largura arbórea igual a uma constante $k \geq 3$, não é nem mesmo claro que se um par $(M, p)$ admite uma cobertura exata por circuitos, então existe uma tal cobertura com um número polinomial de circuitos. No entanto, se os pesos $p(e)$ são limitados por um polinômio em $m$ então o PCEC para o par $(M, p)$ pode ser resolvido em tempo polinomial usando uma estratégia similar à usada pelo algoritmo que descrevemos para o PCMC.

Para o PCFC em grafos com largura arbórea limitada a situação é melhor. Como vimos no Capítulo 4, o PCFC pode ser resolvido em tempo polinomial para grafos mistos de uma classe $\mathcal{M}$ se soubermos resolver o PCN para grafos dessa classe. Pode-se mostrar que tanto o PCN como o PCM podem ser resolvido em tempo polinomial em grafos mistos com largura arbórea limitada. Assim, podemos resolver em tempo polinomial o PCFC em grafos mistos com largura arbórea limitada.

\subsection{Algoritmos de aproximação para o PCMC}

Nos últimos anos a o método de abordar problemas NP-difíceis através do desenvolvimento de algoritmos de aproximação tem se tornado bem popular. Como é improvável que existam algoritmos polinomiais exatos que resolvam problemas NP-difíceis faz sentido procurar por algoritmos que devolvam uma solução com uma certa garantia em relação a uma solução ótima. Mais precisamente, dado um problema de minimização $P$, dizemos que um algoritmo $\mathcal{A}$ é de $\alpha$-aproximação para $P$ se para cada instância $I$ de $P$ o custo $c(\mathcal{A}(I))$ da solução $\mathcal{A}(I)$ devolvida pelo algoritmo satisfaz $c(\mathcal{A}(I)) \leq \alpha \cdot O P T(I)$ onde $O P T(I)$ é o custo de uma solução ótima de $I$.

Nesta seção mostraremos um resultado que foi demonstrado por Bermond, Jackson e 
Jaeger [15] e independentemente por Alon e Tarsi [7]: todo grafo 2-aresta-conexo possui uma cobertura por circuitos de peso no máximo $5 / 3|E(G)|$. Embora a motivação desses autores tenha sido a busca por resultados estruturais, veremos que a demonstração dos primeiros pode ser vista como um algoritmo de 5/3-aproximação para o PCMC em grafos não-orientados.

Para descrever o resultado acima é conveniente formular o PCMC da seguinte maneira: dado um grafo não-orientado $G$ sem pontes, encontrar uma família de ciclos $C_{1}, \ldots, C_{k}$ que cobre todas as arestas de $G$ com peso $\sum_{i=1}^{k}\left|C_{i}\right|$ mínimo. Lembre que um ciclo de $G$ é um subgrafo de $G$ onde todos os vértices têm grau par.

Precisamos de dois lemas auxiliares. Para prová-los usamos os dois seguintes teoremas cujas provas são omitidas.

Dado um grafo $G$ e duas arestas adjacentes $e=u v$ e $f=w v$ de $G$, denotamos por $G^{e, f}$ o grafo obtido pelo splitting de $e$ e $f$, isto é, removemos $e$ e $f$ e acrescentamos as arestas $u v^{\prime}$ e $w v^{\prime}$, onde $v^{\prime}$ é um novo vértice. O seguinte resultado mostra que certas operações de splitting preservam 2-aresta-conexidade.

Teorema 5.5.1 (Fleishner [31, 32], 1976). Sejam $G$ um grafo 2-aresta-conexo e $v$ $u m$ vértice grau maior ou igual a 4 . Então existem arestas e ef incidentes a $v$ tais que $G^{e, f}$ é 2-aresta-conexo.

Um r-grafo é um grafo $r$-regular tal que para todo $X \subseteq V(G)$ com $|X|$ ímpar e $0<|X|<|V(G)|,|\delta(X)| \geq r$. Por exemplo, um 3-grafo é um grafo cúbico sem pontes. $\mathrm{O}$ seguinte resultado é um corolário do teorema de Edmonds [25] que caracteriza o poliedro dos emparelhamentos perfeitos. Uma demonstração direta e combinatória foi dada por Seymour [66].

Teorema 5.5.2 (Edmonds, 1965). Seja $G$ um r-grafo. Então existem um inteiro nãonegativo $k$ e uma coleção $\mathcal{F}$ de emparelhamentos perfeitos de $G$ tais que cada aresta de $G$ pertence a exatamente $k$ membros de $\mathcal{F}$.

Lema 5.5.1. Seja $G$ um grafo 2-aresta-conexo. Então existe um ciclo $C$ em $G$ tal que

$$
C \cap B \neq \emptyset
$$

para todo 3-corte $B$ de $G$ e

$$
|C| \geq \frac{2}{3}|E(G)|
$$

Prova. Aplicando o Lema 5.5.1 a $G$ repetidas vezes obtemos um grafo 2-aresta-conexo $H$ com grau máximo 3 . Note que um ciclo em $H$ corresponde de modo natural a um ciclo em $G$, e além disso, cada 3-corte de $G$ também é um 3-corte de $H$.

Considere o grafo cúbico $\bar{H}$. Pelo Teorema 5.5.2, existem um inteiro não-negativo $k$ e uma família $\mathcal{P}$ de emparelhamentos perfeitos de $\bar{H}$ tal que cada aresta de $\bar{H}$ pertence a exatamente $r$ membros de $\mathcal{P}$, e para cada emparelhamento perfeito $\bar{P} \in \mathcal{P}$ e cada 3 -corte $B$ de $\bar{H}$ temos

$$
|\bar{P} \cap B|=1 \text {. }
$$


Note que $|\mathcal{P}|=3 k$.

Para cada emparelhamento perfeito $\bar{P}$ de $\mathcal{P}, P$ denota a coleção de caminhos disjuntos em $G$ correspondentes as arestas de $\bar{P}$. Então

$$
\sum_{\bar{P} \in \mathcal{P}}|P|=k|E(H)| \text {. }
$$

Escolhendo um emparelhamento perfeito $\overline{P_{0}} \in \mathcal{P}$ com $\left|P_{0}\right|$ mínimo, temos

$$
\left|P_{0}\right| \leq \frac{k|E(H)|}{3 k}=\frac{|E(H)|}{3}
$$

e portanto, $C=H-P_{0}$ é um ciclo que satisfaz as condições exigidas.

Lema 5.5.2. Se $G$ é um grafo 2-aresta-conexo e contém um ciclo $C_{1}$ que intercepta todos os 3-cortes de $G$, então $G$ contém dois ciclos $C_{2}, C_{3}$ tais que $\left\{C_{1}, C_{2}, C_{3}\right\}$ é uma cobertura por ciclos de $G$.

Prova. Seja $G^{\prime}:=G / C_{1}$ o grafo obtido a partir de $G$ contraindo as arestas do ciclo $C_{1}$. Claramente, $G^{\prime}$ não possui 3 -cortes. Ainda, podemos eliminar os 2 -cortes de $G^{\prime}$ contraindo uma das arestas desses. O grafo resultante $G^{\prime}$ é 4-aresta-conexo.

Pelo Teorema 2.4.7 (ou veja a Seção 5.3), $G^{\prime}$ admite um 4-fluxo. Assim, existem dois ciclos $C_{2}^{\prime}, C_{3}^{\prime}$ de $G^{\prime}$ tais que $E\left(G^{\prime}\right)=\mathcal{C}_{2}^{\prime} \cup C_{3}^{\prime}$. Acrescentando algumas arestas de $C_{1}$ e possivelmente algumas das arestas contraídas pertencentes a eventuais 2-cortes, podemos estendê-los a ciclos $C_{2}, C_{3}$ em $G$. Logo, $\left\{C_{1}, C_{2}, C_{3}\right\}$ é uma cobertura por ciclos de $G$.

Vamos provar agora o resultado principal.

Teorema 5.5.3 (Bermond, Jackson e Jaeger [15], Alon e Tarsi [7]). Todo grafo 2-aresta-conexo $G$ possui uma cobertura por circuitos (ou ciclos) de peso no máximo $5 / 3|E(G)|$.

Prova. Seja $G$ um grafo 2-aresta-conexo. Pelo Lema 5.5.1, existe um ciclo $C_{1}$ em $G$ que intercepta todos os 3-cortes de $G$ e tal que $\left|C_{1}\right| \geq 2 / 3|E(G)|$. Pelo Lema 5.5.2, existe uma cobertura de ciclos $\left\{C_{1}, C_{2}, C_{3}\right\}$ de $G$ contendo $C_{1}$.

Considere o espaço linear $S:=\left(\mathbb{Z}_{2}\right)^{3}$ e associe a cada $\vec{\alpha}:=\left(\alpha_{1}, \alpha_{2}, \alpha_{3}\right)$ um ciclo

$$
C_{\vec{\alpha}}:=\triangle\left(C_{i}: \alpha_{i}=1, i=1,2,3\right) .
$$

Note que, em particular, $C_{(1,0,0)}=C_{1}$.

É fácil ver que a coleção $\left\{C_{\vec{\alpha}} \mid \vec{\alpha} \in S-\{(0,0,0)\}\right\}$ é uma 4-cobertura exata por ciclos de $G$, ou seja, cada aresta pertence a exatamente 4 ciclos dessa coleção. Logo,

$$
\sum_{\vec{\alpha} \in S-\{(0,0,0)\}}\left|C_{\vec{\alpha}}\right|=4|E(G)| .
$$


Seja $S^{\prime}:=S-\{(0,0,0),(1,0,0)\}$. Pela escolha de $C_{1}$ temos que

$$
\sum_{\vec{\alpha} \in S^{\prime}}\left|C_{\vec{\alpha}}\right| \leq 4|E(G)|-\frac{2}{3}|E(G)|=\frac{10}{3}|E(G)| .
$$

Seja $\mathcal{B}$ o conjunto das bases de $S$ que não contêm $(1,0,0)$. Não é difícil ver que se $\{\vec{\alpha}, \vec{\beta}, \vec{\gamma}\}$ é uma base em $\mathcal{B}$ então $\left\{C_{\vec{\alpha}}, C_{\vec{\beta}}, C_{\vec{\gamma}}\right\}$ é uma cobertura por ciclos de $G$. Queremos mostrar que existe uma base $B_{0} \in \mathcal{B}$ tal que $\sum_{\vec{\alpha} \in B_{0}}\left|C_{\vec{\alpha}}\right| \leq 5 / 3|E(G)|$.

Note que cada vetor de $S^{\prime}$ pertence a exatamente 8 bases de $\mathcal{B}$. Logo,

$$
\sum_{B \in \mathcal{B}} \sum_{\vec{\alpha} \in B}\left|C_{\vec{\alpha}}\right|=8 \sum_{\vec{\alpha} \in S^{\prime}}\left|C_{\vec{\alpha}}\right| \leq \frac{80}{3}|E(G)| .
$$

Como $\mathcal{B}$ contém exatamente 16 bases, existe uma base $B_{0}$ tal que

$$
\sum_{\vec{\alpha} \in B_{0}}\left|C_{\vec{\alpha}}\right| \leq \frac{1}{16} \cdot \frac{80}{3}|E(G)|=\frac{5}{3}|E(G)| .
$$

Isto conclui a prova.

Vamos agora mostrar que a demonstração acima pode ser transformada em um algoritmo polinomial de aproximação para o PCMC. Não é difícil ver que uma possível implementação desse algoritmo depende principalmente de sabermos resolver os seguintes subproblemas:

1. encontrar uma família $\mathcal{P}$ de emparelhamentos de um 3 -grafo $\widetilde{H}$ satisfazendo as condições descritas na prova do Lema $5.5 .1 \mathrm{e}$

2. encontrar um 4-fluxo em um grafo 4-aresta-conexo como na prova do Lema 5.5.2.

O primeiro desses subproblemas é equivalente a encontrar vetores de incidência de emparelhamentos tais que $\chi^{E(\widetilde{H})}$ é combinação convexa desses (isso segue da caracterização de Edmonds [25] do poliedro dos emparelhamentos perfeitos) e pode ser feito em tempo polinomial (veja por exemplo [39]). O segundo subproblema também pode ser resolvido em tempo polinomial [44]. Obtemos então um algoritmo de $5 / 3$-aproximação para o PCMC.

Podemos estender esse algoritmo para a versão mais geral do PCMC onde são dados um grafo 2-aresta-conexo $G$ com uma função peso $w: E(G) \mapsto \mathbb{Z}_{+}$e queremos encontrar uma cobertura por circuitos (ou ciclos) $\mathcal{C}$ de $G$ com peso mínimo. Note que é possível proceder de modo similar à prova do Lema 5.5.1 para obter um ciclo em $G$ que intercepta todos os 3-cortes de $G$ e com peso $w(C) \leq 2 / 3 w(E(G))$ (basicamente substituindo |.| por $w($.$) onde for apropriado). A mesma técnica da prova do Teorema 5.5.3, agora levando$ em conta os pesos, pode ser usada para encontrar uma cobertura por ciclos de $G$ com peso no máximo $5 / 3 w(E(G))$. 
Alon e Tarsi [7] conjecturaram que o limite superior do Teorema 5.5.3 poderia ser reduzido.

Conjectura 5.5.1. Todo grafo 2-aresta-conexo $G$ contém uma cobertura por ciclos de peso no máximo $7 / 5|E(G)|$.

Note que isto seria o melhor possível pois para o grafo de Petersen temos

$$
\frac{\operatorname{cob}\left(P_{10}\right)}{\left|E\left(P_{10}\right)\right|}=\frac{21}{15}=\frac{7}{5} \text {. }
$$

A conjectura acima continua em aberto mas Jamshy e Tarsi [46] mostraram o seguinte.

Teorema 5.5.4 (Jamshy e Tarsi, 1992). A Conjectura 5.5.1 implica a conjectura da cobertura dupla por circuitos (Conjectura 2.7.1 do Capítulo 2).

Não incluímos aqui a demonstração desse resultado mas ressaltamos aqui que o grafo de Petersen desempenha um papel importante nessa prova.

Concluindo esta seção mencionamos que não se conhecem algoritmos de $\alpha$ aproximação para o PCMC em grafos mistos arbitrários.

$\mathrm{Na}$ Tabela 5.1 apresentamos um resumo dos principais resultados sobre o PCMC discutidos neste capítulo, com exceção do resultado de aproximação que acabamos de ver. 


\begin{tabular}{|c|c|c|c|}
\hline \multicolumn{4}{|c|}{ PCMC } \\
\hline \multicolumn{2}{|c|}{ Grafo } & Complexidade & Referência \\
\hline \multicolumn{2}{|l|}{ Orientado } & Polinomial & {$[27]$} \\
\hline \multirow{4}{*}{ Não-orientado } & 4-aresta-conexo & Polinomial & [44] \\
\hline & Planar & Polinomial & {$[15,40,59]$} \\
\hline & Livre de Petersen & $\begin{array}{c}\text { Polinomial } \\
\text { (sabe-se apenas calcular } \\
\text { o valor ótimo) }\end{array}$ & {$[2]$} \\
\hline & Arbitrário & NP-difícil & {$[70]$} \\
\hline \multirow{3}{*}{ Misto } & $\begin{array}{l}\text { Largura arbórea } \\
\text { limitada }\end{array}$ & Polinomial & Seção 5.4 .4 \\
\hline & Planar & NP-difícil & Seção 5.2 \\
\hline & Arbitrário & NP-difícil & {$[70]$} \\
\hline
\end{tabular}

Tabela 5.1: Tabela dos principais resultados sobre o PCEC. 


\section{A conjectura de Woodall}

\subsection{Introdução}

Em 1978 Woodall conjecturou que em um grafo orientado planar o comprimento de um circuito mínimo é igual à cardinalidade de um coleção máxima de transversais disjuntas.

Neste capítulo provamos que a igualdade acima é verdadeira para grafos orientados série-paralelos. $\mathrm{Na}$ verdade, provamos uma versão mais geral envolvendo pesos (capacidades), da qual segue o último resultado.

\subsection{Definições e resultados conhecidos}

Seja $D=(V, A)$ um grafo orientado. Lembre que um subconjunto não vazio de arcos $B$ é um dicorte de $D$ se existe $X \subseteq V$ tal que $\delta^{-}(X)=\emptyset$ e $B=\delta^{+}(X)$. Uma junção de $D$ é um subconjunto de arcos que intercepta todo dicorte de $D$. Uma transversal (de circuitos) de $D$ é um subconjunto de arcos que intercepta todo circuito de $D$. Note que para grafos orientados planos existe uma correspondência entre dicortes (junções) e circuitos (transversais) nos grafos orientados duais correspondentes.

Claramente, a cardinalidade de uma coleção com o maior número possível de junções disjuntas é menor ou igual ao tamanho de um dicorte mínimo. Woodall [73] conjecturou o seguinte.

Conjectura 6.2.1 (Woodall, 1978). Em qualquer grafo orientado o tamanho de um dicorte mínimo é igual à cardinalidade de uma coleção máxima de junções disjuntas.

Dada uma função peso $l: A \mapsto \mathbb{Z}_{+}$, denote por $\operatorname{Dic}(D, l)$ o peso de um dicorte de peso mínimo com respeito a $l$. Dada $l$, dizemos que uma coleção $\mathcal{F}$ de subconjuntos de $A$ é l-disjunta se cada $a \in A$ pertence a no máximo $l(a)$ membros de $\mathcal{F}$. Denote por $\operatorname{Jun}(D, l)$ a cardinalidade de uma coleção máxima $l$-disjunta formada por junções. Claramente, $\operatorname{Jun}(D, l) \leq \operatorname{Dic}(D, l)$. 
Edmonds e Giles conjecturaram que para todo grafo orientado $(D, l), \operatorname{Jun}(D, l)=$ $\operatorname{Dic}(D, l)$. Note que isto seria uma generalização da conjectura de Woodall. No entanto, Schrijver [61] mostrou que a conjectura de Edmonds e Giles é falsa ao exibir o seguinte contra-exemplo.

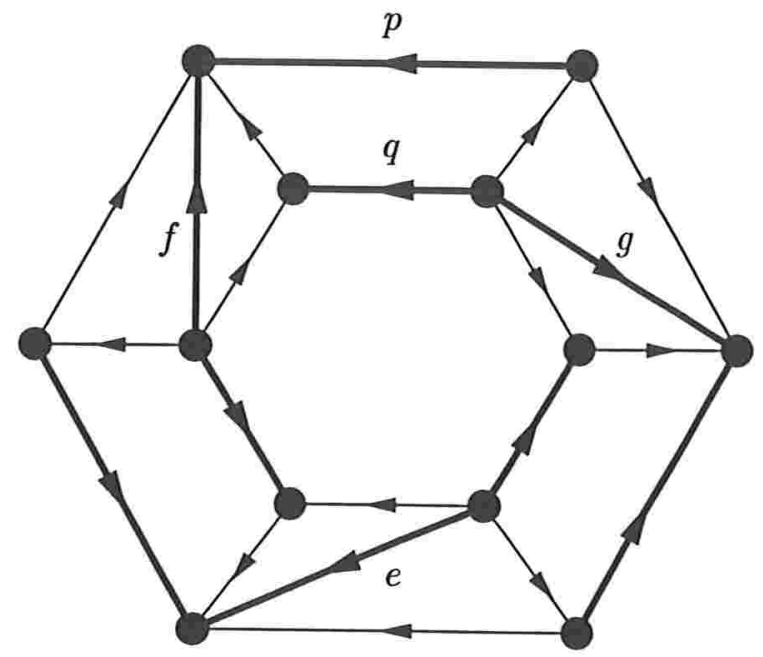

Figura 6.1: Contra-exemplo de Schrijver. Os arcos em negrito têm peso 1 enquanto os demais têm peso 0 .

Para verificar que o grafo $(D, l)$ da Figura 6.1 é de fato um contra-exemplo considere o seguinte. O peso de um dicorte mínimo em $(D, l)$ é 2 . Suponha que existam duas junções $l$-disjuntas $J_{1}, J_{2}$. Note que essas junções só podem conter arcos de peso 1 . Os seis vértices do hexágono interno da Figura 6.1 induzem um dicorte contendo os arcos $e, f$ e $g$. Como cada um destes arcos pertence a um dicorte trivial de peso 2 , e $J_{1}, J_{2}$ são junções $l$-disjuntas, um desses arcos pertence a $J_{1}$ e os outros dois a $J_{2}$, ou vice-versa. Por simetria, podemos supor que $e \in J_{1}$ e $f, g \in J_{2}$. Isso implica que os arcos $p$ e $q$ pertencem a $J_{1}$. Mas então o dicorte contendo $p, q$ e $e$ não contém nenhum arco de $J_{2}$, o que contraria o fato deste ser uma junção. Logo, não existem duas junções $l$-disjuntas em $D$.

A Conjectura 6.2.1 continua ainda em aberto. O melhor resultado conhecido é o de Feofiloff e Younger [33], e Schrijver [62]. Eles provaram independentemente que $\operatorname{Jun}(D, l)=\operatorname{Dic}(D, l)$ para grafos orientados com conexão fonte-sorvedouro (são grafos orientados para os quais existe um caminho de cada fonte a cada sorvedouro).

Se nos restringirmos a grafos orientados planares temos uma conjectura dual à Conjectura 6.2.1.

Conjectura 6.2.2 (Woodall, 1978). Em um grafo orientado planar o comprimento de um circuito mínimo é igual à cardinalidade de uma coleção máxima de transversais disjuntas.

Note que o contra-exemplo da Figura 6.1 é planar e portanto, a versão com pesos da 
conjectura acima não vale, isto é, o peso de um circuito mínimo de um grafo orientado planar $(D, l)$ nem sempre é igual à cardinalidade de uma coleção máxima $l$-disjunta formada de transversais.

Uma pergunta natural é se a hipótese do grafo orientado ser planar na conjectura pode ser removida. A resposta é não, como nos foi contado por D.H. Younger. Segundo ele, o seguinte contra-exemplo foi encontrado por Thomassen. Sejam $X:=\left\{x_{1}, \ldots, x_{5}\right\}, Y:=$ $\left\{y_{1}, \ldots, y_{5}\right\}$ e $Z=\left\{z_{1}, \ldots, z_{5}\right\}$ conjuntos disjuntos. O contra-exemplo é um torneio $\Gamma$ com 15 vértices, onde $V(\Gamma)=X \cup Y \cup Z$. Os grafos orientados induzidos $\Gamma[X], \Gamma[Y], \Gamma[Z]$ são escolhidos de modo que o tamanho de uma transversal mínima de cada um deles seja ao menos 3. Existem 5 triângulos orientados $\left(x_{i}, y_{i}, z_{i}\right), i=1, \ldots, 5$. Os demais arcos vão de $Y$ a $X, X$ a $Z$ e $Z$ a $Y$. A cintura de $\Gamma$ é 3. Pode-se mostrar que o tamanho de uma transversal mínima de $\Gamma$ é maior que $35=105 / 3=|A(\Gamma)| / 3$ e portanto, $\Gamma$ não possui 3 transversais disjuntas.

\subsection{Prova da conjectura para grafos série-paralelos}

Nesta seção provamos que a Conjectura 6.2.2 vale para grafos orientados sérieparalelos. $\mathrm{Na}$ verdade, provamos uma versão mais geral na qual os arcos têm pesos associados. Dados um grafo orientado $D=(V, A)$ e uma função peso $l: A \mapsto \mathbb{Z}_{+}$, denote por $\operatorname{Cint}(D, l)$ o peso de um circuito mínimo de $(D, l)$, e por $\operatorname{Trans}(D, l)$ a cardinalidade de uma coleção máxima $l$-disjunta de transversais de $D$.

Teorema 6.3.1. Sejam $D$ um grafo orientado série-paralelo e $l: A(D) \mapsto \mathbb{Z}_{+}$uma função peso. Então

$$
\operatorname{Cint}(D, l)=\operatorname{Trans}(D, l) .
$$

Ou seja, o peso de um circuito mínimo é igual à cardinalidade de uma coleção l-disjunta máxima de transversais.

Prova. Claramente, Trans $(D, l) \leq \operatorname{Cint}(D, l)$. Assim, basta provar a desigualdade inversa. Faremos isto por indução no tamanho de $D$.

Para facilitar nossa apresentação, nesta prova usaremos a notação simplificada $x y$ para denotar um arco $(x, y)$.

Seja $D=(V, A)$ um grafo orientado série-paralelo. No que segue reduziremos sempre o par $(D, l)$ a um par $\left(D^{\prime}, l^{\prime}\right)$ tal que $\operatorname{Cint}\left(D^{\prime}, l^{\prime}\right)=\operatorname{Cint}(D, l)$.

Se $D$ possui apenas dois vértices o resultado é imediato. Então suponha que $D$ possua ao menos três vértices e que $\operatorname{Cint}(D, l)=k>0$.

Se $a=x y$ e $b=y z$ são dois arcos em série (ou seja, $y$ tem grau 2, e $x \neq y$ ), então definimos um novo grafo orientado $D^{\prime}:=D-y+f$, onde $f=x z$ é um novo arco. Seja $l^{\prime}$ uma função peso definida sobre os arcos de $D^{\prime}$ da seguinte forma:

$$
l^{\prime}(e):= \begin{cases}l(a)+l(b), & \text { se } e=f \\ l(e) & \text { caso contrário. }\end{cases}
$$


Note que $\operatorname{Cint}(D, l)=\operatorname{Cint}\left(D^{\prime}, l^{\prime}\right)$. Além disso, temos que $\operatorname{Trans}\left(D^{\prime}, l^{\prime}\right) \leq \operatorname{Trans}(D, l)$. De fato, dada uma coleção $l^{\prime}$-disjunta $F_{1}^{\prime}, \ldots, F_{r}^{\prime}$ de transversais de $D^{\prime}$, podemos obter a partir desta uma coleção $l$-disjunta $F_{1}, \ldots, F_{r}$ de transversais de $D$ como segue. Para cada $F_{i}^{\prime}$ que contém $f$ substituímos $f$ pelos arcos $a$ e $b$ de modo que $a$ não apareça em mais que $l(a)$ conjuntos e $b$ não apareça em mais que $l(b)$ conjuntos. É fácil ver que cada membro da coleção resultante é uma transversal de $D$ e que essa coleção é $l$-disjunta. Pela hipótese de indução, $\operatorname{Cint}\left(D^{\prime}, l^{\prime}\right)=\operatorname{Trans}\left(D^{\prime}, l^{\prime}\right)$. Portanto,

$$
\operatorname{Cint}(D, l)=\operatorname{Cint}\left(D^{\prime}, l^{\prime}\right)=\operatorname{Trans}\left(D^{\prime}, l^{\prime}\right) \leq \operatorname{Trans}(D, l),
$$

e o resultado segue.

Se $a=x y$ e $b=x y$ são dois arcos paralelos com $l(a) \leq l(b)$, então tome $D^{\prime}:=D-b$ com função peso $l^{\prime}$ definida como $l^{\prime}(e)=l(e)$ para $e \in \bar{A}-b$. Note que $\operatorname{Cint}\left(D^{\prime}, l^{\prime}\right)=$ $\operatorname{Cint}(D, l)$. Além disso, temos Trans $\left(D^{\prime}, l^{\prime}\right) \leq \operatorname{Trans}(D, l)$. De fato, dada uma coleção $l^{\prime}$-disjunta $F_{1}^{\prime}, \ldots, F_{r}^{\prime}$ de transversais de $D^{\prime}$, podemos obter a partir desta uma coleção $l$-disjunta $F_{1}, \ldots, F_{r}$ de transversais de $D$ tomando $F_{i}:=F_{i}^{\prime} \cup\{b\}$ se $F_{i}^{\prime}$ contém $a$, e $F_{i}:=$ $F_{i}^{\prime}$, caso contrário. Usando a hipótese de indução e os fatos previamente mencionados, temos

$$
\operatorname{Cint}(D, l)=\operatorname{Cint}\left(D^{\prime}, l^{\prime}\right)=\operatorname{Trans}\left(D^{\prime}, l^{\prime}\right) \leq \operatorname{Trans}(D, l),
$$

e o resultado segue.

Então suponha que $D$ não contenha nem arcos em série nem arcos paralelos. Além disso, podemos supor que $D$ não possui vértices de corte.

Para facilitar nossa apresentação suporemos que $(D, l)$ é crítico, isto é, todo arco $a$ com peso positivo está contido em algum circuito mínimo. Se isto não é o caso para algum arco $a=x y$, então podemos diminuir $l(a)$ convenientemente definindo $l^{\prime \prime}(a):=$ $\max \{0, k-\alpha\}$, onde $\alpha$ é o peso de um caminho mínimo de $y$ a $x$. Repetindo esta operação para todo arco $a$, obtemos um grafo orientado crítico $\left(D, l^{\prime \prime}\right)$ para algum $l^{\prime \prime} \leq l$. Então é suficiente mostrar que existe uma coleção $l^{\prime \prime}$-disjunta de $k$ transversais de $\bar{D}$, pois ela também será $l$-disjunta.

Assim, suponha que $(D, l)$ seja crítico. Em particular, temos $l(a) \leq k$ para todo arco $a$ de $D$.

É um fato bem conhecido que todo grafo série-paralelo sem vértices de corte possui um vértice com exatamente 2 vizinhos (veja o Lema 2.6 .1 no Capítulo 2). Seja y um vértice em $D$ com exatamente dois vizinhos, digamos $x$ e $z$. Temos duas possibilidades a analisar.

Caso 1. $x y, y z, z y \in A$ e $y x \notin A$. (O caso $y x, y z, z y \in A$ e $x y \notin A$ pode ser tratado de modo similar.)

Considere o grafo orientado $D^{\prime}:=D-z y$ e tome $l^{\prime}(e):=l(e)$ para $e \in A-z y$ (veja a Figura 6.2). Como $D$ é crítico, e $k>0$, segue que $\operatorname{Cint}\left(D^{\prime}, l^{\prime}\right)=\operatorname{Cint}(D, l)=$ $l(y z)+l(z y)=k$, a menos que $D^{\prime}$ não possua circuitos, caso no qual é imediato que $\operatorname{Cint}(D, l)=\operatorname{Trans}(D, l)$. 




$D$

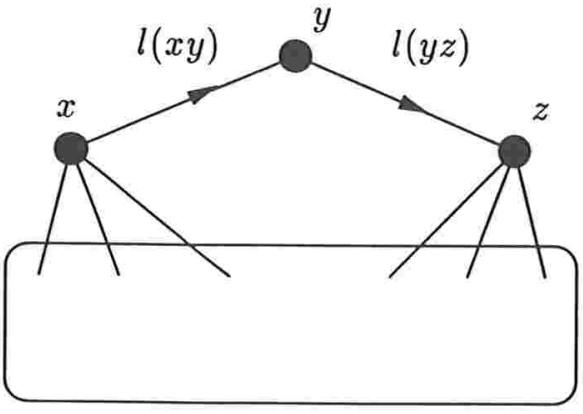

$D^{\prime}$

Figura 6.2: Caso 1 da prova do Teorema 6.3.1.

Pela hipótese de indução, existe uma coleção $l^{\prime}$-disjunta $F_{1}^{\prime}, \ldots, F_{k}^{\prime}$ de $k$ transversais de $D^{\prime}$. Podemos supor que $y z$ pertence a exatamente $l(y z)$ dessas transversais. Podemos obter a partir dessa coleção de transversais de $D^{\prime}$ uma coleção $l$-disjunta $F_{1}, \ldots, F_{k}$ de transversais de $D$ da seguinte forma. Defina

$$
F_{i}:= \begin{cases}F_{i}^{\prime}, & \text { se } F_{i}^{\prime} \text { contém yz } \\ F_{i}^{\prime}+z y, & \text { caso contrário. }\end{cases}
$$

Logo, $\operatorname{Trans}(D, l) \geq k=\operatorname{Cint}(D, l)$, e o resultado segue.

Caso 2. $x y, y x, y z, z y \in A$.

Considere os circuitos $C_{1}=\{x y, y x\}$ e $C_{2}=\{z y, y z\}$. Note que qualquer circuito distinto de $C_{1}$ que contém o arco $x y$ deve conter o arco $y z$; do mesmo modo, qualquer circuito distinto de $C_{2}$ que contém o arco $y z$ deve conter o arco $x y$. Argumentos similares se aplicam aos arcos $y x$ e $z y$.

Considere o grafo orientado $D^{\prime}:=D-y \cup\{x z, z x\}$, e tome $l^{\prime}(e)=l(e)$ para $e \in$ $A-\{x y, y x, y z, z y\}, l^{\prime}(x z):=l(x y)+l(y z)$ e $l^{\prime}(z x):=l(z y)+l(y x)$ (veja a Figura 6.3). É imediato que $\operatorname{Cint}(D, l) \leq \operatorname{Cint}\left(D^{\prime}, l^{\prime}\right)$. Se $\operatorname{Cint}(D, l)<\operatorname{Cint}\left(D^{\prime}, l^{\prime}\right)$, então isto significa que todo arco distinto de $x y, y x, y z, z y$ tem peso zero (lembre-se que $(D, l)$ é crítico). Ainda, isto implica que todo circuito em $D$ contém um desses arcos (caso contrário $k=\operatorname{Cint}(D, l)=0)$. Suponha agora que $\operatorname{Cint}(D, l)=\operatorname{Cint}\left(D^{\prime}, l^{\prime}\right)$. Pela hipótese de indução, existe uma coleção $l^{\prime}$-disjunta $F_{1}^{\prime}, \ldots, F_{k}^{\prime}$ de transversais de $D^{\prime}$.

Note que para cada $i, F_{i}^{\prime \prime}:=F_{i}^{\prime}-\{x z, z x\}$ é quase uma transversal de $D$ : ela não cobre apenas os circuitos $C_{1}, C_{2}$ e possivelmente alguns circuitos que passam por alguns dos arcos $x y, y x, y z, z y$. Assim, tanto $F_{i}^{\prime \prime} \cup\{x y, z y\}$ quanto $F_{i}^{\prime \prime} \cup\{y x, y z\}$ são transversais de $D$. Além disso, se por exemplo $z x \notin F_{i}^{\prime \prime}$, então $F_{i}^{\prime \prime} \cup\{x y, y a\}$ é também uma transversal de $D$. Agora mostraremos como obter a partir de $F_{1}^{\prime}, \ldots, F_{k}^{\prime}$ uma coleção $l$-disjunta $F_{1}, \ldots, F_{k}$ de transversais de $D$ usando esse tipo de extensão. Por simetria, precisamos analisar apenas dois subcasos. 


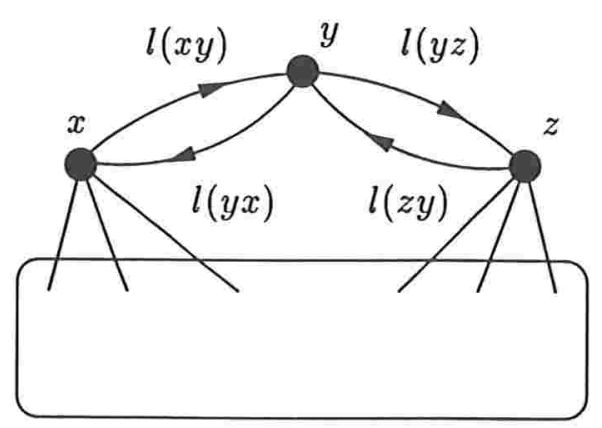

$D$



$D^{\prime}$

Figura 6.3: Caso 2 da prova do Teorema 6.3.1.

Caso 2a. $l(x y) \geq l(z y)$ e $l(y z) \leq l(y x)$.

Seja $t:=l(z y)$. Para $i=1, \ldots, t$ defina $F_{i}:=\left(F_{i}^{\prime}-\{x z, z x\}\right) \cup\{x y, z y\}$. Para $i=t+1, \ldots, k$ defina $F_{i}:=\left(F_{i}^{\prime}-\{x z, z x\}\right) \cup\{y x, y z\}$. Claramente, $F_{1}, \ldots, F_{k}$ formam uma coleção $l$-disjunta pois $l(z y)+l(y z) \geq k, l(y z) \leq l(y x)$ e $l(z y) \leq l(x y)$.

Caso 2b. $l(x y) \geq l(z y)$ e $l(y z) \geq l(y x)$.

Suponha sem perda de generalidade que $z x \in F_{i}^{\prime}$ para $i=1, \ldots, q$ e $z x \notin F_{i}^{\prime}$ para $i=q+1, \ldots, k$. Note que $q \leq l(z x)=l(z y)+l(x y)$. Além disso, temos que $l(z y)+l(y x) \leq$ $k$, pois em caso contrário, ou $z y$ ou $y x$ não está contido em um circuito mínimo, o que contraria o fato de $(D, l)$ ser crítico. Portanto, podemos supor sem perda de generalidade que $q=l(z y)+l(y x)$.

Seja $t:=l(y x)$. Para $i=1, \ldots, t$ defina $F_{i}:=\left(F_{i}^{\prime}-\{x z, z x\}\right) \cup\{y x, y z\}$. Para $i=t+1, \ldots, q$ defina $F_{i}:=\left(F_{i}^{\prime}-\{x z, z x\}\right) \cup\{z y, x y\}$. Claramente, $F_{1}, \ldots, F_{q}$ é uma coleção $l$-disjunta de $q$ transversais.

Resta-nos definir as outras $k-q$ transversais. Note que não podemos usar os arcos $y x, z y$ de novo, se quisermos obter uma coleção $l$-disjunta. Por outro lado, como $z x$ não pertence a nenhum $F_{i}^{\prime}$ para $q+1 \leq i \leq k$, podemos definir $F_{i}:=\left(F_{i}^{\prime}-\{x z\}\right) \cup\{x y, y z\}$ para $i=q+1, \ldots, k$. Como observamos acima, cada $F_{i}$ é uma transversal de $D$. Para ver que essa coleção é $l$-disjunta, só precisamos verificar se $l(x y)-l(z y) \geq k-q$ e $l(y z)-l(y x) \geq k-q$. De fato, como $k$ é a cintura de $(D, l)$, temos $k \leq l(x y)+l(y x)$ e portanto, $l(x y) \geq k-l(y x)$. Subtraindo $l(z y)$ de ambos os lados da última desigualdade e usando o fato de que $q=l(z y)+l(y x)$, temos que

$$
l(x y)-l(z y) \geq k-l(y x)-l(z y)=k-q .
$$

Analogamente, temos que $l(y z)-l(y x) \geq k-q$.

Em ambos os casos concluímos que $\operatorname{Trans}(D, l) \geq k=\operatorname{Cint}(D, l)$. Isto completa a prova. 


\section{Considerações finais}

Ao chegarmos ao final deste trabalho gostaríamos de tecer alguns comentários sobre a pesquisa realizada. A Tabela 7.1 apresenta um resumo dos resultados que obtivemos e dos que eram previamente conhecidos sobre os problemas tratados neste trabalho. Esperamos que ela seja útil àqueles que se interessam ou que venham a se interessar por essa área. Ela não indica, entretanto, os problemas que receberam maior atenção de nossa parte nem os que poderão fazer parte de uma pesquisa futura.

Um dos problemas que nos interessa sobremaneira é o de encontrar um algoritmo polinomial para o PCEC em grafos livres de Petersen. Na fase final de nossa pesquisa dedicamos uma atenção especial a ele. A grande dificuldade deste problema parece estar relacionada com o fato de que em todos os casos particulares do PCEC não-orientado para os quais se conhece um algoritmo polinomial, estes são baseados em algoritmos para encontrar um 4-fluxo ou uma 3-aresta-coloração de um grafo. Em agosto de 1999, C.Q. Zhang chamou a nossa atenção para um resultado estrutural que poderia ser útil para atacar o problema [49]. Isso deu um novo impulso em nossa pesquisa, mas até o momento em que encerramos este trabalho nenhum progresso substancial foi feito.

Não estudamos aqui problemas de cobertura uniforme por circuitos, exata ou fracionária, em grafos mistos. Certamente, estes são problemas difíceis por estarem relacionados com a conjectura da cobertura dupla por circuitos. É possível, entretanto, que alguns desses problemas possam ser resolvidos mais facilmente para classes especiais de grafos mistos. Talvez eles venham a fazer parte de nossa pesquisa no futuro.

Por fim, um problema de interesse é tentar desenvolver algoritmos de aproximação para o PCMC para grafos mistos ou classes especiais desses. Como vimos, o único resultado conhecido nessa linha é um algoritmo de 5/3-aproximação para grafos nãoorientados. Acreditamos que seja possível obter alguns resultados interessantes sobre esse problema. 


\begin{tabular}{|c|c|c|c|c|}
\hline Problema & & Grafo & Complexidade & Referência \\
\hline \multirow{9}{*}{ PCEC } & \multicolumn{2}{|l|}{ Orientado } & Polinomial & {$[42]$} \\
\hline & \multirow{4}{*}{ Não-orientado } & $\begin{array}{c}\text { 4-aresta-conexo } \\
p(e)=1 \text { ou } 2\end{array}$ & Polinomial & {$[45,44]$} \\
\hline & & Planar & Polinomial & {$[65]$} \\
\hline & & Livre de Petersen & $\begin{array}{c}\text { Polinomial } \\
\text { (sabe-se resolver apenas } \\
\text { o problema de decisão) }\end{array}$ & {$[2]$} \\
\hline & & Arbitrário & Em aberto & - \\
\hline & \multirow{4}{*}{ Misto } & Série-Paralelo & Polinomial & Capítulo 2 \\
\hline & & Larg. arb. limitada & Em aberto & - \\
\hline & & Planar & NP-difícil & Capítulo 3 \\
\hline & & Arbitrário & NP-difícil & {$[10,8]$} \\
\hline \multirow{6}{*}{ PCFC } & \multicolumn{2}{|l|}{ Orientado } & Polinomial & {$[42]$} \\
\hline & \multicolumn{2}{|l|}{ Não-orientado } & Polinomial & {$[65,10]$} \\
\hline & \multirow{4}{*}{ Misto } & Série-Paralelo & Polinomial & Capítulo 2 \\
\hline & & Larg. arb. limitada & Polinomial & - \\
\hline & & Planar & NP-completo & Capítulo 3 \\
\hline & & Arbitrário & NP-completo & {$[10,8]$} \\
\hline \multirow{6}{*}{$\mathrm{PCN} / \mathrm{PCM}$} & \multicolumn{2}{|l|}{ Orientado } & Polinomial & {$[23,16,50]$} \\
\hline & \multicolumn{2}{|l|}{ Não-orientado } & Polinomial & {$[34,68,69,50]$} \\
\hline & \multirow{4}{*}{ Misto } & Série-Paralelo & Polinomial & Capítulo 2 \\
\hline & & Larg. arb. limitada & Polinomial & - \\
\hline & & Planar & NP-completo & Capítulo 4 \\
\hline & & Arbitrário & NP-completo & {$[9,8]$} \\
\hline \multirow{8}{*}{ PCMC } & \multicolumn{2}{|l|}{ Orientado } & Polinomial & {$[27]$} \\
\hline & \multirow{4}{*}{ Não-orientado } & 4-aresta-conexo & Polinomial & {$[44]$} \\
\hline & & Planar & Polinomial & {$[15,40,59]$} \\
\hline & & Livre de Petersen & $\begin{array}{c}\text { Polinomial } \\
\text { (sabe-se apenas calcular } \\
\text { o valor ótimo) }\end{array}$ & {$[2]$} \\
\hline & & Arbitrário & NP-difícil & {$[70]$} \\
\hline & \multirow{3}{*}{ Misto } & Larg. arb. limitada & Polinomial & Capítulo 5 \\
\hline & & Planar & NP-difícil & Capítulo 5 \\
\hline & & Arbitrário & NP-difícil & {$[70]$} \\
\hline
\end{tabular}

Tabela 7.1: Tabela com os resultados sobre o PCEC, o PCFC, o PCN, o PCM e o PCMC. 


\section{Referências Bibliográficas}

[1] R.K. Ahuja, T.L. Magnanti, e J.B. Orlin. Network Flows: Theory, Algorithms and Applications. Prentice Hall, 1993.

[2] B. Alspach, L. Goddyn, e C.Q. Zhang. Graphs with the circuit cover property. Trans. Am. Math. Soc., 344 (1994), 131-154.

[3] B. Alspach e C.Q. Zhang. Cycle covers of cubic multigraphs. Discrete Math., 111 (1993), 11-17.

[4] K. Appel e W. Haken. Every map is four colorable, Part I: Discharging. Illinois J. Math, 21 (1977), 429-490.

[5] K. Appel e W. Haken. Every map is four colorable, Part II: Reducibility. Illinois J. Math, 21 (1977), 491-567.

[6] K. Appel e W. Haken. Every map is four colorable. Contemp. Math. AMS, 98 (1989).

[7] N. Alon e M. Tarsi. Covering multigraphs by simple circuits. SIAM J. Algebraic Discrete Methods, 6 (1985), 345-350.

[8] E.M. Arkin. Complexity of Cycle and Path Problems in Graphs. PhD thesis, Stanford University, 1986.

[9] E.M. Arkin e C.H. Papadimitriou. On negative cycles in mixed graphs. Oper. Res. Lett., 4 (1985), 113-116.

[10] E.M. Arkin e C.H. Papadimitriou. On the complexity of circulations. J. Algorithms, 7 (1986), 134-145.

[11] S. Arnborg, D.G Corneil e A. Proskurowski. Complexity of finding embeddings in a $k$-tree. SIAM Journal on Algebraic and Discrete Methods, 8 (1987), 277284. 
[12] S. Arnborg, J. Lagergren e D. Seese. Easy problems for tree-decomposable graphs. Journal of Algorithms, 12 (1991), 308-340.

[13] S. Arnborg e A. Proskurowski. Linear time algorithms for NP-hard problems restricted to partial $k$-trees. Discrete App. Math., 23 (1989), 11-24.

[14] V. Batagelj e T. Pisanski. On partially directed eulerian multigraphs. Publ. de l'Inst. Math. Soc., 25 (1979), 16-24.

[15] J.C. Bermond, B. Jackson, e F. Jaeger. Shortest coverings of graphs with cycles. J. Comb. Theory Ser. B, 35 (1983), 297-308.

[16] R.E. Bellman. On a routing problem. Quart. Appl. Math., 16 (1958), 87-90.

[17] H.L. Bodlaender. Dynamic programming on graphs of bounded treewidth. In Proc. 15th International Colloquium on Automata, Languages and Programming, Lecture Notes in Computer Science, 317, Springer-Verlag, Berlin (1988), 631-643.

[18] H.L. Bodlaender. A linear time algorithm for finding tree decompositions of small treewidth. In Proc. 25th Annual Association for Computing Machinery symposium on Theory of Computing, ACM Press, New York (1993), 226-234.

[19] F. Boesch e R. Tindell. Robbin's theorem for mixed multigraphs. Am. Math. Monthly, 87 (1980), 716-719.

[20] J.A. Bondy e U.S.R. Murty. Graph Theory with Applications. MacMillan Press, 1976.

[21] B. Courcelle. The monadic second order logic of graphs. I. Recognizable sets of finite graphs. Information and Computation, 85 (1990), 12-75.

[22] R. Diestel. Graph Theory. Graduate Texts in Mathematics, 173, SpringerVerlag, 1997.

[23] E.W. Dijkstra. A note on two problems in connexion with graphs. Numerische Mathematik, 1 (1959), 269-271.

[24] J. Edmonds. The Chinese postman problem. Operations Research, 13 (1965), B73-B77.

[25] J. Edmonds. Maximum matchings and a polyhedron with $(0,1)$-vertices. J. Res. Nat. Bur. Standards B, 69 (1965), 125-130.

[26] J. Edmonds e R. Giles. A min-max relation for submodular functions on graphs. Annals of Discrete Math., 1 (1977), 185-204.

[27] J. Edmonds e E.L. Johnson. Matching, Euler tours and the Chinese postman problem. Math. Programming, 5 (1973), 88-124. 
[28] M.N. Ellingham. Petersen subdivisions in some regular graphs. Congr. Numer., 44 (1984), 33-40.

[29] L. Euler. Solutio problematis and geometriam situs pertinentis. Commentarii Academiae Petropolitanae, 8 (1736), 128-140.

[30] G. Fan. Covering graphs by cycles. SIAM J. Discrete Math., 5 (1992), 491-496.

[31] H. Fleischner. Eine gemeinsame Basis für die Theorie der eulerschen Graphen und den Satz von Petersen. Monatsh. Math., 81 (1976), 267-278.

[32] H. Fleischner. Eulerian graphs and related topics, Part 1, Vol. 1. Ann. Discrete Math. , 45 (1990), North-Holland.

[33] P. Feofiloff e D.H. Younger. Directed cut transversals packing for source-sink connected graphs. Combinatorica, 7 (1987), 255-263.

[34] L.R. Ford Jr.. Network Flow Theory. The Rand Corp., P-923, 1956.

[35] L.R. Ford e D.R. Fulkerson. Flows in Networks. Princeton U. Press, 1973.

[36] A. Galluccio e M. Loebl. (P,Q)-odd digraphs. J. Graph Theory, 23 (1996), 175-184.

[37] M.R. Garey e D.S. Johnson. Computers and Intractability: A Guide to the Theory of NP-completeness. Freeman, San Francisco, 1979.

[38] L.A. Goddyn. Cones, lattices and Hilbert bases of circuits and perfect matchings. Graph structure theory (Seattle, WA, 1991), 419-439, Contemp. Math., 147, Amer. Math. Soc., Providence, RI, 1993.

[39] M. Grötschel, L. Lovász e A. Schrijver. Geometric Algorithms and Combinatorial Optimization. Springer-Verlag, 1988.

[40] M.G. Guan e H. Fleischner. On the minimum weighted cycle covering problem for planar graphs. Ars Combin., 20 (1985), 61-68.

[41] I. Holyer. The NP-completeness of edge-coloring. SIAM J. Comput., 10 (1981), 718-720.

[42] A.J. Hoffman. Some recent applications of the theory of linear inequalities to extremal combinatorial analysis. In Proc. Symp. Appl. Math, 10 (1960), 113127.

[43] W.L. Hsu. Recognizing planar perfect graphs. J. Assoc. Comput. Mach., 34 (1987), 255-288.

[44] B. Jackson. Shortest circuit covers and postman tours in graphs with a nowhere zero 4-flow. SIAM J. Comput., 19 (1990), 659-665. 
[45] F. Jaeger. Flows and generalized coloring theorems in graphs. J. Comb. Theory Ser. B, 26 (1979), 205-216.

[46] U. Jamshy e M. Tarsi. Shortest cycle covers and the cycle double cover conjecture. J. Comb. Theory Ser. B, 56 (1992), 197-204.

[47] T.R. Jensen e B. Toft. Graph Coloring Problems. Wiley, 1995.

[48] L.G. Khachiyan. A polynomial algorithm in linear programming. (Em russo) Dokl. Akad. Nauk SSSR, 244 (1979), 1093-1096. (Tradução inglesa) Soviet Mathematics Doklady, 20 (1979), 191-194.

[49] H.J. Lai e C.Q. Zhang. Hamilton weight and Petersen minor. A aparecer em J. Graph Theory.

[50] E.L. Lawler. Combinatorial Optimization: Networks and Matroids. Holt, Rinehart \& Winston, N. York, 1976.

[51] O. Lee. Passeios e Conexidade em Grafos Mistos: Algoritmos e Complexidade Computacional. Dissertação de mestrado, Instituto de Matemática e Estatística - Universidade de São Paulo, 1994.

[52] O. Lee e Y. Wakabayashi. Circuit covers in series-parallel mixed graphs. Proc. of the Third Latin American Symposium on Theoretical Informatics (LATIN) Campinas (1998) - Lecture Notes in Computer Science, 1380 (1998), Springer Verlag, 226-238.

[53] D. Lichtenstein. Planar formulae and their uses. SIAM J. Comput., 11 (1982), 329-343.

[54] C.H. Papadimitriou. On the complexity of edge traversing. Journal of ACM, 23 (1976), 544-554.

[55] O. Porto. Even induced cycles in planar graphs. Proc. of the First Latin American Symposium on Theoretical Informatics (LATIN) - São Paulo (1992) - Lecture Notes in Comput. Sci., 583 (1992), Springer Verlag, 417-429.

[56] B. Reed. Tree width and tangles: a new connectivity measure and some applications. Surveys in combinatorics, 1997 (London), 87-162, London Math. Soc. Lecture Notes Ser., 241, Cambridge Univ. Press. Cambridge, 1997.

[57] N. Robertson e P. Seymour. Graph Minors. XX. Wagner's conjecture, manuscript, 1988. Observação: a demonstração completa do teorema de Robertson e Seymour vem aparecendo numa série de artigos sob o título de Graph Minors no periódico J. Comb. Theory Ser. B, desde 1983.

[58] N. Robertson e P. Seymour. Graph Minors. II. Algorithmic aspects of treewidth. J. of Algorithms, 7 (1986), 309-322. 
[59] N. Robertson, D. Sanders, P.D. Seymour e R. Thomas. The four-colour theorem. J. Comb. Theory Ser. B, 70 (1997), 2-44.

[60] N. Robertson, P.D. Seymour e R. Thomas. Tutte's edge-coloring conjecture. J. Comb. Theory Ser. B, 70 (1997), 166-183.

[61] A. Schrijver. A counterexample to a conjecture of Edmonds and Giles. Discrete Math., 32 (1980), 213-214.

[62] A. Schrijver. A min-max relation for directed graphs. Annals of Discrete Math., 16 (1982), 261-280.

[63] A. Schrijver. Theory of Linear and Integer Programming. Wiley, 1986.

[64] A. Sebő. Hilbert bases, Carathéodory's theorem and combinatorial optimization. In R. Kannan and W.R. Pulleyblank, editors, Integer Programming and Combinatorial Optimization Proceedings, 431-456, Waterloo, University of Waterloo Press, 1990.

[65] P.D. Seymour. Sum of circuits. Em Graph Theory and Related Topics, 341-355. Academic Press, N. York, 1979.

[66] P.D. Seymour. On multi-coloring of cubic graphs and the conjecture of Fulkerson and Tutte. Proc. London Math. Soc., 38 (1979), 423-460.

[67] G. Szekeres. Polyhedral decompositions of cubic graphs. Bull. Austral. Math. Soc., 8 (1973), 367-387.

[68] R.L. Tobin. Finding a minimal weight path in an undirected network, Em 44th National ORSA Meeting, San Diego, California (1973).

[69] R.L. Tobin. Minimal complete matchings and negative cycles. Networks, 5 (1975), 371-387.

[70] C. Thomassen. On the complexity of finding a minimum cycle cover of a graph. SIAM J. Comput., 26 (1997), 675-677.

[71] W.T. Tutte. On the algebraic theory of graph colourings. J. Comb. Theory, 1 (1966), 15-50.

[72] W.T. Tutte. A geometrical version of the four color problem. Combinatorial Mathematics and its Applications, (eds. R.C. Bose e T.A. Dowling), University of North Carolina Press, Chapel Hill, 1967.

[73] D.R. Woodall. Menger and König systems. Theory and applications of graphs (Proc. Intern. Conf., Western Mich. Univ.), Lecture Notes in Math., 642 (1978), 620-635. 
[74] C.Q. Zhang. Integer Flows and Cycle Covers of Graphs. Monographs and Textbooks in Pure and Applied Mathematics, 205, Marcel Dekker, Inc., New York, 1997.

[75] C.Q. Zhang. Minimum cycle coverings and integer flows. J. Graph Theory, 14 (1990), 537-546. 


\title{
Índice Remissivo
}

\author{
$A(M), 2$ \\ $D=(V, A), 2$ \\ $E(M), 2$ \\ $G(M), 2$ \\ $G=(V, E), 2$ \\ $M-F, 3$ \\ $M / F, 3$ \\ $M / e, 3$ \\ $M=D \sqcup G, 2$ \\ $V(M), 2$ \\ $\delta_{D}^{+}(X), 2$ \\ $\delta_{D}^{-}(X), 2$ \\ $\delta_{G}(X), 2$ \\ $\delta_{G}(X, Y), 2$ \\ $\nabla_{M}(X), 2$ \\ arcos, 1 \\ paralelos, 2 \\ reversos, 2 \\ arestas, 1 \\ paralelas, 2 \\ caminho, 3 \\ extremos do, 3 \\ de um elemento, 3 \\ corte, 2 \\ aresta de, 2 \\ dicorte, 2 \\ extremos, 1 \\ grafo \\ composto, 2 \\ euleriano, 3 \\ fortemente conexo, 3 \\ misto, 1 \\ não-orientado, 2 \\ orientado, 2 \\ série-paralelo, 3 \\ subjacente, 2 \\ junção, 78 \\ laço, 2 \\ livre, 3 \\ menor, 3 \\ parte \\ ciclo, 4 \\ vértices internos, 3 \\ ciclo orientado, 4 \\ circuito, 3 \\ circuitos \\ matriz de incidência dos, 6 \\ coleção $l$-disjunta, 78 \\ componente fortemente conexo, 3 \\ cone, 4 \\ recessional, 4 \\ contração \\ de um conjunto, 3 \\ não-orientada, 2 \\ orientada, 2 \\ passeio, 3 \\ comprimento do, 3 \\ fechado, 3 \\ final do, 3 \\ início do, 3 \\ poliedro, 4 \\ bem-descrito, 5 \\ ponte, 2 \\ problema da separação, 5
}


remoção, 3

subdivisão de aresta, 3

subdivisão de um grafo, 3

tamanho da codificação, 4

transversal, 78

vértices, 1 